\title{
Formation of Galaxy Clusters
}

\author{
Andrey V. Kravtsov ${ }^{1}$ and Stefano Borgani ${ }^{2}$ \\ ${ }^{1}$ Department of Astronomy $\mathcal{E}$ Astrophysics, Kavli Institute for Cosmological Physics, The \\ University of Chicago, Chicago, IL 60637 \\ email: andrey@oddjob.uchicago.edu \\ ${ }^{2}$ Dipartimento di Fisica dellUniversità di Trieste, Sezione di Astronomia, I-34131 Trieste, Italy; \\ INAF Osservatorio Astronomico di Trieste, Italy; \\ and INFN Istituto Nazionale di Fisica Nucleare, Trieste, Italy; \\ email: borgani@oats.inaf.it
}

\begin{abstract}
Formation of galaxy clusters corresponds to the collapse of the largest gravitationally bound overdensities in the initial density field and is accompanied by the most energetic phenomena since the Big Bang and by the complex interplay between gravity-induced dynamics of collapse and baryonic processes associated with galaxy formation. Galaxy clusters are, thus, at the cross-roads of cosmology and astrophysics and are unique laboratories for testing models of gravitational structure formation, galaxy evolution, thermodynamics of the intergalactic medium, and plasma physics. At the same time, their large masses make them a useful probe of growth of structure over cosmological time, thus providing cosmological constraints that are complementary to other probes. In this review, we describe our current understanding of cluster formation: from the general picture of collapse from initial density fluctuations in an expanding Universe to detailed simulations of cluster formation including the effects of galaxy formation. We outline both the areas in which highly accurate predictions of theoretical models can be obtained and areas where predictions are uncertain due to uncertain physics of galaxy formation and feedback. The former includes the description of the structural properties of the dark matter halos hosting cluster, their mass function and clustering properties. Their study provides a foundation for cosmological applications of clusters and for testing the fundamental assumptions of the standard model of structure formation. The latter includes the description of the total gas and stellar fractions, the thermodynamical and non-thermal processes in the intracluster plasma. Their study serves as a testing ground for galaxy formation models and plasma physics. In this context, we identify a suitable radial range where the observed thermal properties of the intra-cluster plasma exhibit the most regular behavior and thus can be used to define robust observational proxies for the total cluster mass. Finally, we discuss the formation of clusters in non-standard cosmological models, such as non-Gaussian models for the initial density field and models with modified gravity, along with prospects for testing these alternative scenarios with large cluster surveys in the near future.
\end{abstract}

Key words: Cosmology, galaxy clusters, intra-cluster medium 


\section{Contents}

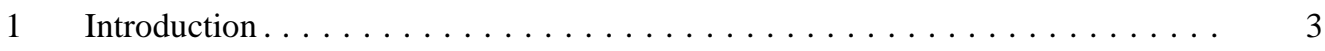

2 The observed properties of galaxy clusters $\ldots \ldots \ldots \ldots \ldots \ldots \ldots \ldots$

3 Understanding the formation of galaxy clusters $\ldots \ldots \ldots \ldots \ldots \ldots \ldots$

3.1 Initial density perturbation field and its linear evolution $\ldots \ldots \ldots \ldots \ldots \ldots$

3.2 Non-linear evolution of spherical perturbations and non-linear mass scale .... 12

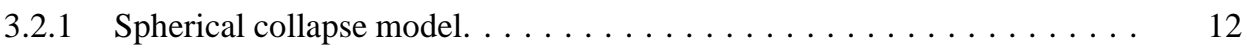

3.2.2 The nonlinear mass scale $M_{\mathrm{NL}} \ldots \ldots \ldots \ldots \ldots \ldots \ldots \ldots \ldots$

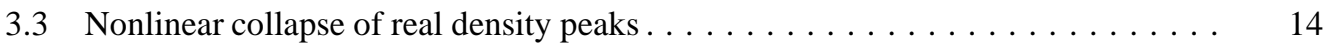

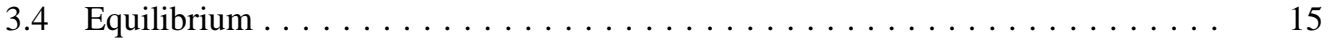

3.5 Internal structure of cluster halos $\ldots \ldots \ldots \ldots \ldots \ldots \ldots \ldots \ldots \ldots \ldots$

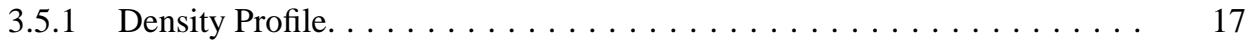

3.5.2 Velocity dispersion profile and velocity anisotropy.......... 20

3.5.3 Shape. ........................ 1

3.6 Mass definitions . . . . . . . . . . 2

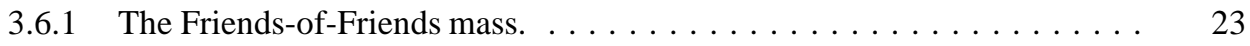

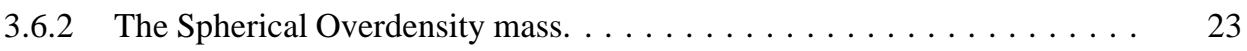

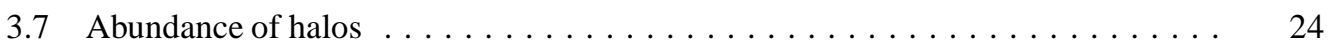

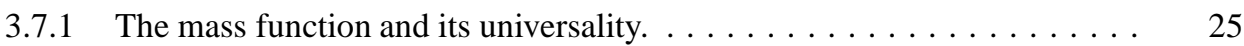

3.7.2 Calibrations of halo mass function in cosmological simulations....... 27

3.8 Clustering of halos $\ldots \ldots \ldots \ldots \ldots \ldots \ldots \ldots \ldots \ldots \ldots \ldots \ldots \ldots \ldots \ldots \ldots$

3.9 Self-similar evolution of galaxy clusters $\ldots \ldots \ldots \ldots \ldots \ldots \ldots \ldots \ldots$

3.9.1 Self-similar model: assumptions and basic expectations. . . . . . . . 30

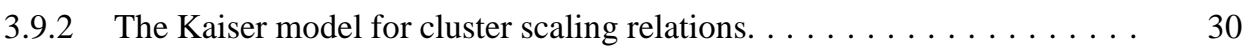

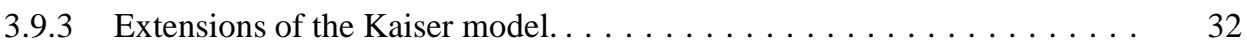

3.9.4 Practical implications for observational calibrations of scaling relations. . 34

3.10 Cluster formation and Thermodynamics of the Intra-cluster gas . . . . . . . . . 35

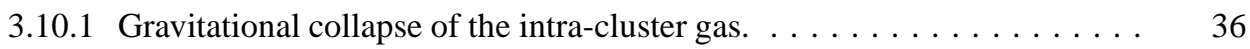

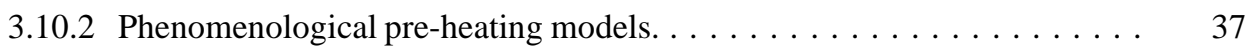

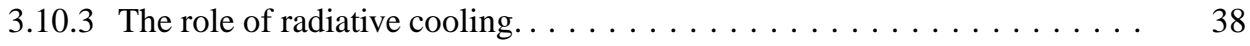

3.10.4 Thermodynamics of the intracluster medium with stellar and active galactic

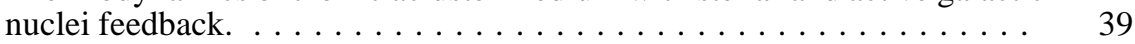

4 Regularity of the cluster populations $\ldots \ldots \ldots \ldots \ldots \ldots \ldots \ldots \ldots \ldots$

4.1 Characterizing regularity $\ldots \ldots \ldots \ldots \ldots \ldots \ldots \ldots \ldots \ldots \ldots \ldots \ldots \ldots$

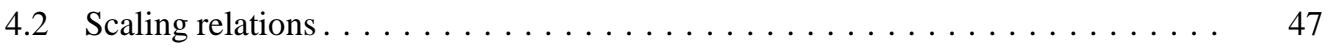

5 Cluster formation in alternative cosmological models . . . . . . . . . . . 50

5.1 Mass function and bias of clusters in non-Gaussian models . . . . . . . . . . . 51

5.2 Formation of clusters in modified gravity models $\ldots \ldots \ldots \ldots \ldots \ldots$

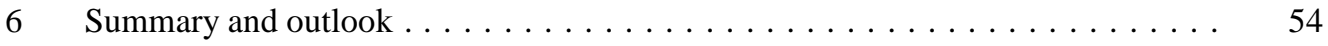




\section{Introduction}

Tendency of nebulae to cluster has been ddiscovered by Charles Messier and William Herschel, who have constructed the first systematic catalogs of these objects. This tendency has become more apparent as larger and larger samples of galaxies were compiled in the 19th and early 20th centuries. Studies of the most prominent concentrations of nebulae, the clusters of galaxies, were revolutionized in the 1920s by Edwin Hubble's proof that spiral and elliptical nebulae were bona fide galaxies like the Milky Way located at large distances from us (Hubble 1925, 1926), which implied that clusters of galaxies are systems of enormous size. Just a few years later, measurements of galaxy velocities in regions of clusters made by Hubble \& Humason (1931) and assumption of the virial equilibrium of galaxy motions were used to show that the total gravitating cluster masses for the Coma (Zwicky 1933, and see also Zwicky 1937) and Virgo clusters (Smith 1936) were enormous as well.

The masses implied by the measured velocity dispersions were found to exceed combined mass of all the stars in clusters galaxies by factors of $\sim 200-400$, which prompted Zwicky to postulate the existence of large amounts of "dark matter" (DM), inventing this widely used term in the process. Although the evidence for dark matter in clusters was disputed in the subsequent decades, as it was realized that stellar masses of galaxies were underestimated in the early studies, dark matter was ultimately confirmed by the discovery of extended hot intracluster medium (ICM) emitting at X-ray energies by thermal bremsstrahlung that was found to be smoothly filling intergalactic space within the Coma cluster (Cavaliere, Gursky \& Tucker 1971; Forman et al. 1972; Gursky et al. 1971; Kellogg et al. 1972; Meekins et al. 1971). The X-ray emission of the ICM has not only provided a part of the missing mass (as was conjectured on theoretical grounds by Limber 1959, van Albada 1960), but also allows the detection of clusters out to $\mathrm{z}>1$ Rosati, Borgani \& Norman (2002). Furthermore, measurement of the ICM temperature has provided an independent confirmation that the depth of gravitational potential of clusters requires additional dark component. It was also quickly realized that inverse Compton scattering of the cosmic microwave background (CMB) photons off thermal electrons of the hot intergalactic plasma should lead to distortions in the CMB spectrum, equivalent to black body temperature variations of about $10^{-4}-10^{-5}$ [the Sunyaev-Zel'dovich (SZ) effect;Sunyaev \& Zeldovich 1980, 1970, 1972b. This effect has now been measured in hundreds of clusters (e.g., Carlstrom, Holder \& Reese 2002).

Given such remarkable properties, it is no surprise that the quest to understand the formation and evolution of galaxy clusters has become one of the central efforts in modern astrophysics over the past several decades. Early pioneering models of collapse of initial density fluctuations in the expanding Universe have shown that systems resembling the Coma cluster can indeed form (Peebles 1970, van Albada 1960, 1961, White 1976). Gott \& Gunn (1971, see also Sunyaev \& Zeldovich 1972a) showed that hot gas observed in the Coma via X-ray observations can be explained within such a collapse scenario by heating of the infalling gas by the strong accretion shocks. Subsequently, emergence of the hierarchical model of structure formation (Gott \& Rees 1975, Press \& Schechter 1974, White \& Rees 1978), combined with the cold dark matter (CDM) cosmological scenario (Blumenthal et al. 1984; Bond, Szalay \& Turner 1982), provided a powerful framework for interpretation of the multi-wavelength cluster observations. At the same time, rapid advances in computing power and new, efficient numerical algorithms have allowed fully three-dimensional $a b$ initio numerical calculations of cluster formation within self-consistent cosmological context in both dissipationless regime (Efstathiou et al. 1985, Klypin \& Shandarin 1983) and 
including dissipational baryonic component (Evrard 1988, 1990).

In the past two decades, theoretical studies of cluster formation have blossomed into a vibrant and mature scientific field. As we detail in the subsequent sections, the standard scenario of cluster formation has emerged and theoretical studies have identified the most important processes that shape the observed properties of clusters and their evolution, which has enabled usage of clusters as powerful cosmological probes (see, e.g., Allen, Evrard \& Mantz 2011, for a recent review). At the same time, observations of clusters at different redshifts have highlighted several key discrepancies between models and observations, which are particularly salient in the central regions (cores) of clusters.

In the current paradigm of structure formation clusters are thought to form via an hierarchical sequence of mergers and accretion of smaller systems driven by gravity and DM that dominates the gravitational field. Theoretical models of clusters employ a variety of techniques determined by a particular aspect of cluster formation they aim to understand. Many of the bulk properties of clusters are thought to be determined solely by the initial conditions, dissipationless DM that dominates cluster mass budget, and gravity. Thus, cluster formation is often approximated in models as DM-driven dissipationless collapse from cosmological initial conditions in an expanding Universe. Such models are quite successful in predicting the existence and functional form of correlations between cluster properties, as well as their abundance and clustering, as we discuss in detail in Section 3. One of the most remarkable models of this kind is a simple self-similar model of clusters (Kaiser 1986, see $\$ 3.9$ below). Despite its simplicity, the predictions of this model are quite close to results of observations and have, in fact, been quite useful in providing baseline expectations for evolution of cluster scaling relations. Studies of abundance and spatial distribution of clusters using dissipationless cosmological simulations show that these statistics retain remarkable memory of the initial conditions.

The full description of cluster formation requires detailed modeling of the non-linear processes of collapse and the dissipative physics of baryons. The gas is heated to high, X-ray emitting temperatures by adiabatic compression and shocks during collapse and settles in hydrostatic equilibrium within the cluster potential well. Once the gas is sufficiently dense, it cools, the process that can feed both star formation and accretion onto supermassive black holes (SMBHs) harbored by the massive cluster galaxies. The process of cooling and formation of stars and SMBHs can then result in energetic feedback due to supernovae $(\mathrm{SNe})$ or active galactic nuclei (AGN), which can inject substantial amounts of heat into the ICM and spread heavy elements throughout the cluster volume.

Galaxy clusters are therefore veritable crossroads of astrophysics and cosmology: While abundance and spatial distribution of clusters bear indelible imprints of the background cosmology, gravity law, and initial conditions, the nearly closed-box nature of deep cluster potentials makes them ideal laboratories to study processes operating during galaxy formation and their effects on the surrounding intergalactic medium.

In this review we discuss the main developments and results in the quest to understand the formation and evolution of galaxy clusters. Given the limited space available for this review and the vast amount of literature and research directions related to galaxy clusters, we have no choice but to limit the focus of our review, as well as the number of cited studies. Specifically, we focus on the most basic and well-established elements of the standard paradigm of DM-driven hierarchical structure formation within the framework of $\Lambda$ CDM cosmology as it pertains to galaxy clusters. We focus mainly on the theoretical predictions of the properties of the total cluster mass distribution and properties of the hot intracluster gas, and 
only briefly discuss results pertaining to the evolution of stellar component of clusters, understanding of which is still very much a work in progress. Comparing model predictions to real clusters, we mostly focus on comparisons with X-ray observations, which have provided the bulk of our knowledge of ICM properties so far. In $\S 5$, we briefly discuss the differences in formation of clusters in models with the non-Gaussian initial conditions and modified gravity. Specifically, we focus on the information that statistics sensitive to the cluster formation process, such as cluster abundance and clustering, can provide about the primordial non-Gaussianity and possible deviations of gravity from General Relativity. We refer readers to recent extensive reviews on cosmological uses of galaxy clusters by Allen, Evrard \& Mantz (2011) and Weinberg et al. (2012) for a more extensive discussion of this topic.

\section{The observed properties of galaxy clusters}

Observational studies of galaxy clusters have now developed into a broad, multi-faceted and multi-wavelength field. Before we embark on our overview of different theoretical aspects of cluster formation, we briefly review the main observational properties of clusters and, in particular, the basic properties of their main matter constituents.

Figure 1 shows examples of the multiwavelength observations of two massive clusters at two different cosmic epochs: the Abell 1689 at $\mathrm{z}=0.18$ and the SPT-CL J2106-5844 at $\mathrm{z}=1.133$. It illustrates all of the main components of the clusters: the luminous stars in and around galaxies (the intracluster light or ICL), the hot ICM observed via its X-ray emission and the Sunyaev-Zel'dovich effect and, in the case of Abell 1689, even the presence of invisible DM manifesting itself through gravitational lensing of background galaxies distorting their images into extended, cluster-centric arcs Bartelmann 2010, and references therein). At larger radii, the lensing effect is weaker. Although not easily visible by eye, it can still be reliably measured by averaging the shapes of many background galaxies and comparing the average with the expected value for an isotropic distribution of shapes. The gravitational lensing is a direct probe of the total mass distribution in clusters, which makes it both extremely powerful in its own right and a very useful check of other methods of measuring cluster masses. The figure shows several bright elliptical galaxies that are typically located near the cluster center. A salient feature of such central galaxies is that they show little evidence of ongoing star formation, despite their extremely large masses.

The diffuse plasma is not associated with individual galaxies and constitutes the intracluster medium, which contains the bulk of the normal baryonic matter in massive clusters. Although the hot ICM is not directly associated with galaxies, their properties are correlated. For example, Fig. 2 shows the mass of the ICM gas within the radius $R_{500}$, defined as the radius enclosing mean overdensity of $\Delta_{c}=500 \rho_{\mathrm{cr}}$, versus stellar mass in galaxies within the same radius for a number of local $(\mathrm{z} \lesssim 0.1)$ and distant $(0.1<\mathrm{z}<0.6)$ clusters (Lin et al. 2012). Here $\rho_{\mathrm{cr}}(\mathrm{z})=3 H(\mathrm{z})^{2} /(8 \pi G$ is the critical mean density of the Universe, defined in terms of the Hubble function $H(\mathrm{z})$. The figure shows a remarkably tight, albeit non-linear, correlation between these two baryonic components. It also shows that the gas mass in clusters is on average about ten times larger than the mass in stars, although this ratio is systematically larger for smaller mass clusters, ranging from $M_{*} / M_{\mathrm{g}} \approx 0.2$ to $\approx 0.05$, as mass increases from group scale $\left(M_{500} \sim\right.$ few $\left.\times 10^{13} \mathrm{M}_{\odot}\right)$ to massive clusters $\left(M_{500} \sim 10^{15} \mathrm{M}_{\odot}\right)$. 

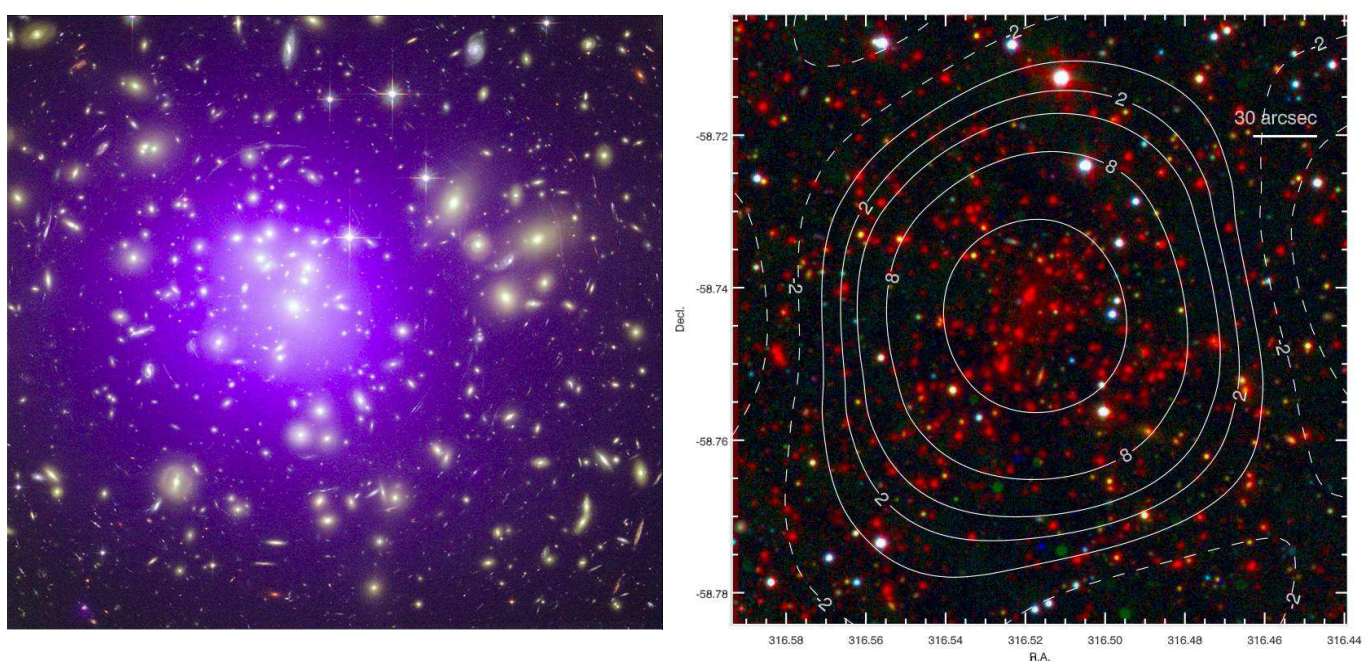

Fig. 1. Left panel: the composite X-ray/optical image (556 kpc on a side) of the galaxy cluster Abell 1689 at redshift $\mathrm{z}=0.18$. The purple haze shows $\mathrm{X}$-ray emission of the $T \sim 10^{8} \mathrm{~K}$ gas, obtained by the Chandra $X$-ray Observatory. Images of galaxies in the optical band, colored in yellow, are from observations performed with the Hubble Space Telescope. The long arcs in the optical image are caused by the gravitational lensing of background galaxies by matter in the galaxy cluster, the largest system of such arcs ever found (Credit:X-ray: NASA/CXC/MIT; Optical: NASA/STScI). Right panel: the galaxy cluster SPT-CL J2106-5844 at $\mathrm{z}=1.133$, the most massive cluster known at $\mathrm{z}>1$ discovered via its Sunyaev-Sel'dovich $(\mathrm{SZ})$ signal $\left(M_{200} \approx 1.3 \times 10^{15} \mathrm{M}_{\odot}\right)$. The color image shows the Magellan/LDSS3 optical and Spitzer/IRAC mid-infrared measurements (corresponding to the blue-green-red color channels). The frame subtends $4.8 \times 4.8$ arcmin, which corresponds to $2.4 \times 2.4 \mathrm{Mpc}$ at the redshift of the cluster. The white contours correspond to the South Pole Telescope SZ significance values, as labeled, where dashed contours are used for the negative significance values. (Adapted from Foley et al. 2011).

The temperature of the ICM is consistent with velocities of galaxies and indicates that both galaxies and gas are nearly in equilibrium within a common gravitational potential well. The mass of galaxies and hot gas is not sufficient to explain the depth of the potential well, which implies that most of the mass in clusters is in a form of DM. Given that hydrogen is by far the most abundant element in the Universe, most of the plasma particles are electrons and protons, with a smaller number of helium nuclei. There are also trace amounts of heavier nuclei some of which are only partially ionized. The typical average abundance of the heavier elements is about one-third of that found in the Sun or a fraction of one per cent by mass; it decreases with increasing radius and can be quite inhomogeneous, especially in merging systems (Werner et al. 2008, for a review).

Thermodynamic properties of the ICM are of utmost importance, because comparing such properties to predictions of baseline models without cooling and heating can help to isolate the impact of these physical processes in cluster formation. The most popular baseline model is the self-similar model of clusters developed by Kaiser (1986), which we consider in detail in Section 3.9 below. In its simplest version, this model assumes that clusters are scaled versions of each other, so that gas density at a given fraction of the characteristic 


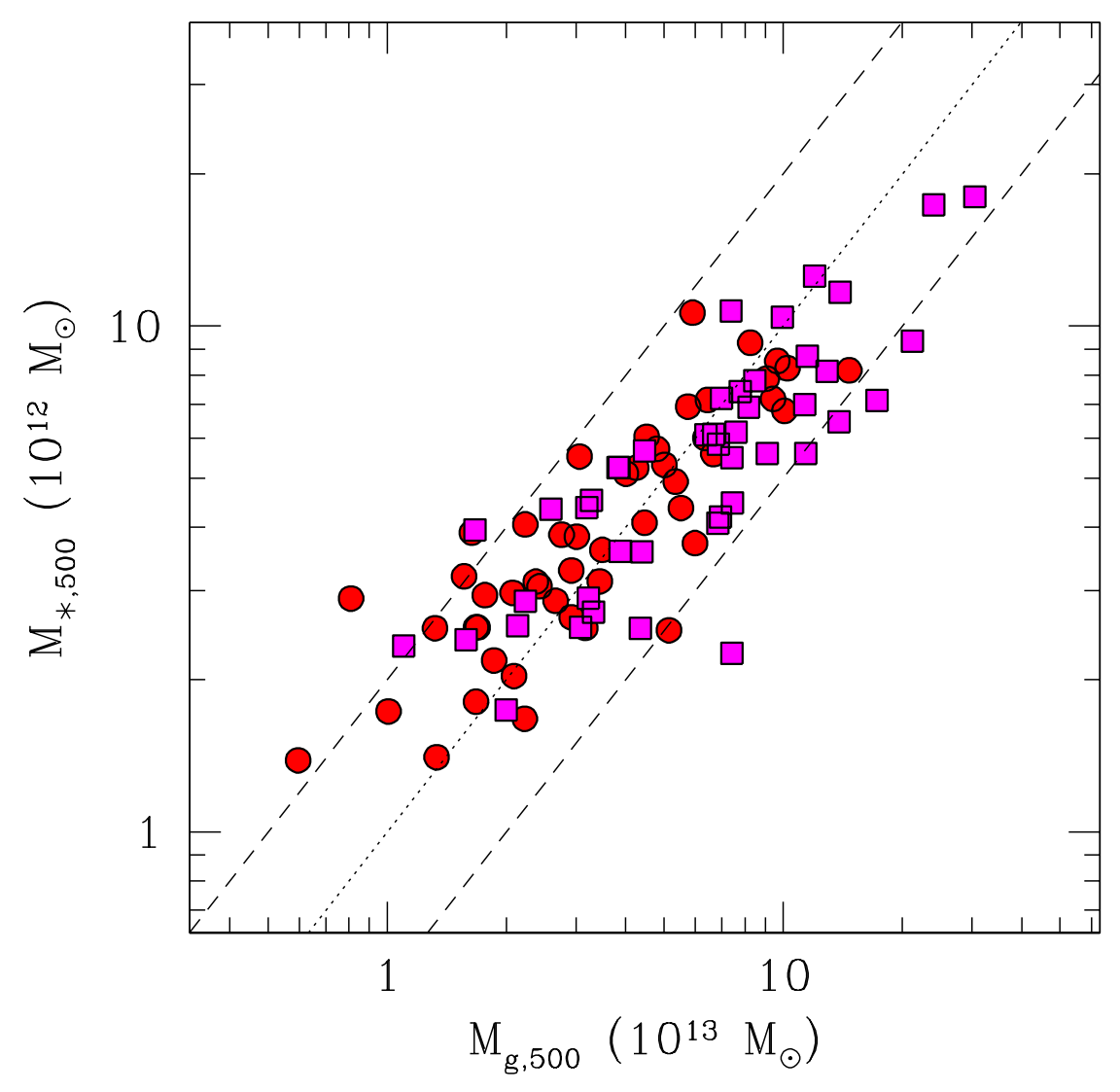

Fig. 2. The mass in stars vs. the mass of hot, X-ray emitting gas. Both masses are measured within the radius $R_{500}$ estimated from the observationally calibrated $Y_{\mathrm{X}}-M_{500}$ relation, assuming flat $\Lambda \mathrm{CDM}$ cosmology with $\Omega_{\mathrm{m}}=1-\Omega_{\Lambda}=0.26$ and $h=0.71$. Red circles show local clusters located at $\mathrm{z}<0.1$, whereas magenta squares show higher-redshift clusters: $0.1<\mathrm{z}<0.6$ (see Lin et al. 2012, for details). The dotted line corresponds to the constant stellar-to-gas mass ratio $M_{*, 500} / M_{\mathrm{g}, 500}=0.1$, whereas the dashed lines correspond to the values of 0.05 and 0.2 for this ratio.

radius of clusters, defined by their mass, is independent of cluster mass. Figure 3 shows the electron density in clusters as a function of ICM temperature (and hence mass) at different radii. It is clear that density is independent of temperature only outside cluster core at $r \sim R_{500}$, although there is an indication that density is independent of temperature at $r=R_{2500}$ for $k_{B} T \gtrsim 3 \mathrm{keV}$. This indicates that processes associated with galaxy formation and feedback affect the properties of clusters at $r \lesssim R_{2500}$, but their effects are mild at larger radii.

During the past two decades, it has been established that the core regions of the relaxed clusters are generally characterized by a strongly peaked X-ray emissivity, indicating efficient cooling of the gas (e.g., Fabian 1994). Quite interestingly, spectroscopic observations with the Chandra and XMM-Newton satellites have demonstrated that, despite strong Xray emission of the hot gas, only a relatively modest amount of this gas cools down to low temperatures (e.g., Böhringer et al. 2001, Peterson et al. 2001). This result is generally 


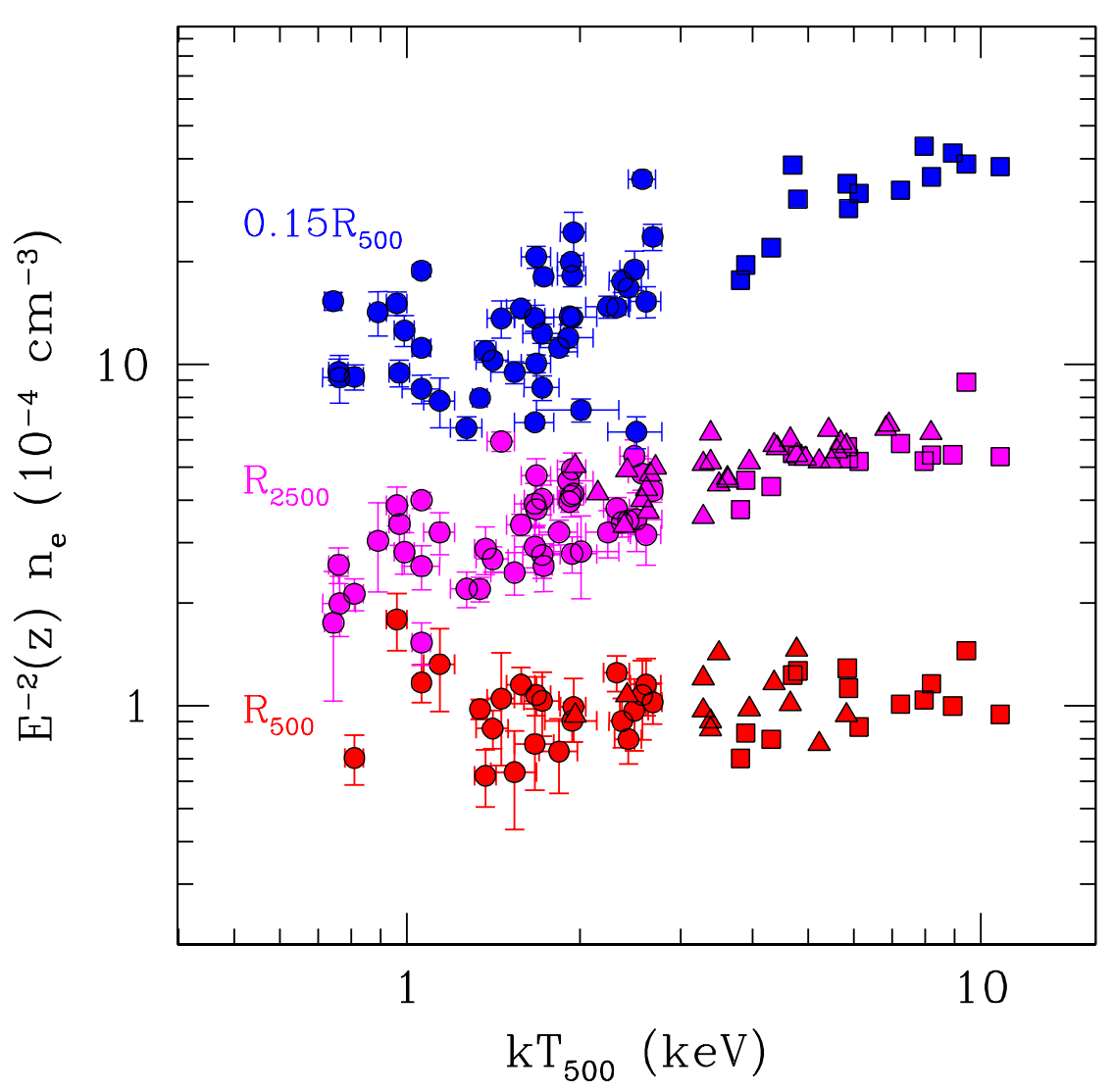

Fig. 3. The observed electron number density, $n_{e}$, in galaxy clusters and groups, measured at different radii (from top to bottom: $0.15 R_{500}, R_{2500}, R_{500}$; see labels) as a function of the intracluster medium temperature at $R_{500}$. The values of $n_{e}$ are rescaled by $E^{-2}(\mathrm{z})$, the scaling expected from the definition of the radii at which densities are measured. Squares and circles show systems observed with the Chandra X-ray Observatory from the studies by Vikhlinin et al. (2009a) and Sun et al. (2009), triangles show systems observed with the XMM-Newton telescope by Pratt et al. (2010). Note that electron densities at large radii are independent of temperature, as expected from the self-similar model, whereas at small radii the rescaled densities increase with temperature. Note also that the scatter from cluster to cluster increases with decreasing radius, especially for low-temperature groups (after Sun 2012).

consistent with the low levels of star formation observed in the brightest cluster galaxies (BCGs; e.g., McDonald et al. 2011). It implies that a heating mechanism should compensate for radiative losses, thereby preventing the gas in cluster cores to cool down to low temperature. The presence of cool cores is also reflected in the observed temperature profiles (e.g. Leccardi \& Molendi 2008, Pratt et al. 2007, Vikhlinin et al. 2006, see also Figure 4), which exhibit decline of temperature with decreasing radius in the innermost regions of relaxed cool-core clusters.

One of the most important and most widely studied aspects of ICM properties are correlations between its different observable integrated quantities and between observable 


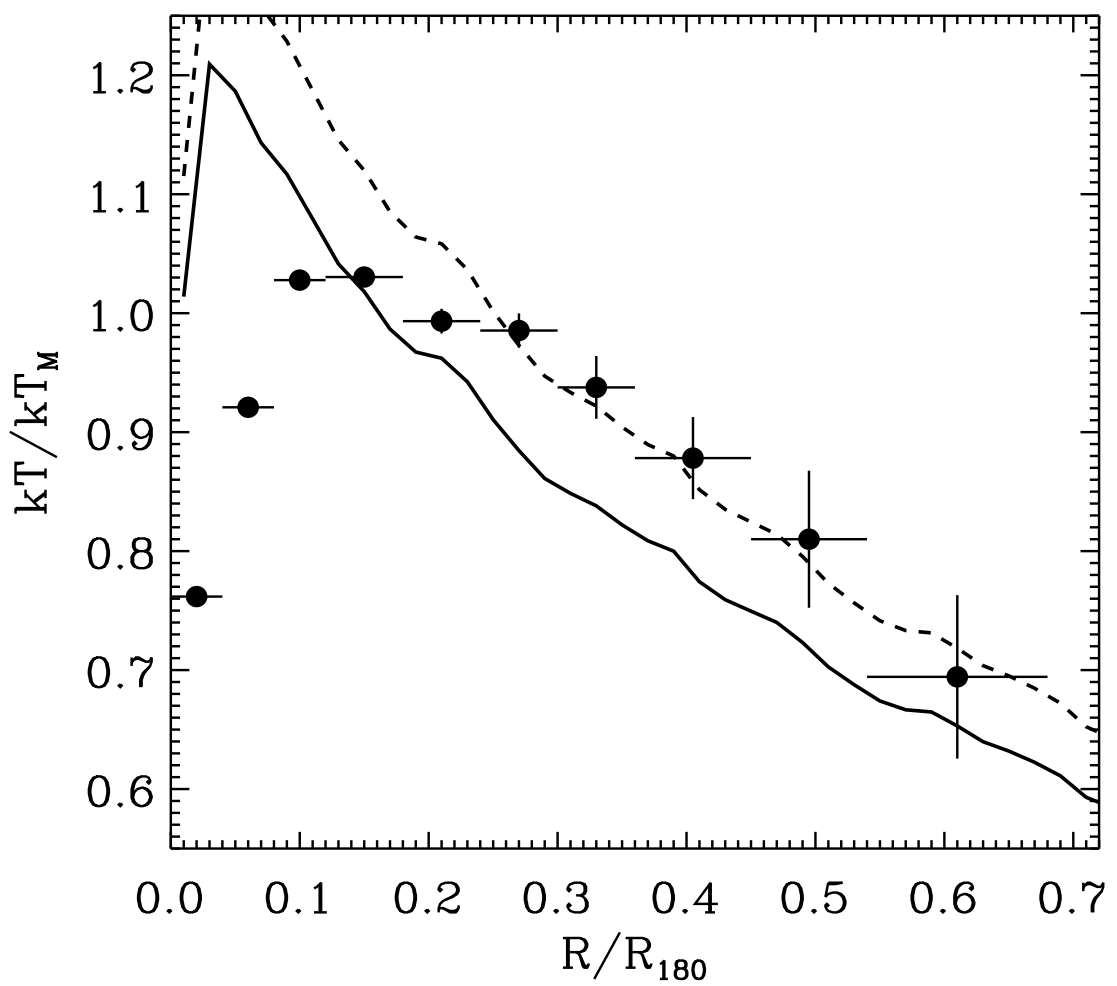

Fig. 4. Comparison between temperature profiles, normalized to the global temperature measured within $R_{180}$ by Leccardi \& Molendi (2008), for a set of about 50 nearby clusters with $\mathrm{z} \lesssim 0.3$ and with temperature $k_{B} T_{X}>3 \mathrm{keV}$, observed with the XMM-Newton (dots with errorbars) and results from cosmological hydrodynamical simulations including the effect of radiative cooling, star formation and supernova feedback in the form of galactic winds (solid curve; Borgani et al. 2004). From Leccardi \& Molendi (2008).

quantities and total mass. Such scaling relations are the key ingredient in cosmological uses of clusters, where it is particularly desirable that the relations are characterized by small scatter and are independent of the relaxation state and other properties of clusters. Although clusters are fascinatingly complex systems overall, they do exhibit some remarkable regularities. As an example, Figure 5 shows the correlation between the bolometric luminosity emitted from within $R_{500}$ and the $Y_{\mathrm{X}}$ parameter defined as a product of gas mass within $R_{500}$ and ICM temperature derived from the $\mathrm{X}$-ray spectrum within the radial range $(0.15-1) R_{500}$ Kravtsov, Vikhlinin \& Nagai 2006) for theRepresentative XMM-Newton Cluster Structure Survey (REXCESS) sample of clusters studied by Pratt et al. (2009). Different symbols indicate clusters in different states of relaxation, whereas clusters with strongly peaked central gas distribution (the cool core clusters) and clusters with less centrally concentrated gas distribution are shown with different colors. The left panel shows total luminosity integrated within radius $R_{500}$, wheareas the right panel shows luminosity calculated with the central region within $0.15 R_{500}$ excised. Quite clearly, the core-excised $\mathrm{X}$-ray luminosity exhibits remarkably tight correlation with $Y_{\mathrm{X}}$, which, in turn, is expected to correlate tightly with total cluster mass (Fabjan et al. 2011; Kravtsov, Vikhlinin \& Nagai 2006; Stanek et al. 2010). This figure illustrates the general findings in the past decade that clusters exhibit strong regularity and tight correlations among X-ray observable quantities 

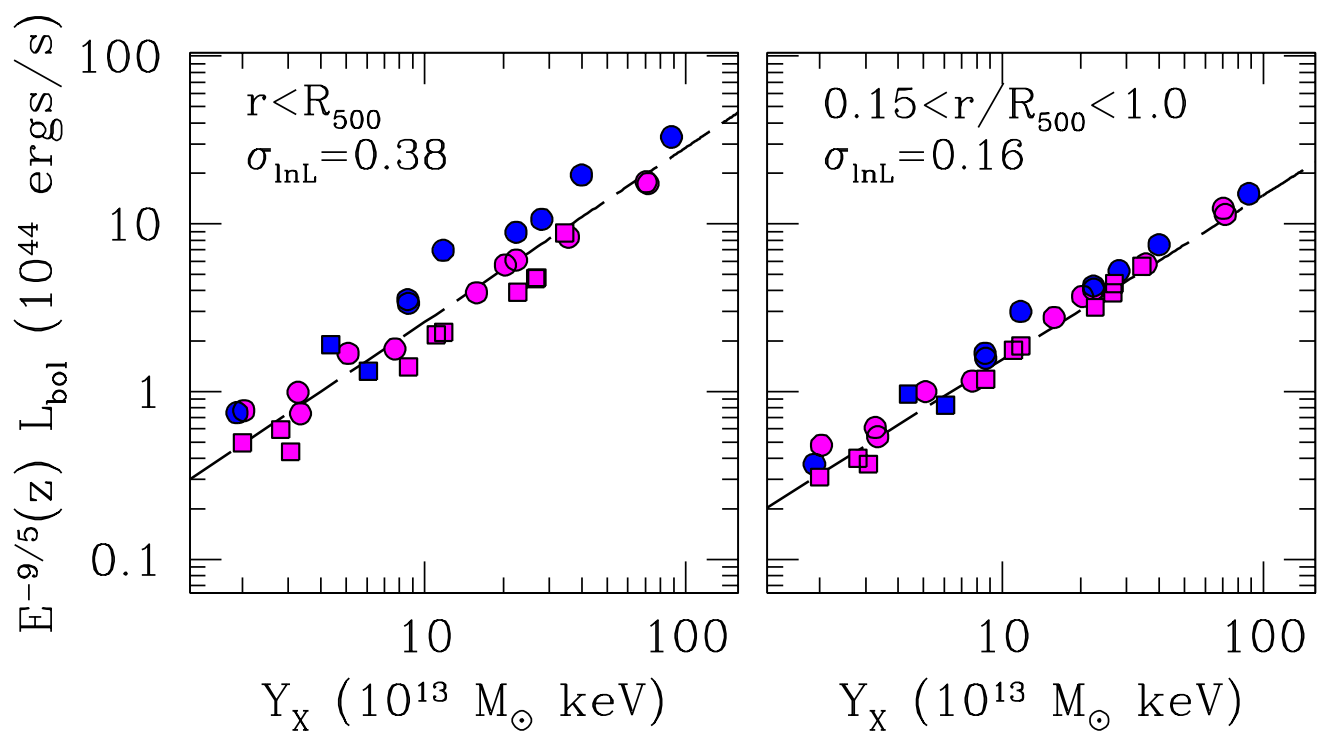

Fig. 5. Correlation of bolometric luminosity of intracluster gas and $Y_{\mathrm{X}} \equiv M_{\mathrm{gas}} T_{X}$, where $M_{\mathrm{gas}}$ is the mass of the gas within $R_{500}$ and $T_{X}$ is temperature derived from the fit to gas spectrum accounting only for emission from radial range $(0.15-1) R_{500}$. Results are shown for the local clusters from the Representative XMM-Newton Cluster Structure Survey sample of Pratt et al. (2009). The left panel shows total luminosity integrated within radius $R_{500}$, whereas the right panel shows bolometric luminosity calculated with the central $0.15 R_{500}$ of the cluster excised. Labels in the top left corner indicate the radial range used in computing the luminosity and logarithmic scatter of luminosity at fixed $Y_{\mathrm{X}}$. The blue points show cool core clusters, whereas magenta points are non-cool core clusters. Clusters classified as relaxed and disturbed are shown by circles and squares, respectively. Note that exclusion of the cluster cores reduces the scatter between luminosity and $Y_{\mathrm{X}}$ by more than a factor of two.

and total mass, provided that relevant quantities are measured after excluding the emission from cluster cores.

\section{Understanding the formation of galaxy clusters}

\subsection{Initial density perturbation field and its linear evolution}

In the currently standard hierarchical structure formation scenario, objects are thought to form via gravitational collapse of peaks in the initial primordial density field characterized by the density contrast (or overdensity) field: $\delta(\mathbf{x})=\left(\rho(\mathbf{x})-\bar{\rho}_{\mathrm{m}}\right) / \bar{\rho}_{\mathrm{m}}$, where $\bar{\rho}_{\mathrm{m}}$ is the mean mass density of the Universe. Properties of the field $\delta(\mathbf{x})$ depend on specific details of the processes occurring during the earliest inflationary stage of evolution of the Universe (Bardeen, Steinhardt \& Turner 1983; Guth \& Pi 1982; Starobinsky 1982) and the subsequent stages prior to recombination (Bardeen et al. 1986, Bond \& Efstathiou 1984, Eisenstein \& Hu 1999, Peebles 1982). A fiducial assumption of most models that we discuss is that $\delta(\mathbf{x})$ is a homogeneous and isotropic Gaussian random field. We briefly discuss 
non-Gaussian models in Section 5.1.

Statistical properties of a uniform and isotropic Gaussian field can be fully characterized by its power spectrum, $P(k)$, which depends only on the modulus $k$ of the wavevector, but not on its direction. A related quantity is the variance of the density contrast field smoothed on some scale $R: \delta_{R}(\mathbf{x}) \equiv \int \delta(\mathbf{x}-\mathbf{r}) W(\mathbf{r}, R) d^{3} r$, where

$$
\left\langle\delta_{R}^{2}\right\rangle \equiv \sigma^{2}(R)=\frac{1}{(2 \pi)^{3}} \int P(k)|\tilde{W}(\mathbf{k}, R)|^{2} d^{3} k
$$

where $\tilde{W}(\mathbf{k}, R)$ is the Fourier transform of the window (filter) function $W(\mathbf{r}, R)$, such that $\delta_{R}(\mathbf{k})=\delta(\mathbf{k}) \tilde{W}(\mathbf{k}, R)$ [see, e.g., Zentner (2007) or Mo, van den Bosch \& White (2010) for details on the definition of $P(k)$ and choices of window function]. For the cases, when one is interested in only a narrow range of $k$ the power spectrum can be approximated by the power-law form, $P(k) \propto k^{n}$, and the variance is $\sigma^{2}(R) \propto R^{-(n+3)}$.

At a sufficiently high redshift $\mathrm{z}$, for the spherical top-hat window function mass and radius are interchangeable according to the relation $M=4 \pi / 3 \rho_{\mathrm{m}}(\mathrm{z}) R^{3}$. We can think about the density field smoothed on the scale $R$ or the corresponding mass scale $M$. The characteristic amplitude of peaks in the $\delta_{R}$ (or $\delta_{M}$ ) field smoothed on scale $R$ (or mass scale $M$ ) is given by $\sigma(R) \equiv \sigma(M)$. The smoothed Gaussian density field is, of course, also Gaussian with the probability distribution function (PDF) given by

$$
p\left(\delta_{M}\right)=\frac{1}{\sqrt{2 \pi} \sigma(M)} \exp \left[-\frac{\delta_{M}^{2}}{2 \sigma^{2}(M)}\right] .
$$

During the earliest linear stages of evolution in the standard structure formation scenario the initial Gaussianity of the $\delta(\mathbf{x})$ field is preserved, as different Fourier modes $\delta(\mathbf{k})$ evolve independently and grow at the same rate, described by the linear growth factor, $D_{+}(a)$, as a function of expansion factor $a=(1+\mathrm{z})^{-1}$, which for a $\Lambda \mathrm{CDM}$ cosmology is given by Heath (1977):

$$
\delta(a) \propto D_{+}(a)=\frac{5 \Omega_{\mathrm{m}}}{2} E(a) \int_{0}^{a} \frac{d a^{\prime}}{\left[a^{\prime} E\left(a^{\prime}\right)\right]^{3}},
$$

where $E(a)$ is the normalized expansion rate, which is given by

$$
E(a) \equiv \frac{H(a)}{H_{0}}=\left[\Omega_{\mathrm{m}} a^{-3}+\left(1-\Omega_{\mathrm{m}}-\Omega_{\Lambda}\right) a^{-2}+\Omega_{\Lambda}\right]^{1 / 2},
$$

if the contribution from relativistic species, such as radiation or neutrinos, to the energydensity is neglected. Growth rate and the expression for $E(a)$ in more general, homogeneous dark energy (DE) cosmologies are described by Percival (2005). Note that in models in which DE is clustered (Alimi et al. 2010) or gravity deviates from General Relativity (GR, see Section 5.2), the growth factor can be scale dependent.

Correspondingly, the linear evolution of the root mean square (rms) amplitude of fluctuations is given by $\sigma(M, a)=\sigma\left(M, a_{i}\right) D_{+}(a) / D_{+}\left(a_{i}\right)$, which is often useful to recast in terms of linearly extrapolated rms amplitude $\sigma(M, a=1)$ at $a=1$ (i.e., z $=0$ ):

$$
\sigma(M, a)=\sigma(M, a=1) D_{+0}(a), \quad \text { where } D_{+0}(a) \equiv D_{+}(a) / D_{+}(a=1) .
$$


Once the amplitude of typical fluctuations approaches unity, $\sigma(M, a) \sim 1$, the linear approximation breaks down. Further evolution must be studied by means of nonlinear models or direct numerical simulations. We discuss results of numerical simulations extensively below. However, we consider first the simplified, but instructive, spherical collapse model and associated concepts and terminology. Such model can be used to gain physical insight into the key features of the evolution and is used as a basis for both definitions of collapsed objects (see Section 3.6) and quantitative models for halo abundance and clustering (Section 3.7 and 3.8).

\subsection{Non-linear evolution of spherical perturbations and non-linear mass scale}

The simplest model of non-linear collapse assumes that density peak can be characterized as constant overdensity spherical perturbation of radius $R$. Despite its simplicity and limitations discussed below, the model provides a useful insight into general features and timing of non-linear collapse. Its results are commonly used in analytic models for halo abundance and clustering and motivate mass definitions for collapsed objects. Below we briefly describe the model and non-linear mass scale that is based on its predictions.

\subsubsection{Spherical collapse model.}

The spherical collapse model considers a spherically-symmetric density fluctuation of initial radius $R_{\mathrm{i}}$, amplitude $\delta_{\mathrm{i}}>0$, and mass $M=(4 \pi / 3)\left(1+\delta_{\mathrm{i}}\right) \bar{\rho} R_{\mathrm{i}}^{3}$, where $R_{\mathrm{i}}$ is physical radius of the perturbation and $\bar{\rho}$ is the mean density of the Universe at the initial time. Given the symmetry, the collapse of such perturbation is a one-dimensional problem and

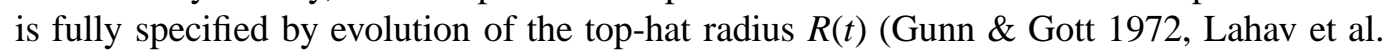
$1991)$. It consists of an initially decelerating increase of the perturbation radius, until it reaches the maximum value, $R_{\mathrm{ta}}$, at the turnaround epoch, $t_{\mathrm{ta}}$, and subsequent decrease of $R(t)$ at $t>t_{\mathrm{ta}}$ until the perturbation collapses, virializes, and settles at the final radius $R_{\mathrm{f}}$ at $t=t_{\text {coll }}$. Physically, $R_{\mathrm{f}}$ is set by the virial relation between potential and kinetic energy and is $R_{\mathrm{f}}=R_{\mathrm{ta}} / 2$ in cosmologies with $\Omega_{\Lambda}=0$. The turnaround epoch and the epoch of collapse and virialization are defined by initial conditions.

The final mean internal density of a collapsed object can be estimated by noting that in a $\Omega_{\Lambda}=0$ Universe the time interval $t_{\text {coll }}-t_{\mathrm{ta}}=t_{\mathrm{ta}}$ should be equal to the free-fall time of a uniform sphere $t_{\mathrm{ff}}=\sqrt{3 \pi /\left(32 G \rho_{\mathrm{ta}}\right)}$, which means that the mean density of perturbation at turnaround is $\rho_{\mathrm{ta}}=3 \pi /\left(32 G t_{\mathrm{ta}}^{2}\right)$ and $\rho_{\text {coll }}=8 \rho_{\mathrm{ta}}=3 \pi /\left(G t_{\text {coll }}^{2}\right)$. These densities can be compared with background mean matter densities at the corresponding times to get mean internal density contrasts: $\Delta=\rho / \bar{\rho}_{\mathrm{m}}$. In the Einstein-de Sitter model $\left(\Omega_{\mathrm{m}}=1, \Omega_{\Lambda}=0\right)$, background density evolves as $\bar{\rho}_{\mathrm{m}}=1 /\left(6 \pi G t^{2}\right)$, which means that density contrast after virialization is

$$
\Delta_{\mathrm{vir}} \equiv \frac{\rho_{\text {coll }}}{\bar{\rho}_{\mathrm{m}}}=18 \pi^{2}=177.653 .
$$

For general cosmologies, density contrast can be computed by estimating $\rho_{\text {coll }}$ and $\bar{\rho}_{m}\left(t_{\text {coll }}\right)$ in a similar fashion. For lower $\Omega_{\mathrm{m}}$ models, fluctuation of the same mass $M$ and $\delta$ has a larger initial radius and smaller physical density and, thus, takes longer to collapse. The density contrasts of collapsed objects therefore are larger in lower density models because the mean density of matter at the time of collapse is smaller. Accurate (to $\lesssim 1 \%$ for $\Omega_{\mathrm{m}}=0.1-1$ ) approximations for $\Delta_{\text {vir }}$ in open $\left(\Omega_{\Lambda}=0\right)$ and flat $\Lambda \mathrm{CDM}\left(1-\Omega_{\Lambda}-\Omega_{\mathrm{m}}=0\right)$ cosmologies 
are given by Bryan \& Norman (1998, their equation 6). For example, for the concordance $\Lambda \mathrm{CDM}$ cosmology with $\Omega_{\mathrm{m}}=0.27$ and $\Omega_{\Lambda}=0.73$ (Komatsu et al. 2011), density contrast at $\mathrm{z}=0$ is $\Delta_{\text {vir }} \approx 358$.

Note that if the initial density contrast $\delta_{\mathrm{i}}$ would grow only at the linear rate, $D_{+}(\mathrm{z})$, then the density contrast at the time of collapse would be more than a hundred times smaller. Its value can be derived starting from the density contrast linearly extrapolated to the turn around epoch, $\delta_{\mathrm{ta}}$. This epoch corresponds to the time at which perturbation enters in the non-linear regime and detaches from the Hubble expansion, so that $\delta_{\text {ta }} \sim 1$ is expected. In fact, the exact calculation in the case of $\Omega_{\mathrm{m}}(\mathrm{z})=1$ at the redshift of turn-around gives $\delta_{t a}=1.062$ (Gunn \& Gott 1972). Because $t_{\text {coll }}=2 t_{\text {ta }}$, further linear evolution for $\Omega_{\mathrm{m}}(\mathrm{z})=1$ until the collapse time gives $\delta_{c}=\delta_{\mathrm{ta}} D_{+}\left(t_{c}\right) / D_{+}\left(t_{\mathrm{ta}}\right) \approx 1.686$. In the case of $\Omega_{\mathrm{m}} \neq 1$ we expect that $\delta_{\mathrm{ta}}$ should have different values. For instance, for $\Omega_{\mathrm{m}}<1$ density contrast at turn-around should be higher to account for the higher rate of the Hubble expansion. However, linear growth from $t_{\mathrm{ta}}$ to $t_{\mathrm{coll}}$ is smaller due to the slower redshift dependence of $D_{+}(\mathrm{z})$. As a matter of fact, these two factors nearly cancel, so that $\delta_{c}$ has a weak dependence on $\Omega_{\mathrm{m}}$ and $\Omega_{\Lambda}$ (e.g., Percival 2005). For the concordance $\Lambda$ CDM cosmology at $\mathrm{z}=0$, for example, $\delta_{c} \approx 1.675$.

Additional interesting effects may arise in models with DE characterized by small or zero speed of sound, in which structure growth is affected not only because DE influences linear growth, but also because it participates non-trivially in the collapse of matter and may slow down or accelerate the formation of clusters of a given mass depending on DE equation of state (Abramo et al. 2007, Creminelli et al. 2010). DE in such models can also contribute non-trivially to the gravitating mass of clusters.

\subsubsection{The nonlinear mass scale $M_{\mathrm{NL}}$.}

The linear value of the collapse overdensity $\delta_{c}$ is useful in predicting whether a given initial perturbation $\delta_{i} \ll 1$ at initial $\mathrm{z}_{\mathrm{i}}$ collapses by some later redshift $\mathrm{z}$. The collapse condition is simply $\delta_{i} D_{+0}(\mathrm{z}) \geq \delta_{c}(\mathrm{z})$ and is used extensively to model the abundance and clustering of collapsed objects, as we discuss below in $\S 3.7$. The distribution of peak amplitudes in the initial Gaussian overdensity field smoothed over mass scale $M$ is given by a Gaussian PDF with a rms value of $\sigma(M)$ (Equation 2). The peaks in the initial Gaussian overdensity field smoothed at redshift $\mathrm{z}_{i}$ over mass scale $M$ can be characterized by the ratio $v=\delta_{i} / \sigma\left(M, \mathrm{z}_{i}\right)$ called the peak height. For a given mass scale $M$, the peaks collapsing at a given redshift $\mathrm{z}$ according to the spherical collapse model have the peak height given by:

$$
v \equiv \frac{\delta_{c}(\mathrm{z})}{\sigma(M, \mathrm{z})} .
$$

Given that $\delta_{c}(\mathrm{z})$ is a very weak function of $\mathrm{z}$ (changing by $\lesssim 1-2 \%$ typically), whereas $\sigma(M, \mathrm{z})=\sigma(M, \mathrm{z}=0) D_{+0}(\mathrm{z})$ decreases strongly with increasing $\mathrm{z}$, the peak height of collapsing objects of a given mass $M$ increases rapidly with increasing redshift.

Using Equation 7 we can define the characteristic mass scale for which a typical peak $(v=1)$ collapses at redshift $\mathrm{z}$ :

$$
\sigma\left(M_{\mathrm{NL}}, \mathrm{z}\right)=\sigma\left(M_{\mathrm{NL}}, \mathrm{z}=0\right) D_{+0}(\mathrm{z})=\delta_{c}(\mathrm{z})
$$

This nonlinear mass, $M_{\mathrm{NL}}(\mathrm{z})$, is a key quantity in the self-similar models of structure for- 
mation, which we consider in Section 3.9.

\subsection{Nonlinear collapse of real density peaks}

The spherical collapse model provides a useful approximate guideline for the time scale of halo collapse and has proven to be a very useful tool in developing approximate statistical models for the formation and evolution of halo populations. Such a simple model and its extensions (e.g., ellipsoidal collapse model) do, however, miss many important details and complexities of collapse of the real density peaks. Such complexities are usually explored using three-dimensional numerical cosmological simulations. Techniques and numerical details of such simulations are outside the scope of this review and we refer readers to recent reviews on this subject (Bertschinger 1998, Borgani \& Kravtsov 2011, Dolag et al. 2008, Norman 2010). Here, we simply discuss the main features of gravitational collapse learned from analyses of such simulations.

Figure 6 shows evolution of the DM density field in a cosmological simulation of a comoving region of $15 h^{-1} \mathrm{Mpc}$ on a side around cluster mass-scale density peak in the initial perturbation field from $\mathrm{z}=3$ to the present epoch. The overall picture is quite different from the top-hat collapse. First of all, real peaks in the primordial field do not have the constant density or sharp boundary of the top-hat, but have a certain radial profile and curvature (Bardeen et al. 1986, Dalal et al. 2008). As a result, different regions of a peak collapse at different times so that the overall collapse is extended in time and the peak does not have a single collapse epoch (e.g., Diemand, Kuhlen \& Madau 2007). Consequently, the distribution of matter around the collapsed peak can smoothly extend to several virial radii for late epochs and small masses (Cuesta et al. 2008, Prada et al. 2006). This creates ambiguity about the definition of halo mass and results in a variety of mass definitions adopted in practice, as we discuss in Section 3.6.

Second, the peaks in the smoothed density field, $\delta_{\mathrm{R}}(\mathbf{x})$, are not isolated but are surrounded by other peaks and density inhomogeneities. The tidal forces from the most massive and rarest peaks in the initial density field shepherd the surrounding matter into massive filamentary structures that connect them (Bond, Kofman \& Pogosyan 1996). Accretion of matter onto clusters at late epochs occurs preferentially along such filaments, as can be clearly seen in Figure 6.

Finally, the density distribution within the peaks in the actual density field is not smooth, as in the smoothed field $\delta_{\mathrm{R}}(\mathbf{x})$, but contains fluctuations on all scales. Collapse of density peaks on different scales can proceed almost simultaneously, especially during early stages of evolution in the CDM models when peaks undergoing collapse involve small scales, over which the power spectrum has an effective slope $n \approx-3$. Figure 6 shows that at high redshifts the proto-cluster region contains mostly small-mass collapsed objects, which merge to form a larger and larger virialized system near the center of the shown region at later epochs. Nonlinear interactions between smaller-scale peaks within a cluster-scale peak during mergers result in relaxation processes and energy exchange on different scales, and mass redistribution. Although the processes accompanying major mergers are not as violent as envisioned in the violent relaxation scenario (Valluri et al. 2007), such interactions lead to significant redistribution of mass (Kazantzidis, Zentner \& Kravtsov 2006) and angular momentum (Vitvitska et al. 2002, both within and outside of the virial radius. 

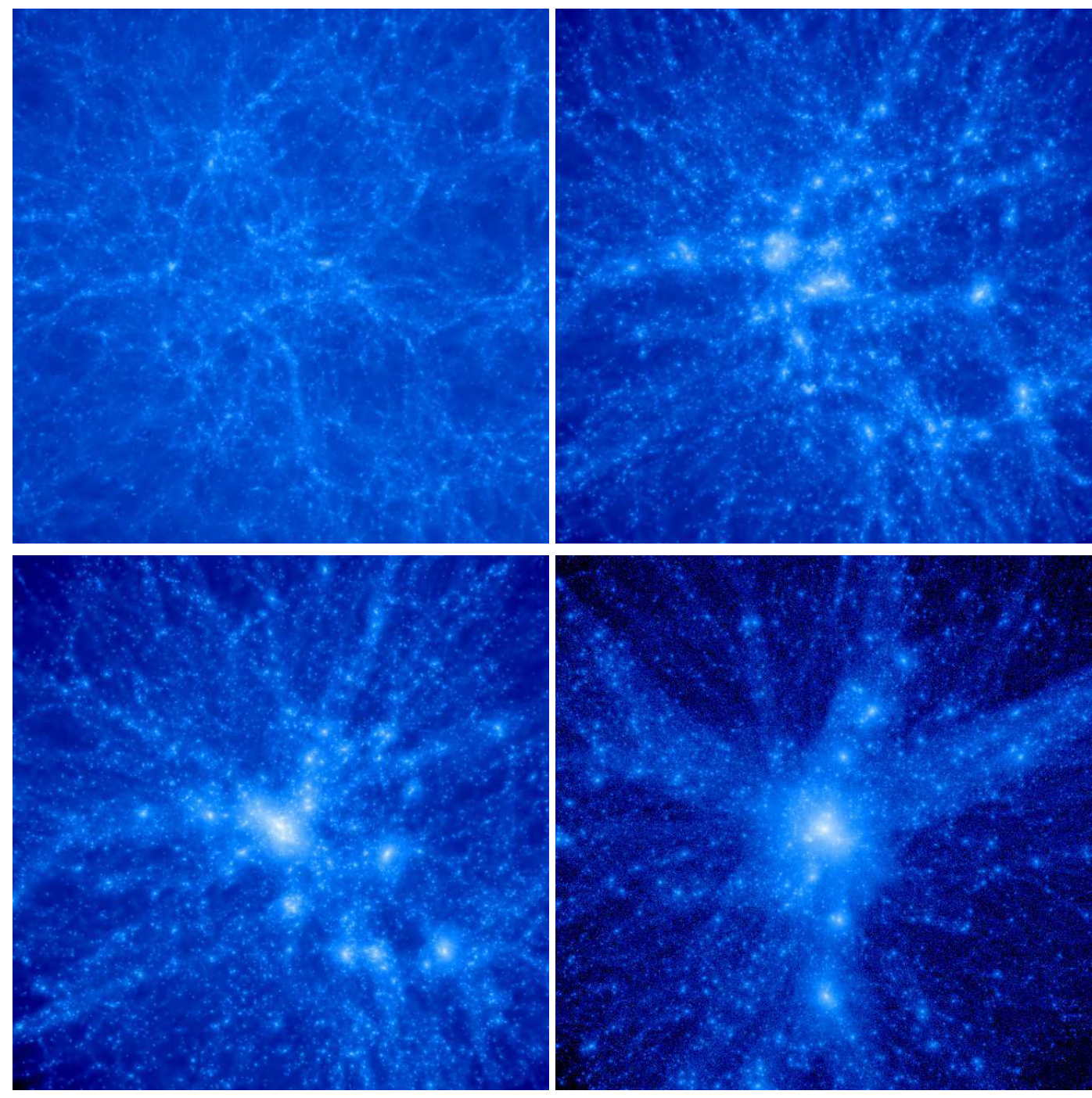

Fig. 6. Evolution of a dark matter density field in a comoving region of $15 h^{-1} \mathrm{Mpc}$ on a side around cluster mass density peak in the initial perturbation field. The four panels, from top left to bottom right, show redshifts $\mathrm{z}=3, \mathrm{z}=1, \mathrm{z}=0.5$ and $\mathrm{z}=0$. The forming cluster has a mass $M_{200} \simeq 1.2 \times 10^{15} h^{-1} M_{\odot}$ at $\mathrm{z}=0$. The figure illustrates the complexities of the actual collapse of real density peaks: strong deviations from spherical symmetry, accretion of matter along filaments, and the presence of smaller-scale structure within the collapsing cluster-scale mass peak.

\subsection{Equilibrium}

Following the collapse, matter settles into an equilibrium configuration. For collisional baryonic component this configuration is approximately described by the hydrostatic equilibrium (HE hereafter) equation, in which the pressure gradient $\nabla p(\mathbf{x})$ at point $\mathbf{x}$ is balanced by the gradient of local gravitational potential $\nabla \phi(\mathbf{x}): \nabla \phi(\mathbf{x})=-\nabla p(\mathbf{x}) / \rho_{\mathrm{g}}(\mathbf{x})$, where $\rho_{\mathrm{g}}(\mathbf{x})$ is the gas density. Under the further assumption of spherical symmetry, the HE equation 
can be written as $\rho_{\mathrm{g}}^{-1} d p / d r=-G M(<r) / r^{2}$, where $M(<r)$ is the mass contained within the radius $r$. Assuming the equation of state of ideal gas, $p=\rho_{\mathrm{g}} k_{B} T / \mu m_{p}$ where $\mu$ is the mean molecular weight and $m_{p}$ is the proton mass; cluster mass within $r$ can be expressed in terms of the density and temperature profiles, $\rho_{\mathrm{g}}(r)$ and $T(r)$, as

$$
M_{H E}(<r)=-\frac{r k_{B} T(r)}{G \mu m_{p}}\left[\frac{d \ln \rho_{\mathrm{g}}(r)}{d \ln r}+\frac{d \ln T(r)}{d \ln r}\right] .
$$

Interestingly, the slopes of the gas density and temperature profiles that enter the above equation exhibit correlation that appears to be a dynamical attractor during cluster formation (Juncher, Hansen \& Macciò 2012).

For a collisionless system of particles, such as CDM, the condition of equilibrium is given by the Jeans equation (e.g., Binney \& Tremaine 2008). For a non-rotating spherically symmetric system, this equation can be written as

$$
M_{J}(<r)=-\frac{r \sigma_{r}^{2}}{G}\left[\frac{d \ln v(r)}{d \ln r}+\frac{d \ln \sigma_{r}(r)^{2}}{d \ln r}+2 \beta(r)\right],
$$

where $\beta=1-\frac{\sigma_{t}^{2}}{2 \sigma_{r}^{2}}$ is the orbit anisotropy parameter defined in terms of the radial $\left(\sigma_{r}\right)$ and tangential $\left(\sigma_{t}\right)$ velocity dispersion components $(\beta=0$ for isotropic velocity field). We consider equilibrium density and velocity dispersion profiles, as well as anisotropy profile $\beta(r)$ in $\S 3.5 .2$. Equation 10 is also commonly used to describe the equilibrium of cluster galaxies. Although, in principle, galaxies in groups and clusters are not strictly collisionless, interactions between galaxies are relatively rare and the Jeans equation should be quite accurate.

Note that the difference between equilibrium configuration of collisional ICM and collisionless DM and galaxy systems is significant. In HE, the iso-density surfaces of the ICM should trace the iso-potential surfaces. The shape of the iso-potential surfaces in equilibrium is always more spherical than the shape of the underlying mass distribution that gives rise to the potential. Given that the potential is dominated by DM at most of the clustercentric radii, the ICM distribution (and consequently the X-ray isophotes and SZ maps) will be more spherical than the underlying DM distribution.

As we noted in the previous section, the gravitational collapse of a halo is a process extended in time. Consequently, a cluster may not reach complete equilibrium over the Hubble time due to ongoing accretion of matter and the occurrence of minor and major mergers. The ICM reaches equilibrium state following a major merger only after $\approx 3-4$ Gyr (e.g., Nelson et al. 2011). Deviations from equilibrium affect observable properties of clusters and cause systematic errors when equations 9 and 10 are used to estimate cluster masses (e.g., Ameglio et al. 2009; Lau, Kravtsov \& Nagai 2009; Nagai, Kravtsov \& Vikhlinin 2007; Piffaretti \& Valdarnini 2008; Rasia, Tormen \& Moscardini 2004).

\subsection{Internal structure of cluster halos}

Relaxations processes establish the equilibrium internal structure of clusters. Below we review our current undertstanding of the equilibrium radial density distribution, velocity dispersion, and triaxiality (shape) of the cluster DM halos. 


\subsubsection{Density Profile.}

Internal structure of collapsed halos may be expected to depend both on the properties of the initial density distribution around collapsing peaks (Hoffman \& Shaham 1985) and on the processes accompanying hierarchical collapse (e.g., Syer \& White 1998, Valluri et al. 2007). The fact that simulations have demonstrated that the characteristic form of the spherically averaged density profile arising in CDM models, characterized by the logarithmic slope steepening with increasing radius (Dubinski \& Carlberg 1991; Katz 1991; Navarro, Frenk \& White 1995, 1996), is virtually independent of the shape of power spectrum and background cosmology (Cole \& Lacey 1996; Huss, Jain \& Steinmetz 1999b; Katz 1991; Navarro, Frenk \& White 1997) is non trivial. Such a generic form of the profile also arises when small-scale structure is suppressed and the collapse is smooth, as is the case for halos forming at the cut-off scale of the power spectrum (Diemand, Moore \& Stadel 2005; Moore et al. 1999; Wang \& White 2009) or even from non-cosmological initial conditions (Huss, Jain \& Steinmetz 1999a).

The density profiles measured in dissipationless simulations are most commonly approximated by the "NFW" form proposed by Navarro, Frenk \& White (1995) based on their simulation of cluster formation:

$$
\rho_{\mathrm{NFW}}(r)=\frac{4 \rho_{s}}{x(1+x)^{2}}, \quad x \equiv r / r_{s}
$$

where $r_{s}$ is the scale radius, at which the logarithmic slope of the profile is equal to -2 and $\rho_{s}$ is the characteristic density at $r=r_{s}$. Overall, the slope of this profile varies with radius as $d \ln \rho / d \ln r=-[1+2 x /(1+x)]$, i.e., from the asymptotic slope of -1 at $x \ll 1$ to -3 at $x \gg 1$, where the enclosed mass diverges logarithmically: $M(<r)=M_{\Delta} f(x) / f\left(c_{\Delta}\right)$, where $M_{\Delta}$ is the mass enclosing a given overdensity $\Delta, f(x) \equiv \ln (1+x)-x /(1+x)$ and $c_{\Delta} \equiv R_{\Delta} / r_{s}$ is the concentration parameter. Accurate formulae for the conversion of mass of the NFW halos defined for different values of $\Delta$ are given in the appendix of Hu \& Kravtsov (2003).

Subsequent simulations (Graham et al. 2006, Merritt et al. 2006, Navarro et al. 2004) showed that the Einasto (1965) profile and other similar models designed to describe deprojection of the Sérsic profile (Merritt et al. 2006) provide a more accurate description of the DM density profiles arising during cosmological halo collapse, as well as profiles of bulges and elliptical galaxies (Cardone, Piedipalumbo \& Tortora 2005). The Einasto profile is characterized by the logarithmic slope that varies as a power law with radius:

$$
\rho_{\mathrm{E}}(r)=\rho_{s} \exp \left[\frac{2}{\alpha}\left(1-x^{\alpha}\right)\right], \quad x \equiv r / r_{s},
$$

where $r_{s}$ is again the scale radius at which the logarithmic slope is -2 , but now for the Einasto profile, $\rho_{s} \equiv \rho_{\mathrm{E}}\left(r_{s}\right)$, and $\alpha$ is an additional parameter that describes the power-law dependence of the logarithmic slope on radius: $d \ln \rho_{\mathrm{E}} / d \ln r=-2 x^{\alpha}$.

Note that unlike the NFW profile and several other profiles discussed in the literature, the Einasto profile does not have an asymptotic slope at small radii. The slope of the density profile becomes increasingly shallower at small radii at the rate controlled by $\alpha$. The parameter $\alpha$ varies with halo mass and redshift: at $\mathrm{z}=0$ galaxy-sized halos are described by $\alpha \approx 0.16$, whereas massive cluster halos are described by $\alpha \approx 0.2-0.3$; these values increase by $\sim 0.1$ by $\mathrm{z} \approx 3$ (Gao et al. 2008). Although $\alpha$ depends on mass and redshift (and thus also on the cosmology) in a non-trivial way, Gao et al. (2008, and see also Duffy et al. 
2008 showed that these dependencies can be captured as a universal dependence on the peak height $v=\delta_{c} / \sigma(M, \mathrm{z})$ (see Section 3.2.2 above): $\alpha=0.0095 v^{2}+0.155$. Finally, unlike the NFW profile, the total mass for the Einasto profile is finite due to the exponentially decreasing density at large radii. A number of useful expressions for the Einasto profile, such as mass within a radius, are provided by Cardone, Piedipalumbo \& Tortora (2005), Mamon \& Łokas (2005), and Graham et al. (2006).

The origin of the generic form of the density profile has recently been explored in detail by Lithwick \& Dalal (2011), who show that it arises due to two main factors: $(a)$ the density and triaxiality profile of the original peak and $(b)$ approximately adiabatic contraction of the previously collapsed matter due to deepening of the potential well during continuing collapse. Without adiabatic contraction the profile resulting from the collapse would reflect the shape of the initial profile of the peak. For example, if the initial profile of mean linear overdensity within radius $r$ around the peak can be described as $\bar{\delta}_{\mathrm{L}} \propto r_{\mathrm{L}}^{-\gamma}$, it can be shown that the resulting differential density profile after collapse without adiabatic contraction behaves as $\rho(r) \propto r^{-g}$, where $g=3 \gamma /(1+\gamma)$ (Fillmore \& Goldreich 1984). Typical profiles of initial density peaks are characterized by shallow slopes, $\gamma \sim 0-0.3$ at small radii, and very steep slopes at large radii (e.g., Dalal et al. 2008), which means that resulting profiles after collapse should have slopes varying from $g \approx 0-0.7$ at small radii to $g \approx 3$ at large radii.

However, Lithwick \& Dalal (2011) showed that contraction of particle orbits during subsequent accretion of mass interior to a given radius $r$ leads to a much more gradual change of logarithmic slope with radius, such that the regime within which $g \approx 0-0.7$ is shifted to very small radii $\left(r / r_{\text {vir }} \lesssim 10^{-5}\right)$, whereas at the radii typically resolved in cosmological simulations the logarithmic slope is in the range of $g \approx 1-3$, so that the radial dependence of the logarithmic slope $g(r)=d \ln \rho / d \ln r$ is in good qualitative agreement with simulation results. This contraction occurs because matter that is accreted by a halo at a given stage of its evolution can deposit matter over a wide range of radii, including small radii. The orbits of particles that accreted previously have to respond to the additional mass, and they do so by contracting. For example, for a purely spherical system in which mass is added slowly so that the adiabatic invariant is conserved, radii $r$ of spherical shells must decrease to compensate an increase of $M(<r)$. This model thus elegantly explains both the qualitative shape of density profiles observed in cosmological simulations and their universality. The latter can be expected because the contraction process crucial to shaping the form of the profile should operate under general collapse conditions, in which different shells of matter collapse at different times.

Although the model of Lithwick \& Dalal (2011) provides a solid physical picture of halo profile formation, it also neglects some of the processes that may affect details of the resulting density profile, most notably the effects of mergers. Indeed, major mergers lead to resonant dynamical heating of a certain fraction of collapsed matter due to the potential fluctuations and tidal forces that they induce. The amount of mass that is affected by such heating is significant (e.g., Valluri et al. 2007). In fact, up to 40\% of mass within the virial radii of merging halos may end up outside of the virial radius of the merger. This implies, for example, that virial mass is not additive in major mergers. Nevertheless, in practice the merger remnant retains the functional form of the density profiles of the merger progenitors (Kazantzidis, Zentner \& Kravtsov 2006), which means that major mergers do not lead to efficient violent relaxation.

Although the functional form of the density profile arising during halo collapse is generic 
for a wide variety of collapse conditions and models, initial conditions and cosmology do significantly affect the physical properties of halo profiles such as its characteristic density and scale radius (Navarro, Frenk \& White 1997). These dependencies are often discussed in terms of halo concentrations, $c_{\Delta} \equiv R_{\Delta} / r_{s}$. Simulations show that the scale radius is approximately constant during late stages of halo evolution (Bullock et al. 2001, Wechsler et al. 2002), but evolves as $r_{s}=c_{\min } R_{\Delta}$ during early stages, when a halo quickly increases its mass through accretion and mergers (Zhao et al. 2009, 2003). The minimum value of concentration is $c_{\min }=$ const $\approx 3-4$ for $\Delta=200$. For massive cluster halos, which are in the fast growth regime at any redshift, the concentrations are thus expected to stay approximately constant with redshift or may even increase after reaching a minimum (Klypin, Trujillo-Gomez \& Primack 2011; Prada et al. 2012).

The characteristic time separating the two regimes can be identified as the formation epoch of halos. This time approximately determines the value of the scale radius and the subsequent evolution of halo concentration. The initial conditions and cosmology determine the formation epoch and the typical mass accretion histories for halos of a given mass (Bullock et al. 2001; Navarro, Frenk \& White 1997; Zhao et al. 2009), and therefore determine the halo concentrations. Although these dependences are non-trivial functions of halo mass and redshift, they can also be encapsulated by a universal function of the peak height $v$ (Prada et al. 2012, Zhao et al. 2009).

Baryon dissipation and feedback are expected to affect the density profiles of halos appreciably, although predictions for these effects are far less certain than predictions of the DM distribution in the purely dissipationless regime. The main effect is contraction of DM in response to the increasing depth of the central potential during baryon cooling and condensation, which is often modelled under the assumption of slow contraction conserving adiabatic invariants of particle orbits (e.g., Barnes \& White 1984, Blumenthal et al. 1986, Ryden \& Gunn 1987, Zeldovich et al. 1980). The standard model of such adiabatic contraction assumes that DM particles are predominantly on circular orbits, and for each shell of DM at radius $r$ the product of the radius and the enclosed mass $r M(r)$ is conserved (Blumenthal et al. 1986). The model makes a number of simplifying assumptions and does not take into account effects of mergers. Nevertheless, it was shown to provide a reasonably accurate description of the results of cosmological simulations (Gnedin et al. 2004). Its accuracy can be further improved by relaxing the assumption of circular orbits and adopting an empirical ansätz, in which the conserved quantity is $r M(\bar{r})$, where $\bar{r}$ is the average radius along the particle orbit, instead of $r M(r)$ (Gnedin et al. 2004). At the same time, several recent studies showed that no single set of parameters of such simple models describes all objects that form in cosmological simulations equally well (Abadi et al. 2010; Gnedin et al. 2011; Gustafsson, Fairbairn \& Sommer-Larsen 2006; Tissera et al. 2010).

A more subtle but related effect is the increase of the overall concentration of DM within the virial radius of halos due to re-distribution of binding energy between DM and baryons during the process of cluster assembly (Rudd, Zentner \& Kravtsov 2008). The larger range of radii over which this effect operates makes it a potential worry for the precision constraints from the cosmic shear power spectrum (Jing et al. 2006; Rudd, Zentner \& Kravtsov 2008 ). This effect depends primarily on the fraction of baryons that condense into the central halo galaxies and may be mitigated by the blow-out of gas by efficient AGN or SN feedback (van Daalen et al. 2011). The effects of baryons on the overall concentration of mass distribution in clusters are thus uncertain, but can potentially increase halo concentration and thereby significantly enhance the cross section for strong lensing (Mead et al. 2010, Puchwein et al. 2005, Rozo et al. 2008) and affect statistics of strong lens distribution 
in groups and clusters (e.g., More et al. 2011).

A number of studies have derived observational constraints on density profiles of clusters and their concentrations (Buote et al. 2007; Ettori et al. 2010; Mandelbaum, Seljak \& Hirata 2008; Okabe et al. 2010; Pointecouteau, Arnaud \& Pratt 2005; Schmidt \& Allen 2007; Sereno \& Zitrin 2012; Umetsu et al. 2011a, b; Vikhlinin et al. 2006; Wojtak \& Łokas 2010). Although most of these studies find that the concentrations of galaxy clusters predicted by $\Lambda \mathrm{CDM}$ simulations are in the ballpark of values derived from observations, the agreement is not perfect and there is tension between model predictions and observations, which may be due to effects of baryon dissipation (e.g., Fedeli 2012; Rudd, Zentner \& Kravtsov 2008).

Some studies do find that the concordance cosmology predictions of the average cluster concentrations are somewhat lower than the average values derived from X-ray observations (Buote et al. 2007, Duffy et al. 2008, Schmidt \& Allen 2007). Moreover, lensing analyses indicate that the slope of the density profile in central regions of some clusters may be shallower than predicted (Newman et al. 2011, 2009; Sand et al. 2008, 2004; Tyson, Kochanski \& dell' Antonio 1998), whereas concentrations are considerably higher than both theoretical predictions and most other observational determinations from X-ray and WL analyses (Comerford \& Natarajan 2007, Oguri et al. 2012, 2009, Zitrin et al. 2011).

At this point, it is not clear whether these discrepancies imply serious challenges to the $\Lambda \mathrm{CDM}$ structure formation paradigm, unknown baryonic effects flattening the profiles in the centers, or unaccounted systematics in the observational analyses (e.g., Dalal \& Keeton 2003, Hennawi et al. 2007). When considering such comparisons, it is important to remember that density profiles in cosmological simulations are always defined with respect to the center defined as the global density peak or potential minimum, whereas in observations the corresponding location is not as unambiguous as in simulations and the choice of center may affect the derived slope.

It should be noted that improved theoretical predictions for cluster-sized systems generally predict larger concentrations for the most massive objects than do extrapolations of the concentration-mass relations from smaller mass objects (Bhattacharya, Habib \& Heitmann 2011; Prada et al. 2012; Zhao et al. 2009). In addition, as we noted above, the evolution predicted for the concentrations of these rarest objects is much weaker than $c \propto(1+\mathrm{z})$ found for smaller mass halos, so rescaling the concentrations of high-redshift clusters by $(1+\mathrm{z})$ factor, as is often done, could lead to an overestimate of their concentrations.

\subsubsection{Velocity dispersion profile and velocity anisotropy.}

Velocity dispersion profile is a halo property related to its density profile. Simulations show that this profile generally increases from the central value to a maximum at $r \approx r_{s}$ and slowly decreases outward (e.g., Cole \& Lacey 1996; Rasia, Tormen \& Moscardini 2004). One remarkable result illustrating the close connection between density and velocity dispersion is that for collapsed halos in dissipationless simulations the ratio of density to the cube of the rms velocity dispersion can be accurately described by a power law over at least three decades in radius (Taylor \& Navarro 2001): $Q(r) \equiv \rho / \sigma^{3} \propto r^{-\alpha}$ with $\alpha \approx 1.9$.

An important quantity underlying the measured velocity dispersion profile is the profile of the mean velocity, and the mean radial velocity, $\bar{v}_{\mathrm{r}}$, in particular. For a spherically symmetric matter distribution in HE, we expect $\bar{v}_{\mathrm{r}}=0$. Therefore, the profile of $\bar{v}_{\mathrm{r}}$ is a useful diagnostic of deviations from equilibrium at different radii. Simulations show that clusters at $\mathrm{z}=0$ 
generally have zero mean radial velocities within $r \approx R_{\text {vir }}$ and turn sharply negative between 1 and $\approx 3 R_{\mathrm{vir}}$, where density is dominated by matter infalling onto cluster (Cole \& Lacey 1996; Cuesta et al. 2008; Eke, Navarro \& Frenk 1998).

The distinguishing characteristic between gas and DM is the fact that gas has an isotropic velocity dispersion tensor on small scales, whereas DM in general does not. On large scales, however, both gas and DM may have velocity fields that are anisotropic. The degree of velocity anisotropy is commonly quantified by the anisotropy profile, $\beta(r)$ (see $\S 3.4$ ). DM anisotropy is mild: $\beta \approx 0-0.1$ near the center and increases to $\beta \approx 0.2-0.4$ near the virial radius (Cole \& Lacey 1996; Colín, Klypin \& Kravtsov 2000; Eke, Navarro \& Frenk 1998; Lemze et al. 2011; Rasia, Tormen \& Moscardini 2004). Interestingly, velocities exhibit substantial tangential anisotropy outside the virial radius in the infall region of clusters (Cuesta et al. 2008, Lemze et al. 2011). Another interesting finding is that the velocity anisotropy correlates with the slope of the density profile (Hansen \& Moore 2006), albeit with significant scatter (Lemze et al. 2011).

The gas component also has some residual motions driven by mergers and gas accretion along filaments. Gas velocities tend to have tangential anisotropy (Rasia, Tormen \& Moscardini 2004), because radial motions are inhibited by the entropy profile, which is convectively stable in general.

\subsubsection{Shape.}

Although the density structure of mass distribution in clusters is most often described by spherically averaged profiles, clusters are thought to collapse from generally triaxial density peaks (Bardeen et al. 1986, Doroshkevich 1970). The distribution of matter within halos resulting from hierarchical collapse is triaxial as well (Allgood et al. 2006, Cole \& Lacey 1996, Dubinski \& Carlberg 1991, Frenk et al. 1988, Jing \& Suto 2002, Kasun \& Evrard 2005, Warren et al. 1992, with triaxiality predicted by dissipationless simulations increasing with decreasing distance from halo center (Allgood et al. 2006). Triaxiality of halos decreases with decreasing mass and redshift (Allgood et al. 2006, Kasun \& Evrard 2005 in a way that again can be parameterized in a universal form as a function of peak height (Allgood et al. 2006). The major axis of the triaxial distribution of clusters is generally aligned with the filament connecting a cluster with its nearest neighbor of comparable mass (e.g., Lee et al. 2008, West \& Blakeslee 2000), which reflects the fact that a significant fraction of mass and mergers is occurring along such filaments (e.g., Lee \& Evrard 2007, Onuora \& Thomas 2000).

Jing \& Suto (2002) showed how the formalism of density distribution as a function of distance from cluster center can be extended to the density distribution in triaxial shells. Accounting for such triaxiality is particularly important in theoretical predictions and observational analyses of weak and strong lensing (Becker \& Kravtsov 2011; Clowe, De Lucia \& King 2004; Corless \& King 2007; Dalal \& Keeton 2003; Hennawi et al. 2007; Oguri et al. 2005). At the same time, it is important to keep in mind that, as with many other results derived mainly from dissipationless simulations, the physics of baryons may modify predictions substantially.

The shape of the DM distribution in particular is quite sensitive to the degree of central concentration of mass. As baryons condense towards the center to form a central galaxy within a halo, the DM distribution becomes more spherical (Dubinski 1994; Evrard, Summers \& Davis 1994; Kazantzidis et al. 2004; Tissera \& Dominguez-Tenreiro 1998). The 
effect increases with decreasing radius, but is substantial even at half of the virial radius (Kazantzidis et al. 2004). The main mechanism behind this effect lies in adiabatic changes of the shapes of particle orbits in response to more centrally concentrated mass distribution after baryon dissipation (Debattista et al. 2008, Dubinski 1994).

In considering effects of triaxiality, it is important to remember that triaxiality of the hot intracluster gas and DM distribution are different (gas is rounder, see, e.g., discussion in Lau et al. 2011, and references therein). This is one of the reasons why mass proxies defined within spherical aperture using observable properties of gas (see $\S 4$ below) exhibit small scatter and are much less sensitive to cluster orientation.

The observed triaxiality of the ICM can be used as a probe of the shape of the underlying potential (Lau et al. 2011) and as a powerful diagnostic of the amount of dissipation that is occurring in cluster cores (Fang, Humphrey \& Buote 2009) and of the mass of the central cluster galaxy (Lau et al. 2012).

\subsection{Mass definitions}

As we discussed above, the existence of a particular density contrast delineating a halo boundary is predicted only in the limited context of the spherical collapse of a density fluctuation with the top-hat profile (i.e., uniform density, sharp boundary). Collapse in such a case proceeds on the same time scale at all radii and the collapse time and "virial radius" are well defined. However, the peaks in the initial density field are not uniform in density, are not spherical, and do not have a sharp boundary. Existence of a density profile results in different times of collapse for different radial shells. Note also that even in the spherical collapse model the virial density contrast formally applies only at the time of collapse; after a given density peak collapses its internal density stays constant while the reference (i.e., either the mean or critical) density changes merely due to cosmological expansion. The actual overdensity of the collapsed top-hat initial fluctuations will therefore grow larger than the initial virial overdensity at $t>t_{\text {collapse }}$.

The triaxiality of the density peak makes the tidal effects of the surrounding mass distribution important. Absence of a sharp boundary, along with the effects of non-uniform density, triaxiality and nonlinear effects during the collapse of smaller scale fluctuations within each peak, result in a continuous, smooth outer density profile without a well-defined radial boundary. Although one can identify a radial range, outside of which a significant fraction of mass is still infalling, this range is fairly wide and does not correspond to a single welldefined radius (Cuesta et al. 2008; Eke, Navarro \& Frenk 1998). The boundary based on the virial density contrast is, thus, only loosely motivated by theoretical considerations.

The absence of a well-defined boundary of collapsed objects makes the definition of the halo boundary and the associated enclosed mass ambiguous. This explains, at least partly, the existence of various halo boundary and mass definitions in the literature. Below we describe the main two such definitions: the Friends-of-Friends (FoF) and spherical overdensity (SO, see also White 2001). The FoF mass definition is used almost predominantly in analyses of cosmological simulations of cluster formation, whereas the SO halo definition is used both in observational and simulation analyses, as well as in analytic models, such as the Halo Occupation Distribution (HOD) model. Although other definitions of the halo mass are discussed, theoretical mass determinations often have to conform to the observational definitions of mass. Thus, for example, although it is possible to define the entire 
mass that will ever collapse onto a halo in simulations Anderhalden \& Diemand 2011, Cuesta et al. 2008), it is impossible to measure this mass in observations, which makes it of interest only from the standpoint of the theoretical models of halo collapse.

\subsubsection{The Friends-of-Friends mass.}

Historically, the FoF algorithm was used to define groups and clusters of galaxies in observations (Einasto et al. 1984, Huchra \& Geller 1982, Press \& Davis 1982) and was adopted to define collapsed objects in simulations of structure formation (Davis et al. 1985), Einasto et al. 1984). The FoF algorithm considers two particles to be members of the same group (i.e., "friends"), if they are separated by a distance that is less than a given linking length. Friends of friends are considered to be members of a single group - the condition that gives the algorithm its name. The linking length, the only free parameter of the method, is usually defined in units of the mean interparticle separation: $b=l / \bar{l}$, where $l$ is the linking length in physical units and $\bar{l}=\bar{n}^{-1 / 3}$ is the mean interparticle separation of particles with mean number density of $\bar{n}$.

Attractive features of the FoF algorithm are its simplicity (it has only one free parameter), a lack of any assumptions about the halo center, and the fact that it does not assume any particular halo shape. Therefore, it can better match the generally triaxial, complex mass distribution of halos forming in the hierarchical structure formation models.

The main disadvantages of the FoF algorithm are the difficulty in theoretical interpretation of the FoF mass, and sensitivity of the FoF mass to numerical resolution and the presence of substructure. For the smooth halos resolved with many particles the FoF algorithm with $b=0.2$ defines the boundary corresponding to the fixed local density contrast of $\delta_{\mathrm{FoF}} \approx 81.62$ (More et al. 2011). Given that halos forming in hierarchical cosmologies have concentrations that depend on mass, redshift, and cosmology, the enclosed overdensity of the FoF halos also varies with mass, redshift and cosmology. Thus, for example, for the current concordance cosmology the FoF halos (defined with $b=0.2$ ) of mass $10^{11}-10^{15} M_{\odot}$ have enclosed overdensities of $\sim 450-350$ at $\mathrm{z}=0$ and converge to overdensity of $\sim 200$ at high redshifts where concentration reaches its minimum value of $c \approx 3-4$ (More et al. 2011). For small particle numbers the boundary of the FoF halos becomes "fuzzier" and depends on the resolution (and so does the FoF mass). Simulations most often have fixed particle mass and the number of particles therefore changes with halo mass, which means that properties of the boundary and mass identified by the FoF are mass dependent. The presence of substructure in well-resolved halos further complicates the resolution and mass dependence of the FoF-identified halos (More et al. 2011). Furthermore, it is well known that the FoF may spuriously join two neighboring distinct halos with overlapping volumes into a single group. The fraction of such neighbor halos that are "bridged" increases significantly with increasing redshift.

\subsubsection{The Spherical Overdensity mass.}

The spherical overdensity algorithm defines the boundary of a halo as a sphere of radius enclosing a given density contrast $\Delta$ with respect to the reference density $\rho$. Unlike the FoF algorithm the definition of an SO halo also requires a definition of the halo center. The common choices for the center in theoretical analyses are the peak of density, the minimum of the potential, the position of the most bound particle, or, more rarely, the center of mass. 
Given that the center and the boundary need to be found simultaneously, an iterative scheme is used to identify the SO boundary around a given peak. The radius of the halo boundary, $R_{\Delta_{c}}$, is defined by solving the implicit equation

$$
M(<r)=\frac{4 \pi}{3} \Delta \rho(\mathrm{z}) r^{3},
$$

where $M(<r)$ is the total mass profile and $\rho(\mathrm{z})$ is the reference physical density at redshift $\mathrm{z}$ and $r$ is in physical (not comoving) radius.

The choice of $\Delta$ and the reference $\rho$ may be motivated by theoretical considerations or by observational limitations. For example, one can choose to define the enclosed overdensity to be equal to the "virial" overdensity at collapse predicted by the spherical collapse model, $\Delta \rho=\Delta_{\text {vir,c }} \rho_{\text {crit }}$ (see Section 3.2). Note that in $\Omega_{\mathrm{m}} \neq 1$ cosmologies, there is a choice for reference density to be either the critical density $\rho_{\mathrm{cr}}(\mathrm{z})$ or the mean matter density $\rho_{\mathrm{m}}(\mathrm{z})$ and both are in common use. The overdensities defined with respect to these reference densities, which we denote here as $\Delta_{c}$ and $\Delta_{\mathrm{m}}$, are related as $\Delta_{\mathrm{m}}=\Delta_{c} / \Omega_{\mathrm{m}}(\mathrm{z})$. Note that $\Omega_{\mathrm{m}}(\mathrm{z})=$ $\Omega_{\mathrm{m} 0}(1+\mathrm{z})^{3} / E^{2}(\mathrm{z})$, where $E(\mathrm{z})$ is given by Equation 4 . For concordance cosmology, $1-$ $\Omega_{\mathrm{m}}(\mathrm{z})<0.1$ at $\mathrm{z} \geq 2$ and the difference between the two definitions decreases at these high redshifts. In observations, the choice may simply be determined by the extent of the measured mass profile. Thus, masses derived from X-ray data under the assumption of $\mathrm{HE}$ are limited by the extent of the measured gas density and temperature profiles and are therefore often defined for the high values of overdensity: $\Delta_{\mathrm{c}}=2,500$ or $\Delta_{\mathrm{c}}=500$.

The crucial difference from the FoF algorithm is the fact that the SO definition forces a spherical boundary on the generally non-spherical mass definition. In addition, spheres corresponding to different halos may overlap, which means that a certain fraction of mass may be double counted (although in practice this fraction is very small, see, e.g., discussion in $\S 2.2$ of Tinker et al. 2008).

The advantage of the SO algorithm is the fact that the SO-defined mass can be measured both in simulations and observational analyses of clusters. In the latter the SO mass can be estimated from the total mass profile derived from the hydrostatic and Jeans equilibrium analysis for the ICM gas and galaxies, respectively (see Section 3.4 above), or gravitational lensing analyses (e.g., Hoekstra 2007, Vikhlinin et al. 2006). Furthermore, suitable observables that correlate with the SO mass with scatter of $\lesssim 10 \%$ can be defined (see $\S 4$ below), thus making this mass definition preferable in the cosmological interpretation of observed cluster populations. The small scatter shows that the effects of triaxiality is quite small in practice. Note, however, that the definition of the halo center in simulations and observations may not necessarily be identical, because in observations the cluster center is usually defined at the position of the peak or the centroid of X-ray emission or SZ signal, or at the position of the BCG.

\subsection{Abundance of halos}

Contrasting predictions for the abundance and clustering of collapsed objects with the observed abundance and clustering of galaxies, groups, and clusters has been among the most powerful validation tests of structure formation models (e.g., Blumenthal et al. 1984, Kaiser 1984, 1986, Press \& Schechter 1974).

Although real clusters are usually characterized by some quantity derived from observa- 
tions (an observable), such as the X-ray luminosity, such quantities are generally harder to predict $a b$ initio in theoretical models because they are sensitive to uncertain physical processes affecting the properties of cluster galaxies and intracluster gas. Therefore, the predictions for the abundance of collapsed objects are usually quantified as a function of their mass, i.e., in terms of the mass function $\operatorname{dn}(M, \mathrm{z})$ defined as the comoving volume number density of halos in the mass interval $[M, M+d M]$ at a given redshift $\mathrm{z}$. The predicted mass function is then connected to the abundance of clusters as a function of an observable using a calibrated mass-observable relation (discussed in $\S 4$ below). Below we review theoretical models for halo abundance and underlying reasons for its approximate universality.

\subsubsection{The mass function and its universality.}

The first statistical model for the abundance of collapsed objects as a function of their mass was developed by Press \& Schechter (1974). The main powerful principle underlying this model is that the mass function of objects resulting from nonlinear collapse can be tied directly and uniquely to the statistical properties of the initial linear density contrast field $\delta(\mathbf{x})$.

Statistically, one can define the probability $F(M)$ that a given region within the initial overdensity field smoothed on a mass scale $M, \delta_{\mathrm{M}}(\mathbf{x})$, will collapse into a halo of mass $M$ or larger:

$$
F(M)=\int_{-1}^{\infty} p(\delta) C_{\text {coll }}(\delta) d \delta,
$$

where $p(\delta) d \delta$ is the PDF of $\delta_{\mathrm{M}}(\mathbf{x})$, which is given by Equation 2 for the Gaussian initial density field, and $C_{\text {coll }}$ is the probability that any given point $\mathbf{x}$ with local overdensity $\delta_{\mathrm{M}}(\mathbf{x})$ will actually collapse. The mass function can then be derived as a fraction of the total volume collapsing into halos of mass $(M, M+d M)$, i.e., $d F / d M$, divided by the comoving volume within the initial density field occupied by each such halo, i.e., $M / \bar{\rho}$ :

$$
\frac{d n(M)}{d M}=\frac{\bar{\rho}_{\mathrm{m}}}{M}\left|\frac{d F}{d M}\right|
$$

In their pioneering model, Press \& Schechter (1974) have adopted the ansätz motivated by the spherical collapse model (see $\S 3.1$ ) that any point in space with $\delta_{\mathrm{M}}(\mathbf{x}) D_{+0}(\mathrm{z}) \geq \delta_{c}$ will collapse into a halo of mass $\geq M$ by redshift z: i.e., $C_{\text {coll }}(\delta)=\Theta\left(\delta-\delta_{c}\right)$, where $\Theta$ is the Heaviside step function. Note that $\delta_{\mathrm{M}}(\mathbf{x})$ used above is not the actual initial overdensity, but the initial overdensity evolved to $\mathrm{z}=0$ with the linear growth rate. One can easily check that for a Gaussian initial density field this assumption gives $F(M)=\frac{1}{2} \operatorname{erfc}\left[\delta_{c} /(\sqrt{2} \sigma(M, \mathrm{z}))\right]=$ $F(v)$. This line of arguments and assumptions thus leads to an important conclusion that the abundance of halos of mass $M$ at redshift $\mathrm{z}$ is a universal function of only their peak height $v(M, \mathrm{z}) \equiv \delta_{c} / \sigma(M, \mathrm{z})$. In particular, the fraction of mass in halos per logarithmic interval of mass in such a model is:

$$
\frac{d n(M)}{d \ln M}=\frac{\bar{\rho}_{\mathrm{m}}}{M}\left|\frac{d F}{d \ln M}\right|=\frac{\bar{\rho}_{\mathrm{m}}}{M}\left|\frac{d \ln v}{d \ln M} \frac{\partial F}{\partial \ln v}\right| \equiv \frac{\bar{\rho}_{\mathrm{m}}}{M}\left|\frac{d \ln v}{d \ln M}\right| g(v) \equiv \frac{\bar{\rho}_{\mathrm{m}}}{M} \psi(v) .
$$

Clearly, the shape $\psi(v)$ in such models is set by the assumptions of the collapse model. Numerical studies based on cosmological simulations have eventually revealed that the 


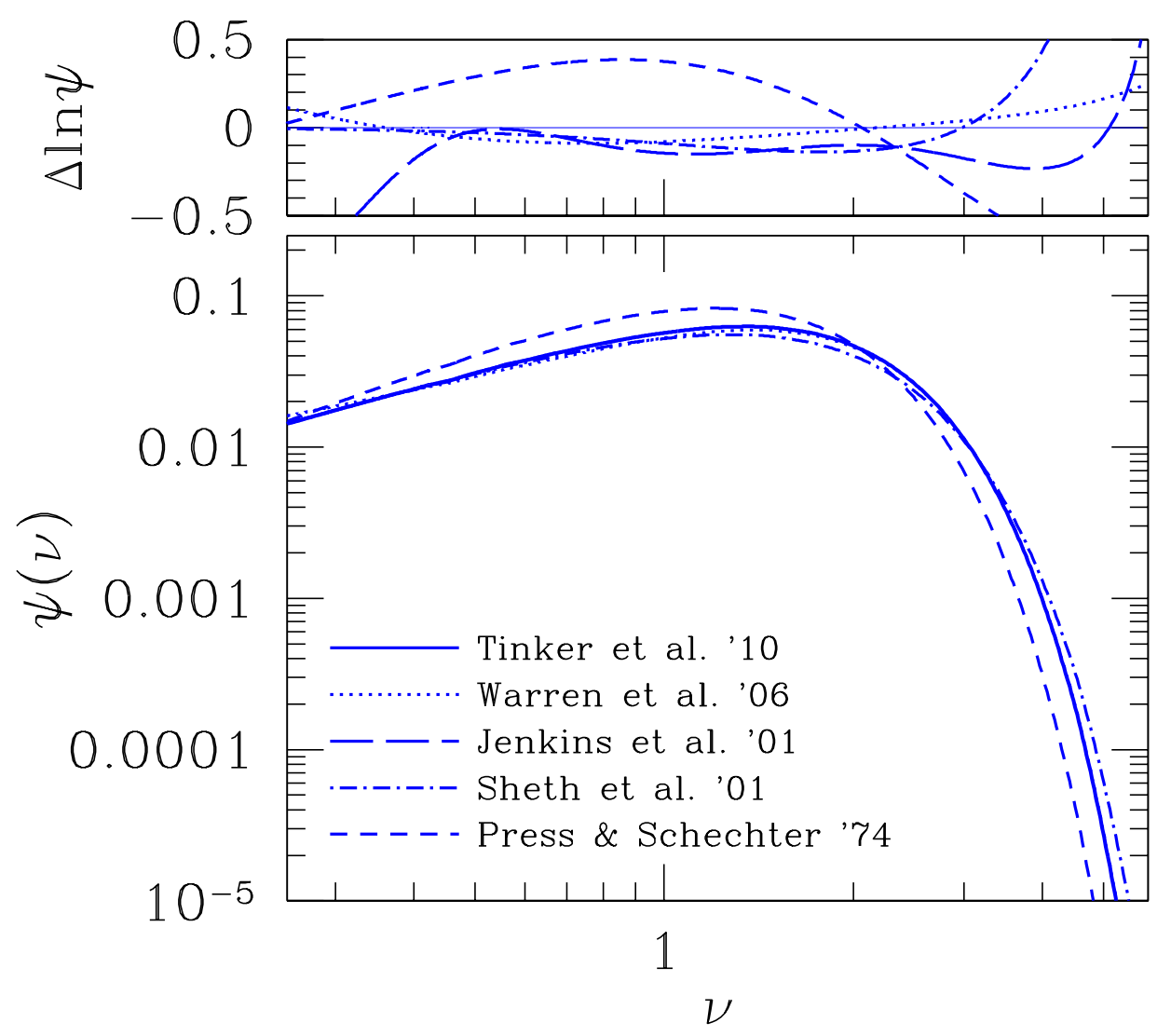

Fig. 7. The function $\psi(v)$ defining the comoving abundance of collapsed halos via $d n / d \ln M=\left(\bar{\rho}_{\mathrm{m}} / M\right) \psi(v)$ as a function of $v$ from different models and simulation-based calibrations. The upper panel shows deviations of specific models and calibrations for $\mathrm{z}=0$ from Tinker et al. (2010) based on a suite of $\Lambda$ CDM cosmological simulations.

shape $\psi_{\mathrm{PS}}(v)$ predicted by the Press \& Schechter (1974) model deviates by $\gtrsim 50 \%$ from the actual shape measured in cosmological simulations (e.g., Gross et al. 1998, Jenkins et al. 2001, Klypin et al. 1995, Lee \& Shandarin 1999, Sheth \& Tormen 1999, Tormen 1998).

A number of modifications to the original ansätz have been proposed, which result in $\psi(v)$ that more accurately describes simulation results. Such modifications are based on the collapse conditions that take into account asphericity of the peaks in the initial density field (Audit, Teyssier \& Alimi 1997; Desjacques 2008; Lee \& Shandarin 1998; Monaco 1995; Sheth \& Tormen 2002 and stochasticity due to the dependence of the collapse condition on peak properties other than $v$ or shape (e.g., Corasaniti \& Achitouv 2011; de Simone, Maggiore \& Riotto 2011; Desjacques 2008; Ma et al. 2011; Maggiore \& Riotto 2010). The more sophisticated excursion set models match the simulations more closely, albeit at the expense of more assumptions and parameters. There may be also inherent limitations in the accuracy of such models given that they rely on the strong assumption that one can parameterize all the factors influencing collapse of any given point in the initial overdensity field in a relatively compact form. In the face of complications to a simple picture of peak collapse, as discussed in Section 3.3, one can indeed expect that the excursion set ansätze 


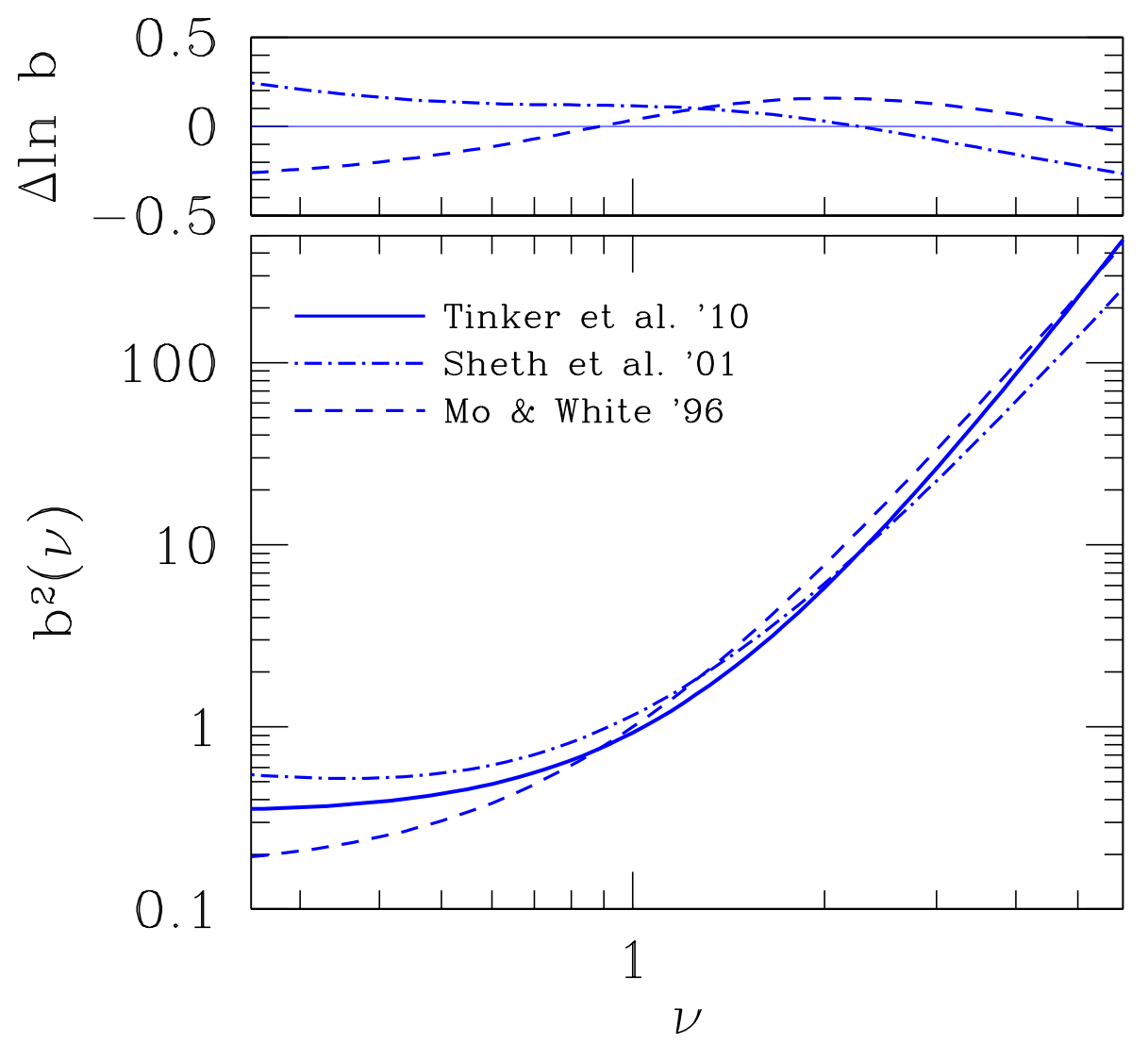

Fig. 8. The square of the bias, $b^{2}(v)$ as a function of peak height $v$ corresponding to halos of mass $M_{200 \mathrm{~m}}$ in the bias model based excursion set and spherical collapse barrier (dashed line Mo \& White 1996), in the excursion set model based on model of ellipsoidal collapse (dot-dashed line Sheth, Mo \& Tormen 2001), and the bias function calibrated using $\Lambda$ CDM cosmological simulations (solid line Tinker et al. 2010) for SO halos defined using overdensity of $\Delta=200$ with respect to the mean density. The upper panel shows deviations of excursion set models from the calibration of Tinker et al. (2010).

are limited in how accurately they can ultimately describe the halo mass function.

\subsubsection{Calibrations of halo mass function in cosmological simulations.}

An alternative route to derive predictions for halo abundance accurately is to calibrate it using large cosmological simulations of structure formation. Simulations have generally confirmed the remarkable fact that the abundance of halos can be parameterized via a universal function of peak height $v$ (Bhattacharya et al. 2011, Courtin et al. 2011, Crocce et al. 2010, Evrard et al. 2002, Jenkins et al. 2001, Lukić et al. 2007, Reed et al. 2007, Sheth $\&$ Tormen 1999, Tinker et al. 2008, Warren et al. 2006, White 2002). Note that in many studies the linear overdensity for collapse is assumed to be constant across redshifts and cosmologies and the mass function is therefore quantified as a function of $\sigma^{-1}$ - the quantity proportional to $v$. However, as pointed out by Courtin et al. (2011) it is necessary to 
include the redshift and cosmology dependence of $\delta_{c}(\mathrm{z})$ for an accurate description of the mass function across cosmologies. Even though $\delta_{c}$ varies only by $\sim 1-2 \%$, it enters into halo abundance via an exponent and such small variations can result in variations in the mass function of several per cent or more.

The main efforts with simulations have thus been aimed at improving the accuracy of the $\psi(v)$ functional form, assessing systematic uncertainties related to the mass definition, and quantifying deviations from the universality of $\psi(v)$ for different redshifts and cosmologies. The mass function, and especially its exponential tail, is sensitive to the specifics of halo mass definition, a point emphasized strongly in a number of studies (Cohn \& White 2008; Jenkins et al. 2001; Klypin, Trujillo-Gomez \& Primack 2011; Tinker et al. 2008; White 2002). Thus, in precision cosmological analyses using an observed cluster abundance, care must be taken to ensure that the cluster mass definition matches that used in the calibration of the halo mass function.

Predictions for the halo abundance as a function of the SO mass for a variety of overdensities used to define the SO boundaries, accurate to better than $\approx 5-10 \%$ over the redshift interval $\mathrm{z}=[0,2]$, were presented by Tinker et al. (2008). In Figure 7 we compare the form of the function $\psi(v)$ calibrated through simulations by different researchers and compared to $\psi(v)$ predicted by the Press-Schechter model, and to the calibration of the functional form based on the ellipsoidal collapse ansätz by Sheth, Mo \& Tormen (2001).

These calibrations of the mass function through $\mathrm{N}$-body simulations provide the basis for the use of galaxy clusters as tools to constrain cosmological models through the growth rate of perturbations (see the recent reviews by Allen, Evrard \& Mantz 2011 and Weinberg et al. 2012). As we discuss in Section 5 below, similar calibrations can be extended to models with non-Gaussian initial density field and models of modified gravity.

Future cluster surveys promise to provide tight constraints on cosmological parameters, thanks to the large statistics of clusters with accurately inferred masses. The potential of such surveys clearly requires a precise calibration of the mass function, which currently represents a challenge. Deviations from universality at the level of up to $\sim 10 \%$ have been reported (Cohn \& White 2008, Courtin et al. 2011, Crocce et al. 2010, Lukić et al. 2007, Reed et al. 2007, Tinker et al. 2008). In principle, a precise calibration of the mass function is a challenging but tractable technical problem, as long as it only requires a large suite of dissipationless simulations for a given set of cosmological parameters, and an optimal interpolation procedure (e.g., Lawrence et al. 2010).

A more serious challenge is the modelling of uncertain effects of baryon physics: baryon collapse, dissipation, and dynamical evolution, as well as feedback effects related to energy release by the SNe and AGN, may lead to subtle redistribution of mass in halos. Such redistribution can affect halo mass and thereby halo mass function at the level of a few per cent (Cui et al. 2011; Rudd, Zentner \& Kravtsov 2008; Stanek, Rudd \& Evrard 2009), although the exact magnitude of the effect is not yet certain due to uncertainties in our understanding of the physics of galaxy formation in general, and the process of condensation and dynamical evolution in clusters in particular.

\subsection{Clustering of halos}

Galaxy clusters are clustered much more strongly than galaxies themselves. It is this strong clustering discovered in the early 1980s (Bahcall \& Soneira 1983, Klypin \& Kopylov 1983) 
that led to the development of the concept of bias in the context of Gaussian initial density perturbation field (Kaiser 1984). Linear bias of halos is the coefficient between the overdensity of halos within a given sufficiently large region and the overdensity of matter in that region: $\delta_{\mathrm{h}}=b \delta$, with $b$ defined as the bias parameter. For the Gaussian perturbation fields, local linear bias is independent of scale (Scherrer \& Weinberg 1998), such that the largescale power spectrum and correlation function on large scales can be expressed in terms of the corresponding quantities for the underlying matter distribution as $P_{\mathrm{hh}}(k)=b^{2} P_{\mathrm{mm}}(k)$ and $\xi_{\mathrm{hh}}(r)=b^{2} \xi_{\mathrm{mm}}(r)$, respectively. As we discuss in Section 5, this is not true for nonGaussian initial perturbation fields (Dalal et al. 2008) or for models with scale-dependent linear growth rate (Parfrey, Hui \& Sheth 2011), in which cases the linear bias is generally scale-dependent.

In the context of the hierarchical structure formation, halo bias is closely related to the overall abundance of halos discussed above, as illustrated by the "peak-background split" framework (Cole \& Kaiser 1989, Kaiser 1984, Mo \& White 1996, Sheth \& Tormen 1999), in which the linear halo bias is obtained by considering a Lagrangian patch of volume $V_{0}$, mass $M_{0}$, and overdensity $\delta_{0}$ at some early redshift $z_{0}$. The bias is calculated by requiring that the abundance of collapsed density peaks within such a patch is described by the same function $\psi\left(v_{p}\right)$ as the mean abundance of halos in the Universe, but with peak height $v_{p}$ appropriately rescaled with respect to the overdensity of the patch and relative to the rms fluctuations on the scale of the patch. Thus, the functional form of the bias dependence on halo mass, $b_{h}(M)$, depends on the functional form of the mass function explicitly in this framework. Simulations show that the peak-background split model provides a fairly accurate (to $\sim 20 \%$ ) prediction for the linear halo bias (Tinker et al. 2010).

Another line of argument illustrating the close connection between the halo abundance and bias is the fact that if one assumes that all of the mass is in the collapsed halos, as is done for example in the halo models (Cooray \& Sheth 2002), the requirement that matter in the Universe is not biased against itself implies that $\int b(v) g(v) d \ln v=1$ (e.g., Seljak 2000), where $g(v) \equiv|d \ln v / d \ln M|^{-1} \psi(v)$ (see eq. 16). This integral constraint requires that the form of the bias function $b(v)$ is changed whenever $\psi(v)$ changes. Incidentally, the close connection between $b(v)$ and $\psi(v)$ implies that if $\psi(v)$ is a universal function, then the bias $b(v)$ should be a universal function as well.

The function $b(v)$ recently calibrated for the SO-defined halos of different overdensities using a suite of large cosmological simulations with accuracy $\lesssim 5 \%$ and satisfying the integral constraint (Tinker et al. 2010) is shown in Figure 8 for halos defined using an overdensity of $\Delta=200$ with respect to the mean. This calibration of the bias is compared to the corresponding prediction of the Press \& Schechter (1974) and the Sheth, Mo \& Tormen (2001) ansätze. The figure shows that $b(v)$ is a rather weak function of $v$ at $v<1$, but steepens substantially for rare peaks of $v>1$. It also shows that the rarest clusters $(v \sim 5)$ in the Universe can have the amplitude of the correlation function or power spectrum that is two orders of magnitude larger than the clustering amplitude of the galaxy-sized halos $(v \lesssim 1)$.

\subsection{Self-similar evolution of galaxy clusters}

In the previous sections we have considered processes that govern the collapse of matter during cluster formation, the transition to equilibrium and the equilibrium structure of matter distribution in collapsed halos. In the following sections, we consider baryonic pro- 
cesses that shape the observable properties of clusters, such as their X-ray luminosity or the temperature of the ICM. However, before we delve into the complexities of such physical processes, it is instructive to introduce the simplest models based on assumptions of selfsimilarity, in which the number of control parameters is minimal. We discuss the assumptions and predictions of the self-similar model in some detail because parametric scalings that it predicts are in wide use to interpret results from both cosmological simulations of cluster formation and observations.

\subsubsection{Self-similar model: assumptions and basic expectations.}

The self-similar model developed by Kaiser (1986) makes three key assumptions. The first assumption is that clusters form via gravitational collapse from peaks in the initial density field in an Einstein-de-Sitter Universe, $\Omega_{m}=1$. Gravitational collapse in such a Universe is scale-free, or self-similar. The second assumption is that the amplitude of density fluctuations is a power-law function of their size, $\Delta(k) \propto k^{3+n}$. This means that initial perturbations also do not have a preferred scale (i.e., they are scale-free or self-similar). The third assumption is that the physical processes that shape the properties of forming clusters do not introduce new scales in the problem. With these assumptions the problem has only two control parameters: the normalization of the power spectrum of the initial density perturbations at an initial time, $t_{\mathrm{i}}$, and its slope, $n$. Properties of the density field and halo population at $t>t_{\mathrm{i}}$ (or corresponding redshift $\mathrm{z}<\mathrm{z}_{\mathrm{i}}$ ), such as typical halo masses that collapse or halo abundance as a function of mass, depend only on these two parameters. One can choose any suitable variable that depends on these two parameters as a characteristic variable for a given problem. For evolution of halos and their abundance, the commonly used choice is to define the characteristic nonlinear mass, $M_{\mathrm{NL}}$ (see Section 3.1), which encapsulates such dependence. The halo properties and halo abundance then become universal functions of $\mu \equiv M / M_{\mathrm{NL}}$ in such model. Thus, for example, clusters with masses $M_{1}\left(\mathrm{z}_{1}\right)$ and $M_{2}\left(\mathrm{z}_{2}\right)$ that correspond to the same ratio $M_{1}\left(\mathrm{z}_{1}\right) / M_{\mathrm{NL}}\left(\mathrm{z}_{1}\right)=M_{2}\left(\mathrm{z}_{2}\right) / M_{\mathrm{NL}}\left(\mathrm{z}_{2}\right)$ have the same dimensionless properties, such as gas fraction or concentration of their mass distribution.

As we have discussed above, in more general cosmologies the halo properties and mass function should be universal functions of the peak height $v$, which encapsulates the dependence on the shape and normalization of the power spectra for general, non power-law shapes of the fluctuation spectrum.

\subsubsection{The Kaiser model for cluster scaling relations.}

In the this Section, we define cluster mass to be the mass within the sphere of radius $R$, encompassing characteristic density contrast, $\Delta$, with respect to some reference density $\rho_{r}$ (usually either $\rho_{\mathrm{cr}}$ or $\left.\rho_{\mathrm{m}}\right): M=(4 \pi / 3) \Delta \rho_{r} R^{3}$. In this definition, radius and mass are directly related and interchangeable. The model assumes spherical symmetry and that the ICM is in equilibrium within the cluster gravitational potential, so that the HE equation (eq. 9) holds. The mass $M(<R)$ derived from the HE equation is proportional to $T(R) R$ and the sum of the logarithmic slopes of the gas density and temperature profiles at $R$. In addition to the assumptions about self-similarity discussed above, a key assumption made in the model by 
Kaiser (1986) is that these slopes are independent of $M$, so that

$$
T \propto \frac{M}{R} \propto\left(\Delta \rho_{r}\right)^{\frac{1}{3}} M^{\frac{2}{3}}
$$

Note that formally the quantity $T$ appearing in the above equation is the temperature measured at $R$, whereas some average temperature at smaller radii is usually measured in observations. However, if we parameterize the temperature profile as $T(r)=T_{*} \tilde{T}(x)$, where $T_{*}$ is the characteristic temperature and $\tilde{T}$ is the dimensionless profile as a function of dimensionless radius $x \equiv r / R$, and we assume that $\tilde{T}(x)$ is independent of $M$, any temperature averaged over the same fraction of radial range $\left[x_{1}, x_{2}\right]$ will scale as $\propto T_{*} \propto T(R)$. The latter is not strictly true for the "spectroscopic" temperature, $T_{\mathrm{X}}$, derived by fitting an observed X-ray spectrum to a single-temperature bremsstrahlung model (Mazzotta et al. 2004, Vikhlinin 2006), although in practice deviations of $T_{\mathrm{X}}$ from the expected behavior for $T_{*}$ are small.

The gas mass within $R$ can be computed by integrating over the gas density profile, which, by analogy with temperature, we parameterize as $\rho_{\mathrm{g}}(r)=\rho_{\mathrm{g} *} \tilde{\mathrm{g}}_{\mathrm{g}}(x)$, where $\rho_{\mathrm{g} *}$ is the characteristic density and $\tilde{\rho}_{\mathrm{g}}$ is the dimensionless profile. The gas mass within $R$ can then be expressed as

$$
M_{\mathrm{g}}(<R)=4 \pi \rho_{\mathrm{g} *} R^{3} \int_{0}^{1} x^{2} \tilde{\rho}_{\mathrm{g}}(x) d x=3 M \frac{\rho_{\mathrm{g} *}}{\Delta \rho_{r}} I_{\rho_{\mathrm{g}}} \propto M(<R) .
$$

The latter proportionality is assumed in the Kaiser (1986) model, which means that $\rho_{\mathrm{g} *}$ and $I_{\rho_{\mathrm{g}}}$ are assumed to be independent of $M$. Note that $\rho_{\mathrm{g} *} \propto \Delta \rho_{r}$, so $\Delta \rho_{r}$ does not enter the $M_{\mathrm{g}}-M$ relation.

Using the scalings of $M_{\mathrm{g}}$ and $T$ with mass, we can construct other cluster properties of interest, such as the luminosity of ICM emitted due to its radiative cooling. Assuming that the ICM emission is due to the free-free radiation and neglecting the weak logarithmic dependence of the Gaunt factor on temperature, the bolometric luminosity can be written as (e.g., Sarazin 1986):

$$
L_{\mathrm{bol}} \propto \rho_{\mathrm{g}}^{2} T^{\frac{1}{2}} V \propto \frac{M_{\mathrm{g}}^{2}}{V} T^{\frac{1}{2}} \propto \Delta^{\frac{7}{6}} M^{\frac{4}{3}} .
$$

We omit $\rho_{r}$ in these equations for clarity; it suffices to remember that $\rho_{r}$ enters into the scaling relations exactly as $\Delta$. Note that the bolometric luminosity of a cluster is not observable directly, and the X-ray luminosity in soft band (e.g., $0.5-2 \mathrm{keV}), L_{\mathrm{Xs}}$, is frequently used. Such soft band X-ray luminosity is almost insensitive to temperature at $T>2 \mathrm{keV}$ (Fabricant \& Gorenstein 1983, as can be easily verified with a plasma emission code), so that its temperature dependence can be neglected. $L_{\mathrm{Xs}}$ then scales as:

$$
L_{\mathrm{Xs}} \propto \rho_{\mathrm{g}}^{2} V \propto \frac{M_{\mathrm{g}}^{2}}{V} \propto \Delta M .
$$

At temperatures $T<2 \mathrm{keV}$ temperature dependence is more complicated both for the bolometric and soft X-ray emissivity due to significant flux in emission lines. Therefore, strictly speaking, for lower mass systems the above $L-M$ scaling relations are not applicable, and scaling of the emissivity with temperature needs to be calibrated separately taking also into account the ICM metallicity. The same is true for luminosity defined in some other energy band or for the bolometric luminosity. 
Another quantity of interest is the ICM "entropy" defined in X-ray analyses as

$$
K_{\mathrm{X}} \equiv \frac{k_{\mathrm{B}} T_{\mathrm{X}}}{n_{e}^{2 / 3}} \propto \rho_{\mathrm{g}}^{-2 / 3} T \propto \Delta^{-1 / 3} M^{2 / 3} .
$$

where $n_{e}$ is the electron number density. Finally, the quantity, $Y=M_{\mathrm{g}} T$ where gas mass and temperature are measured within a certain range of radii scaled to $R_{\Delta}$, is used to characterize the ICM in the analyses of SZ and X-ray observations. This quantity is expected to be a particularly robust proxy of the cluster mass (e.g., da Silva et al. 2004; Fabjan et al. 2011; Kravtsov, Vikhlinin \& Nagai 2006; Motl et al. 2005; Nagai 2006, see also discussion in Section 4) because it is proportional to the global thermal energy of ICM. Using Equations 18 and 17 the scaling of $Y$ with mass in the self-similar model is

$$
Y \equiv M_{\mathrm{g}} T \propto \Delta^{1 / 3} M^{5 / 3} .
$$

Note that the redshift dependence in the normalization of the scaling relations introduced above is due solely to the particular SO definition of mass and associated redshift dependence of $\Delta \rho_{r}$. In $\Omega_{\mathrm{m}} \neq 1$ cosmologies, there is a choice of either defining the mass relative to the mean matter density or critical density (Section 3.6.2). This specific, arbitrary choice determines the specific redshift dependence of the observable-mass relations. It is clear that this evolution due to $\Delta(\mathrm{z})$ factors has no deep physical meaning. However, the absence of any additional redshift dependence in the normalization of the scaling relations is just the consequence of the assumptions of the Kaiser (1986) model and is a physical reflection of these assumptions.

Extra evolution can, therefore, be expected if one or more of the assumptions of the selfsimilar model is violated. This can be due to either actual physical processes that break self-similarity or the fact that some of the model assumptions are not accurate. We discuss physical processes that lead to the breaking of self-similarity in subsequent sections. Here below we first consider possible deviations that may arise because assumptions of the Kaiser model do not hold exactly, i.e. deviations not ascribed to physical processes that explicitly violate self-similarity.

\subsubsection{Extensions of the Kaiser model.}

Going back to equations 17 and 18, we note that the specific scaling of $T \propto M^{2 / 3}$ and $M_{\mathrm{g}} \propto M$ will only hold, if the assumption that the dimensionless temperature and gas density profiles, $\tilde{T}(x)$ and $\tilde{\rho}_{\mathrm{g}}(x)$, are independent of $M$ holds. In practice, however, some mass dependence of these profiles is expected. For example, if the concentration of the gas distribution depends on mass similarly to the concentration of the DM profile (Ascasibar et al. 2006), the weak mass dependence of DM concentration implies weak mass dependence of $\tilde{\rho}_{\mathrm{g}}(x)$ and $\tilde{T}(x)$. Indeed, concentration depends on mass even in purely self-similar models (Cole \& Lacey 1996; Navarro, Frenk \& White 1997). These dependencies imply that predictions of the Kaiser model may not describe accurately even the purely self-similar evolution. This is evidenced by deviations of scaling relation evolution from these predictions in hydrodynamical simulations of cluster formation even in the absence of any physical processes that can break self-similarity (e.g., Nagai 2006, Stanek et al. 2010).

In addition, the characteristic gas density, $\rho_{\mathrm{g} *}$, may be mildly modified by a mass-dependent, non self-similar process during some early stage of evolution. If such a process does not 
introduce a pronounced mass scale and is confined to some early epochs (e.g., owing to shutting off of star formation in cluster galaxies due to AGN feedback and gas accretion suppression), then subsequent evolution may still be described well by the self-similar model. The Kaiser model is just the simplest specific example of a more general class of self-similar models, and can therefore be extended to take into account deviations described above.

For simplicity, let us assume that the scalings of gas mass and gas mass fraction against total mass can be expressed as a power law of mass:

$$
M_{\mathrm{g}}=C_{\mathrm{g}} M^{1+\alpha_{\mathrm{g}}}, \quad f_{\mathrm{g}} \equiv \frac{M_{\mathrm{g}}}{M}=C_{\mathrm{g}} M^{\alpha_{\mathrm{g}}}=C_{\mathrm{g}} M_{\mathrm{NL}}^{\alpha_{\mathrm{g}}} \mu^{\alpha_{\mathrm{g}}} .
$$

This does not violate the self-similarity of the problem per se, as long as dimensionless properties of an object, such as $f_{\mathrm{g}}$, remain a function of only the dimensionless mass $\mu(\mathrm{z}) \equiv$ $M / M_{\mathrm{NL}}(\mathrm{z})$. This means that the normalization of the $M_{\mathrm{g}}-M$ relation must scale as $C_{\mathrm{g}} \propto$ $M_{\mathrm{NL}}^{-\alpha_{\mathrm{g}}}$ during the self-similar stages of evolution, such that

$$
M_{\mathrm{g}}=C_{\mathrm{g} 0} M_{\mathrm{NL}}^{-\alpha_{\mathrm{g}}}(\mathrm{z}) M^{1+\alpha_{\mathrm{g}}}=C_{\mathrm{g} 0} M \mu^{\alpha_{\mathrm{g}}} .
$$

Note that self-similarity requires that the slope $\alpha_{\mathrm{g}}$ does not evolve with redshift.

By analogy with the $M_{\mathrm{g}}-M$ relation, we can assume that the $T-M$ relation can be well described by a power law of the form

$$
T=C_{\mathrm{T}} \Delta^{\frac{1}{3}} M^{\frac{2}{3}+\alpha_{\mathrm{T}}},
$$

where $\alpha_{\mathrm{T}}$ describes mild deviation from the scaling due, e.g., to mild dependence of gas and temperature profile slopes in the HE equation. The dimensionless quantities involving temperature $T$ can be constructed using ratios of $T$ with $T_{\mathrm{NL}}=\left(\mu m_{p} / k_{B}\right) G M_{\mathrm{NL}} / R_{\mathrm{NL}} \propto$ $\Delta^{1 / 3} M_{\mathrm{NL}}^{2 / 3}$. As before for the gas fraction, requirement that such dimensionless ratio depends only on $\mu$ requires $C_{\mathrm{T}} \propto M_{\mathrm{NL}}^{-\alpha_{\mathrm{T}}}$ so that

$$
T=C_{\mathrm{To}} M_{\mathrm{NL}}^{-\alpha_{\mathrm{T}}} \Delta^{\frac{1}{3}} M^{\frac{2}{3}+\alpha_{\mathrm{T}}}=C_{\mathrm{To}} \Delta^{\frac{1}{3}} M^{\frac{2}{3}} \mu^{\alpha_{\mathrm{T}}},
$$

Other observable-mass scaling relations can be constructed in the manner similar to the derivation of the original relations above. These are summarized below for the specific choice of $\Delta \rho_{r} \equiv \Delta_{c} \rho_{\mathrm{cr}}(\mathrm{z}) \propto h^{2} E^{2}(\mathrm{z})$ :

$$
\begin{aligned}
M_{\mathrm{g}} & \propto M_{\mathrm{NL}}^{-\alpha_{\mathrm{g}}}(\mathrm{z}) M_{\Delta_{c}}^{1+\alpha_{\mathrm{g}}}, \\
T & \propto E(\mathrm{z})^{2 / 3} M_{\mathrm{NL}}^{-\alpha_{\mathrm{T}}} M_{\Delta_{c}}{ }^{2 / 3+\alpha_{\mathrm{T}}} \\
L_{\mathrm{bol}} & \propto E(\mathrm{z})^{7 / 3} M_{\mathrm{NL}}^{-2 \alpha_{\mathrm{g}}-\alpha_{\mathrm{T}} / 2}(\mathrm{z}) M_{\Delta_{c}}{ }^{4 / 3+2 \alpha_{\mathrm{g}}+\frac{\alpha_{\mathrm{T}}}{2}}, \\
K & \propto E^{-2 / 3}(\mathrm{z}) M_{\mathrm{NL}}^{\frac{2}{3} \alpha_{\mathrm{g}}-\alpha_{\mathrm{T}}}(\mathrm{z}) M_{\Delta_{c}}{ }^{\frac{2}{3}\left(1-\alpha_{\mathrm{g}}\right)+\alpha_{\mathrm{T}}}, \\
Y & \propto E(\mathrm{z})^{2 / 3}(\mathrm{z}) M_{\mathrm{NL}}^{-\alpha_{\mathrm{g}}-\alpha_{\mathrm{T}}}(\mathrm{z}) M_{\Delta_{c}}{ }^{5 / 3+\alpha_{\mathrm{g}}+\alpha_{\mathrm{T}}} .
\end{aligned}
$$

In all of the relations one can, of course, recover the original relations by setting $\alpha_{\mathrm{g}}=\alpha_{\mathrm{T}}=$ 0 . The observable-mass relations can be used to predict observable-observable relations by eliminating mass from the corresponding relations above. 
Note that the evolution of scaling relations in this extended model arises both from the redshift dependence of $\Delta(\mathrm{z}) \rho_{r}(\mathrm{z})$ and from the extra redshift dependence due to factors involving $M_{\mathrm{NL}}$. The practical implication is that if measurements show that $\alpha_{\mathrm{g}} \neq 0$ and/or $\alpha_{\mathrm{T}} \neq 0$ at some redshift, the original Kaiser scaling relations are not expected to describe the evolution, even if the evolution is self-similar. Instead, relations given by Equations 27 31 should be used. Note that at $\mathrm{z} \approx 0$, observations indicate that within the radius $r_{500}$ enclosing overdensity $\Delta_{c}=500, \alpha_{\mathrm{g}} \approx 0.1-0.2$, while $\alpha_{\mathrm{T}} \approx 0 .-0.1$. Therefore, the extra evolution compared to the Kaiser model predictions due to factors involving $\alpha_{\mathrm{g}}$ and, to a lesser degree, factors involving $\alpha_{\mathrm{T}}$ is expected. Such evolution, consistent with predictions of the above equations, is indeed observed both in simulations (see, e.g., Fig. 10 in Vikhlinin et al. 2009b) and in observations (Lin et al. 2012, although see Böhringer, Dolag \& Chon 2012.

In practice, evolution of the scaling relations can be quite a bit more complicated than the evolution predicted by the above equations. The complication is not due to any deviation from self-similarity but rather due to specific mass definition and the fact that cluster formation is an extended process that is not characterized by a single collapse epoch. Some clusters evolve only mildly after their last major merger. However, the mass of such clusters will change with $z$ even if their potential does not change, simply because mass definition is tied to a reference density that changes with expansion of the Universe and because density profiles of clusters extends smoothly well beyond the virial radius. Any observable property of clusters that has radial profile differing from the mass profile, but which is measured within the same $R_{\Delta}$, will change differently than mass with redshift. As a simplistic toy model, consider a population of clusters that does not evolve from $\mathrm{z}=1$ to $\mathrm{z}=0$. Their $\mathrm{X}$-ray luminosity is mostly due to the ICM in the central regions of clusters and it is not sensitive to the outer boundary of integration as long as it is sufficiently large. Thus, X-ray luminosity of such a non-evolving population will not change with z, but masses $M_{\Delta}$ of clusters will increase with decreasing $\mathrm{z}$ as the reference density used to define the cluster boundary decreases. Normalization of the $L_{X}-M_{\Delta}$ relation will thus decrease with decreasing redshift simply due to the definition of mass. The strength of the evolution will be determined by the slope of the mass profile around $R_{\Delta}$, which is weakly dependent on mass. Such an effect may, thus, result in the evolution of both the slope and normalization of the relation. In this respect, quantities that have radial profiles most similar to the total mass profile (e.g., $M_{\mathrm{g}}, Y$ ) will suffer the least from such spurious evolution.

Finally, we note again that in principle for general non power-law initial perturbation spectra of the CDM models the scaling with $M / M_{\mathrm{NL}}$ needs to be replaced with scaling with the peak height $v$. For clusters within a limited mass range, however, the power spectrum can be approximated by a power law and thus a characteristic mass similar to $M_{\mathrm{NL}}$ can be constructed, although such mass should be within the typical mass range of the clusters. The latter is not true for $M_{\mathrm{NL}}$, which is considerably smaller than typical cluster mass at all z.

\subsubsection{Practical implications for observational calibrations of scaling relations.}

In observational calibrations of the cluster scaling relations, it is often necessary to rescale between different redshifts either to bring results from the different $\mathrm{z}$ to a common redshift, or because the scaling relation is evaluated using clusters from a wide range of redshifts due to small sample size. It is customary to use predictions of the Kaiser model to carry out such rescaling to take into account the redshift dependence of $\Delta(\mathrm{z})$. In this context, one 
should keep in mind that these predictions are approximate due to the approximate nature of some of the assumptions of the model, as discussed above. Inaccuracies introduced by such scalings may, for example, then be incorrectly interpreted as intrinsic scatter about the scaling relation.

In addition, because the $\Delta(\mathrm{z})$ factors are a result of an arbitrary mass definition, they should not be interpreted as physically meaningful factors describing evolution of mass. For example, in the $T-M$ relation, the $\Delta^{1 / 3}$ factor in Equation 17 arises due to the dimensional $M / R$ factor of the HE equation. As such, this factor does not change even if the powerlaw index of the $T-M$ relation deviates from $2 / 3$, in which case the relation has the form $T \propto \Delta^{1 / 3} M^{2 / 3+\alpha_{T}}$. In other words, if one fits for the parameters of this relation, such as normalization $A$ and slope $B$, using measurements of temperatures and masses for a sample of clusters spanning a range of redshifts, the proper parameterization of the fit should be

$$
\frac{T}{T_{\mathrm{p}}}=A \Delta^{1 / 3}\left(\frac{M}{M_{\mathrm{p}}}\right)^{B}, \text { or } \frac{T}{T_{\mathrm{p}}}=A \Delta^{1 / 3}\left(\frac{M}{M_{\mathrm{p}}}\right)^{2 / 3+B},
$$

where $T_{\mathrm{p}}$ and $M_{\mathrm{p}}$ are appropriately chosen pivots. The parameterization $T / T_{\mathrm{p}}=A\left(\Delta^{1 / 3} M / M_{\mathrm{p}}\right)^{B}$ that is sometimes adopted in observational analyses is not correct in the context of the selfsimilar model. In other words, only the observable quantities should be rescaled by the $\Delta \rho_{r}$ factors, and not the mass. Likewise, only the $\Delta \rho_{r}$ factors actually predicted by the Kaiser model should be present in the scalings. For example, no such factor is predicted for the $M_{\mathrm{g}}-M$ relation and therefore the gas and total masses of clusters at different redshifts should not be scaled by $\Delta \rho_{r}$ factors in the fits of this relation.

Finally, we note that observational calibrations of the observable-mass scaling relations generally depend on the distances to clusters and are therefore cosmology dependent. Such dependence arises because distances are used to convert observed angular scale to physical scale within which an "observable" is defined, $R=\theta d_{A}(\mathrm{z}) \propto \theta h^{-1}$, or to convert observed flux $f$ to luminosity, $L=4 \pi f d_{L}(\mathrm{z})$, where $d_{A}(\mathrm{z})$ and $d_{L}(\mathrm{z})=d_{A}(\mathrm{z})(1+\mathrm{z})^{2}$ are the angular diameter distance and luminosity distance, respectively. Thus, if the total mass $M$ of a cluster is measured using the HSE equation, we have $M_{\mathrm{HE}} \propto T R \propto d_{A} \propto h^{-1}$. The same scaling is expected for the mass derived from the weak lensing shear profile measurements.

If the gas mass is measured from the X-ray flux from a volume $V \propto R^{3} \propto \theta^{3} d_{A}^{3}$, which scales as $f=L_{\mathrm{X}} /\left(4 \pi d_{L}^{2}\right) \propto \rho_{\mathrm{g}}^{2} V / d_{L}^{2} \propto M_{\mathrm{g}}^{2} /\left(V d_{L}^{2}\right) \propto M_{\mathrm{g}}^{2} /\left(\theta d_{L}^{2} d_{A}^{3}\right)$ and where $f$ and $\theta$ are observables, gas mass then scales with distance as $M_{\mathrm{g}} \propto d_{L} d_{A}^{3 / 2} \propto h^{5 / 2}$. This dependence can be exploited to constrain cosmological parameters, as in the case of X-ray measurements of gas fractions in clusters (Allen et al. 2008, 2004; Ettori et al. 2009; Ettori, Tozzi \& Rosati 2003; LaRoque et al. 2006) or abundance evolution of clusters as a function of their observable (e.g., Vikhlinin et al. 2009c). In this respect, the $M_{\mathrm{g}}-M$ relation has the strongest scaling with distance and cosmology, whereas the scaling of the $T-M$ relation is the weakest (e.g., see discussion by Vikhlinin et al. 2009b).

\subsection{Cluster formation and Thermodynamics of the Intra-cluster gas}

Gravity that drives the collapse of the initial large-scale density peaks affects not only the properties of the cluster DM halos, but also the thermodynamic properties of the intracluster plasma. The latter are also affected by processes related to galaxy formation, such as cooling and feedback. Below, we discuss the thermodynamic properties of the ICM 
resulting from gravitational heating, radiative cooling, and stellar and AGN feedback during cluster formation.

\subsubsection{Gravitational collapse of the intra-cluster gas.}

The diffuse gas infalling onto the DM-dominated potential wells of clusters converts the kinetic energy acquired during the collapse into thermal energy via adiabatic compression and shocks. As gas settles into HE, its temperature approaches values close to the virial temperature corresponding to the cluster mass. In the spherically symmetric collapse model of Bertschinger (1985), supersonic accretion gives rise to the expanding shock at the interface of the inner hydrostatic gas with a cooler, adiabatically compressed, external medium. Real three-dimensional collapse of clusters is more complicated and exhibits large deviations from spherical symmetry, as accretion proceeds both in a quasi-spherical fashion from low-density regions and along relatively narrow filaments. The gas accreting along the latter penetrates much deeper into the cluster virial region and does not undergo a shock at the virial radius (see Fig. 9). The strong shocks are driven not just by the accretion of gas from the outside but also "inside-out" during major mergers (e.g., Poole et al. 2007).

The shocks arising during cluster formation can be classified into two broad categories: strong external shocks surrounding filaments and the virialized regions of DM halos and weaker internal shocks, located within the cluster virial radius (e.g., Pfrommer et al. 2006, Skillman et al. 2008, Vazza et al. 2009). The strong shocks arise in the high-Mach number flows of the intergalactic gas, whereas weak shocks arise in the relatively low-Mach number flows of gas in filaments and accreting groups, which was pre-heated at earlier epochs by the strong shocks surrounding filaments or external groups. The left panel of Figure 9 shows these two types of shocks in a map of the shocked cells identified in a cosmological adaptive mesh refinement simulation of a region surrounding a galaxy cluster (from Vazza et al. 2009), along with the gas velocity field. This map highlights the strong external shocks, characterized by high Mach numbers $\mathcal{M}>30$, surrounding the cluster at several virial radii from the cluster center, and weaker internal shocks, with $\mathcal{M} \lesssim 2-3$. The cluster is shown at the epoch immediately following a major merger, which generated substantial velocities of gas within virial radius. As we discuss in $\S 4$ below, incomplete thermalization of these gas motions is one of the main sources of non-thermal pressure support in the ICM.

The right panel of Figure 9 shows the distribution of the kinetic energy processed by shocks, as a function of the local shock Mach number, for different redshifts (Skillman et al. 2008). The figure shows that a large fraction of the kinetic energy is processed by weak internal shocks and this fraction increases with decreasing redshift as more and more of the accreting gas is pre-heated in filaments. Yet, the left panel of Fig. 9 highlights that large- $\mathcal{M}$ shocks surround virialized halos in such a way that most gas particles accreted in a galaxy cluster must have experienced at least one strong shock in their past.

Becasuse gravity does not have a characteristic length scale, we expect the predictions of the self-similar model, presented in Section 3.9, to apply when gravitational gas accretion determines the thermal properties of the ICM. The scaling relations and their evolution predicted by the self-similar model are indeed broadly confirmed by the non-radiative hydrodynamical simulations that include only gravitational heating (e.g., Eke, Navarro \& Frenk 1998; Nagai, Kravtsov \& Vikhlinin 2007; Navarro, Frenk \& White 1995), although some small deviations arising due to small differences in the dynamics of baryons and DM were also found (Ascasibar et al. 2006, Nagai 2006, Stanek et al. 2010). 

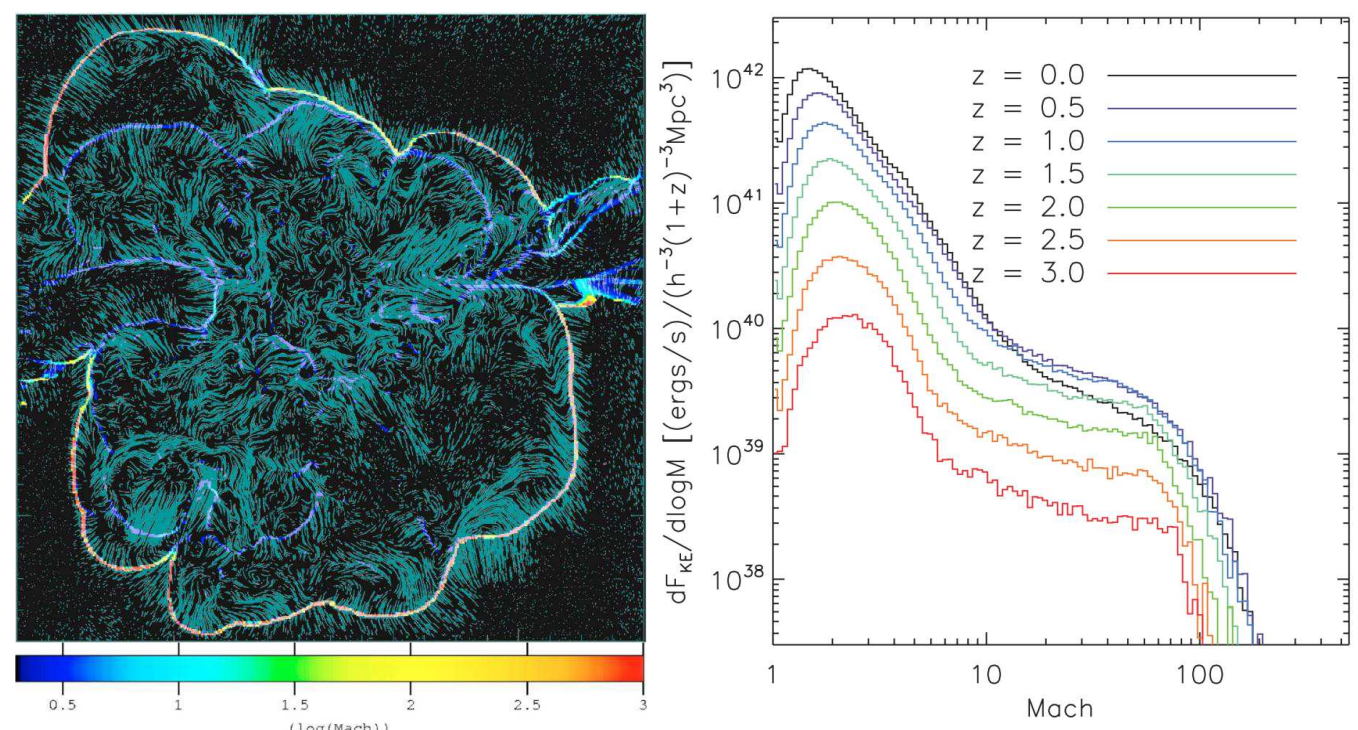

Fig. 9. Left panel: map of the shocked cells identified by the divergence of velocity colored by the local shock Mach number and turbulent gas velocity eld (streamlines) in a slice of the simulation box $7.5 \mathrm{Mpc}$ on a side and depth of $18 \mathrm{kpc}$ at $\mathrm{z}=0.6$, for a simulated cluster that reaches a mass of $\sim 2 \times 10^{14} M_{\odot}$ by z $=0$ (from Vazza et al. 2009). Right panel: redshift evolution of the distribution of the kinetic energy processed by shocks, as a function of the Mack number $\mathcal{M}$ in a cosmological simulation (from Skillman et al. 2008). Results shown in both panels are based on the adaptive mesh refinement ENZO code (O'Shea et al. 2004).

As discussed in Section 2, observations carried out with the Chandra and XMM-Newton telescopes during the past decade showed that the outer regions of clusters $\left(r \gtrsim r_{2500}\right)$ exhibit self-similar scaling, whereas the core regions exhibit strong deviations from selfsimilarity. In particular, gas density in the core regions of small-mass clusters is lower than expected from self-similar scaling of large-mass systems. These results indicate that some additional non-gravitational processes are shaping properties of the ICM. We review some of these processes studied in cluster formation models below.

\subsubsection{Phenomenological pre-heating models.}

The first proposed mechanism to break self-similarity was high-redshift $\left(\mathrm{z}_{h} \geq 3\right)$ pre-heating by non-gravitational sources of energy, presumably by a combined action of the AGN and stellar feedback (Evrard \& Henry 1991, Kaiser 1991). The specific extra heating energy per unit mass, $E_{h}$, defines the temperature scale $T^{*} \propto E_{h} / k_{B}$, such that clusters with virial temperature $T_{\mathrm{vir}}>T^{*}$ should be left almost unaffected by the extra heating, whereas in smaller clusters with $T_{\text {vir }}<T^{*}$ gas accretion is suppressed. As a result, gas density is relatively lower in lower massive systems, especially at smaller radii, while their entropy will be higher. 
Both analytical models (e.g. Babul et al. 2002, Tozzi \& Norman 2001, Voit et al. 2003) and hydrodynamical simulations (e.g. Bialek, Evrard \& Mohr 2001; Borgani et al. 2002; Muanwong et al. 2002) have demonstrated that with a suitable pre-heating prescription and typical heating injection of $E_{h} \sim 0.5-1 \mathrm{keV}$ per gas particle self-similarity can be broken to the degree required to reproduce observed scaling relations. Studies of the possible feedback mechanisms show that such amounts of energy cannot be provided by SNe (e.g., Borgani et al. 2004, Henning et al. 2009, Kay et al. 2007, Kravtsov \& Yepes 2000, Renzini 2000), and must be injected by the AGN population (e.g., Bower, McCarthy \& Benson 2008; Lapi, Cavaliere \& Menci 2005; Wu, Fabian \& Nulsen 2000) or by some other unknown source.

However, regardless of the actual sources of heating, strong widespread heating at high redshifts would conflict with the observed statistical properties of the Lyman- $\alpha$ forest (Borgani \& Viel 2009; Shang, Crotts \& Haiman 2007). Moreover, hydrodynamical simulations have demonstrated that simple pre-heating models predict large isentropic cores (e.g., Borgani et al. 2005, Younger \& Bryan 2007) and shallow pressure profiles (Kay et al. 2012). This is at odds with the entropy and pressure profiles of real clusters which exhibit smoothly declining entropy down to $r \sim 10-20 \mathrm{kpc}$ (e.g., Arnaud et al. 2010, Cavagnolo et al. 2009).

\subsubsection{The role of radiative cooling.}

The presence of galaxies in clusters and low levels of the ICM entropy in cluster cores are a testament that radiative cooling has operated during cluster formation in the past and is an important process shaping thermodynamics of the core gas at present. Therefore, in general radiative cooling cannot be neglected in realistic models of cluster formation. Given that cooling generally introduces new scales, it can break self-similarity of the ICM even in the absence of heating (Voit \& Bryan 2001). In particular, cooling removes low-entropy gas from the hot ICM phase in the cluster cores, which is replaced by higher entropy gas from larger radii. Somewhat paradoxically, the cooling thus leads to an entropy increase of the hot, X-ray emitting ICM phase. This effect is illustrated in Figure 10, which shows the entropy maps in the simulations of the same cluster with and without cooling. In the absence of cooling (left panel), the innermost region of the cluster is filled by low-entropy gas. Merging substructures also carry low-entropy gas, which generates comet-like features by ram-pressure stripping, and is hardly mixed in the hotter ambient of the main halo. In the simulation with radiative cooling (right panel), most of the low-entropy gas associated with substructures and the central cluster region is absent, and most of the ICM has a relatively high entropy.

A more quantitative analysis of the entropy distribution for these simulated clusters is shown in Figure 11, in which the entropy profiles of clusters simulated with inclusion of different physical processes are compared with the baseline analytic spherical accretion model; this model predicts the power-law entropy profile $K(r) \propto r^{1.1}$ (e.g. Tozzi \& Norman 2001, Voit 2005). The figure shows that the entropy profile in the simulation with radiative cooling is significantly higher than that of the non-radiative simulation. The difference in entropy is as large as an order of magnitude in the inner regions of the cluster and is greater by a factor of two even at $r_{500}$.

Interestingly, the predicted level of entropy at $r \sim r_{2500}-r_{500}$ in the simulations with cooling (but no significant heating) is consistent with the ICM entropy inferred from X- 

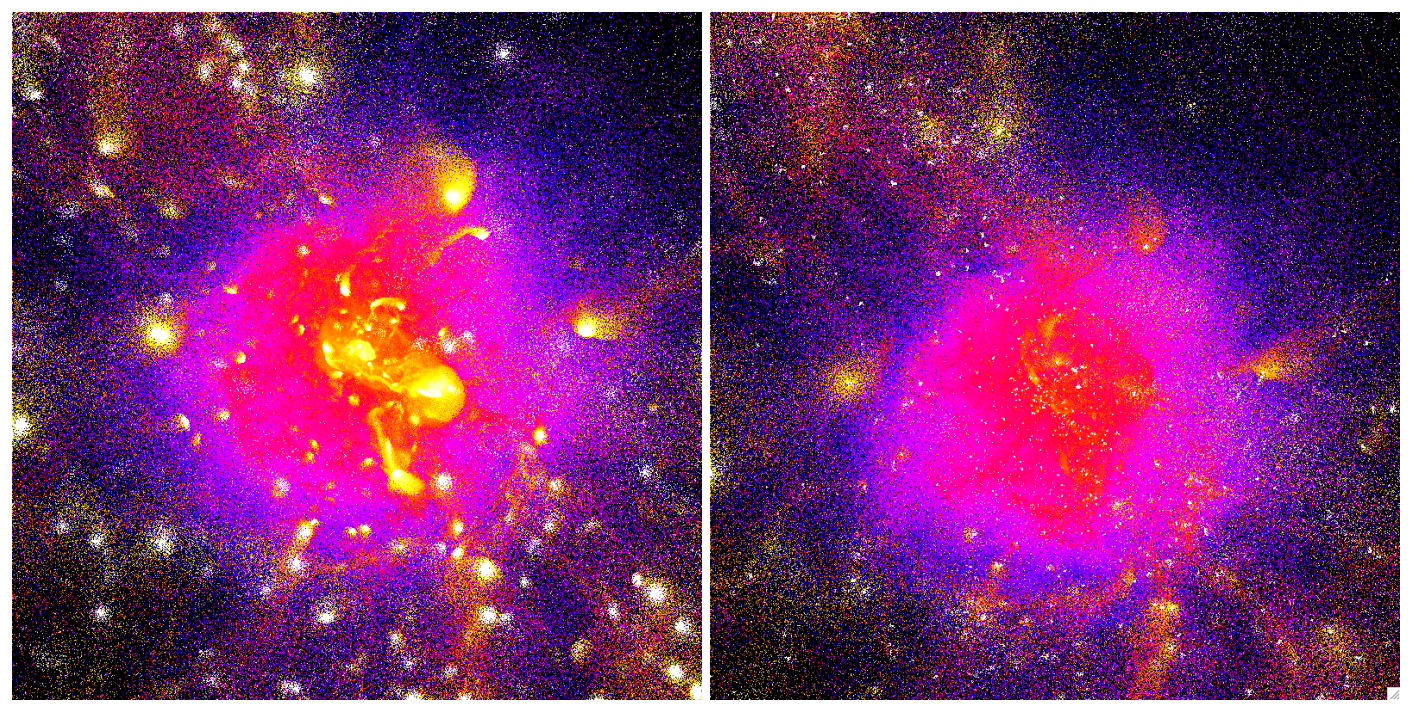

Fig. 10. Maps of entropy in cosmological hydrodynamical simulations of a galaxy cluster of mass $M_{500} \simeq 10^{15} h^{-1} M_{\odot}$ at $\mathrm{z}=0$, carried out without (left panel) and with (right panel) radiative cooling. Brighter colors correspond to lower gas entropy. Each panel encompasses a physical scale of $6.5 h^{-1} \mathrm{Mpc}$, which corresponds to $\approx 2.5$ virial radii for this cluster. The simulations have been carried out using the GADGET-3 smoothed particle hydrodynamics code Springel (2005).

ray observations. However, this agreement is likely to be spurious because it is achieved with the amount of cooling that results in conversion of $\approx 40 \%$ of the baryon mass in clusters into stars and cold gas, which is inconsistent with observational measurements of cold fraction varying from $\simeq 20-30 \%$ for small-mass, X-ray-emitting clusters to $\lesssim 10 \%$ for massive clusters (see $\S 2$ ).

Finally, note that inclusion of cooling in simulations with pre-heating discussed above usually results in problematic star-formation histories. In fact, if pre-heating takes place at a sufficiently high redshift, clusters exhibit excessive cooling at lower redshifts, as pre-heated gas collapses and cools at later epochs compared to the simulations without pre-heating (e.g. Tornatore et al. 2003). These results highlight the necessity to treat cooling and heating processes simultaneously using heating prescriptions that can realistically reproduce the heating rate of the ICM gas as a function of cosmic time. We discuss efforts in this direction next.

\subsubsection{Thermodynamics of the intracluster medium with stellar and active galactic nuclei feedback.}

The results discussed above strongly indicate that, in order to reproduce the overall properties of clusters, cooling should be modelled together with a realistic prescription for nongravitational heating. This is particularly apparent in the cluster cores, where a steady heating is required to offset the ongoing radiative cooling observed in the form of strong X-ray emission (see, e.g., Peterson \& Fabian 2006). Studies of the feedback processes in clusters 


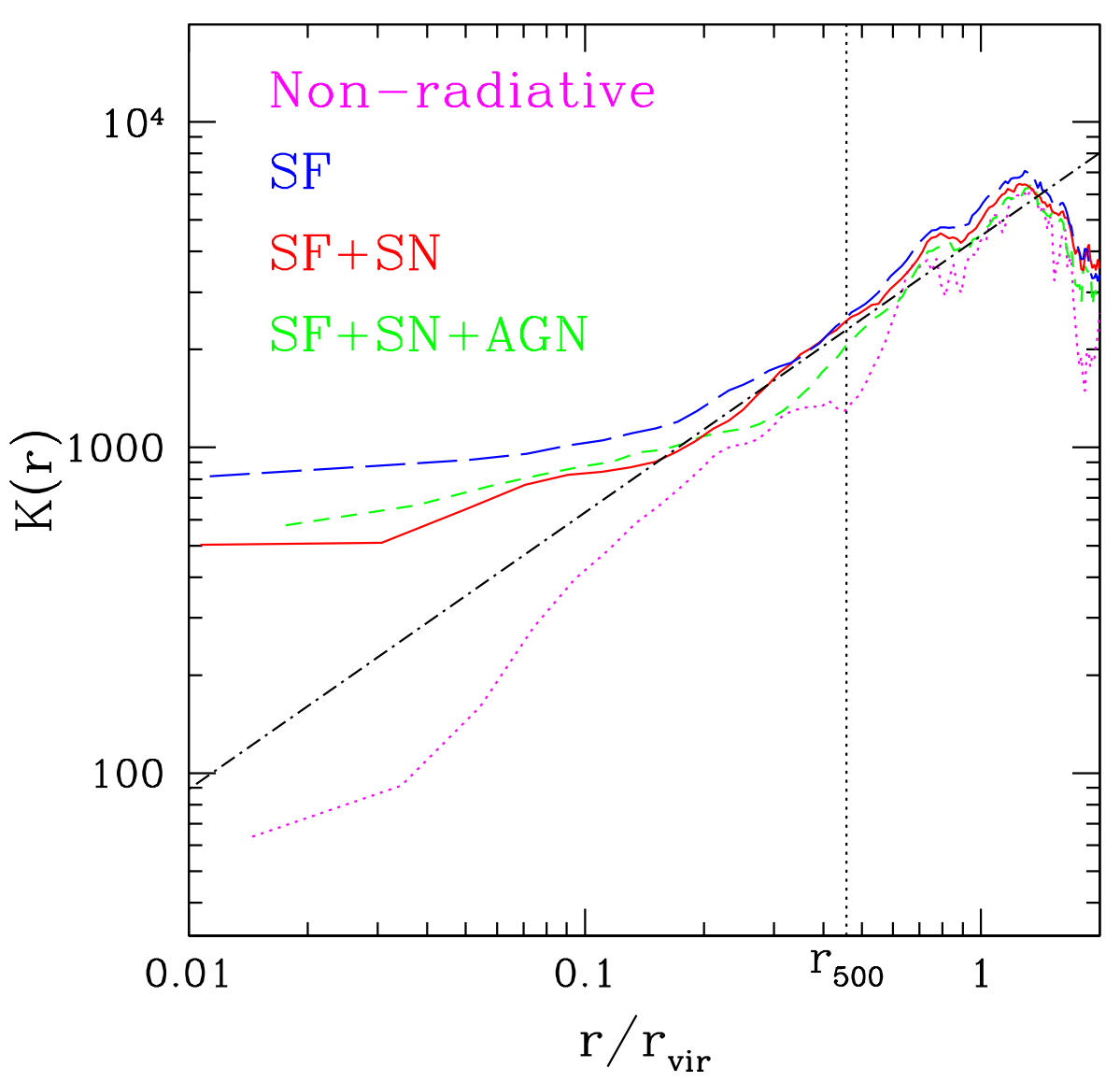

Fig. 11. Radial profiles of entropy (in units of kiloelectronvolt-centimeters squared) for the same simulations whose entropy maps are shown in Figure 10. Magenta dotted, long-dashed blue, continuous red, and short-dashed green curves refer to the non-radiative simulation and to the three radiative simulations including only cooling and star formation, including also the effect of galactic ejecta from supernova, and including also the effect of AGN feedback, respectively. The dot-dashed line shows the power-law entropy profile with slope $K(r) \propto r^{1.1}$, whereas the vertical dotted line marks the position of $r_{500}$.

is one of the frontiers in cluster formation modelling. Although we do not yet have a complete picture of the ICM heating, a number of interesting and promising results have been obtained.

In Figure 11, the solid line shows the effect of the SN feedback on the entropy profile. In these simulations, the kinetic feedback of SNe is included in the form of galactic winds carrying the kinetic energy comparable to all of the energy released by Type-II SNe expected to occur according to star formation in the simulation. This energy partially compensates for the radiative losses in the central regions, which leads to a lower level of entropy in the core. However, the core ICM entropy in these simulations is still considerably higher than observed (e.g., Sun et al. 2009). The inefficiency of the SN feedback in offsetting the cooling sufficiently is also evidenced by temperature profiles. 
Figure 4 (from Leccardi \& Molendi 2008) compares the observed temperature profiles of a sample of local clusters with results from simulations that include the SN feedback. The figure shows that simulations reproduce the observed temperature profile at $r \approx 0.2 r_{180}$. The overall shape of the profile at these large radii is reproduced by simulations including a wide range of physical processes, including non-radiative simulations (e.g., Borgani et al. 2004; Loken et al. 2002; Nagai, Kravtsov \& Vikhlinin 2007). At large radii, however, the observed and predicted profiles do not match: The profiles in simulated clusters continue to increase to the smallest resolved radii, whereas the observed profiles reach a maximum temperature $T_{\max } \approx 2 T_{180}$ and then decrease with decreasing radius to temperatures of $\sim 0.1-0.3 T_{\max }$. The high temperatures of the central gas reflects its high entropy and is due to the processes affecting the entropy, as discussed above.

Another indication that the $\mathrm{SN}$ feedback alone is insufficient is the fact that the stellar mass of the BCGs in simulations that include only the feedback from $\mathrm{SNe}$ is a factor of two to three larger than the observed stellar masses. For example, in the simulated clusters shown in figure 10 , the baryon fraction in stars within $r_{500}$ decreases from $\simeq 40 \%$ in simulations without $\mathrm{SN}$ feedback to $\simeq 30 \%$, which is still a factor of two larger than observational measurements. The overestimate of the stellar mass is reflected in the overestimate of the ICM metallicity in cluster cores (e.g., Borgani et al. 2008, and references therein).

Different lines of evidence indicate that energy input from the AGN in the central cluster galaxies can provide most of the energy required to offset cooling (see McNamara \& Nulsen 2007 , for a comprehensive review). Because the spatial and temporal scales resolved in cosmological simulations are larger than those relevant for gas accretion and energy input, the AGN energy feedback can only be included via a phenomenological prescription. Such prescriptions generally model the feedback energy input rate by assuming the Bondi gas accretion rate onto the SMBHs, included as the sink particles, and incorporate a number of phenomenological parameters, such as the radiative efficiency and the feedback efficiency, which quantify the fraction of the radiated energy that thermally couples to the surrounding gas (e.g., Springel, Di Matteo \& Hernquist 2005). The values of these parameters are adjusted so that simulations reproduce the observed relation between black hole mass and the velocity dispersion of the host stellar bulge (e.g., Marconi \& Hunt 2003). An alternative way of implementing the AGN energy injection is the AGN-driven winds, which shock and heat the surrounding gas (e.g., Dubois et al. 2011, Gaspari et al. 2011, Omma et al. 2004).

In general, simulations of galaxy clusters based on different variants of these models have shown that the AGN feedback can reduce star formation in massive cluster galaxies and reduce the hot gas content in the poor clusters and groups, thereby improving agreement with the observed relation between X-ray luminosity and temperature (e.g., Puchwein, Sijacki \& Springel 2008; Sijacki et al. 2007). Figure 12 (from Martizzi, Teyssier \& Moore 2012) shows that simulations with the AGN feedback results in stellar masses of the BCGs that agree with the masses required to match observed stellar masses of galaxies and masses of their DM halos predicted by the models. The figure also shows that stellar masses are overpredicted in the simulations without the AGN feedback. Incidentally, the large-scale winds at high redshifts and stirring of the ICM in cluster cores by the AGN feedback also help to bring the metallicity profiles into cluster simulations in better agreement with observations (Fabjan et al. 2010, McCarthy et al. 2010).

Although results of simulations with the AGN feedback are promising, simulations so far have not been able to convincingly reproduce the observed thermal structure of cool cores. As an example, Figure 11 shows that the entropy profiles in such simulations still develop 


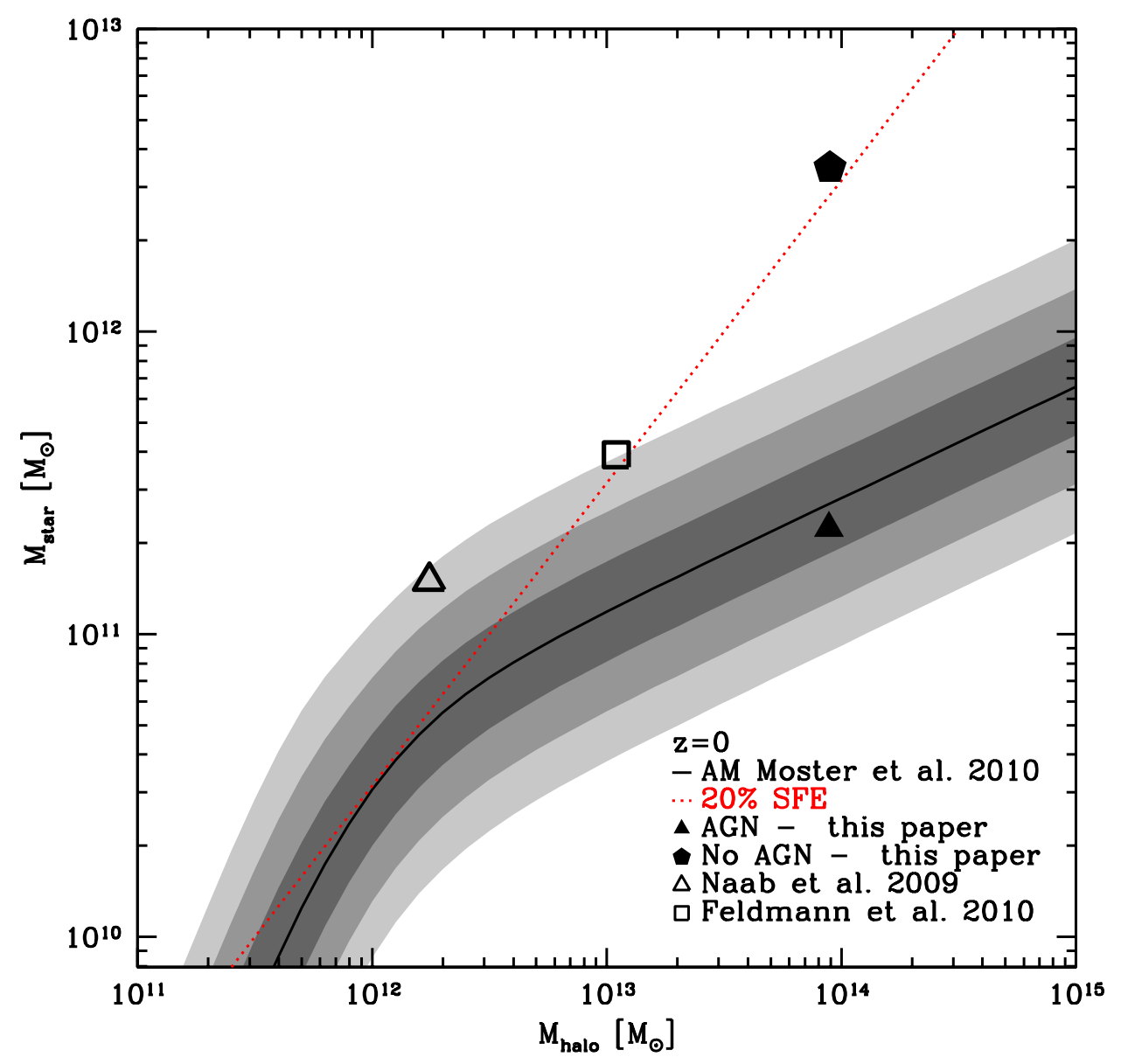

Fig. 12. Comparison of the relation between stellar mass and total halo mass as predicted by cosmological hydrodynamical simulations of four early-type galaxies (symbols) (from Martizzi, Teyssier \& Moore 2012). The open triangle and square refer to the simulations presented by Naab, Johansson \& Ostriker (2009) and by Feldmann et al. (2010), both based on the smoothed particle hydrodynamics codes and not including AGN feedback. The filled symbols refer to the simulations by Martizzi, Teyssier \& Moore (2012) with the brightest cluster galaxies forming at the center of a relatively poor cluster carried out with an AMR code, both including (triangle) and excluding (pentagon) AGN feedback. The red dotted line represents the relation expected for $20 \%$ efficiency in the conversion of baryons into stars. The solid black line is the prediction from Moster et al. (2010) of a model in which dark matter halos are populated with stars in such a way as to reproduce the observed stellar mass function. The grey shaded areas represent the 1-, 2- and 3- $\sigma$ scatter around the average relation.

large constant entropy core inconsistent with observed profiles. Interestingly, the adaptive mesh refinement simulations with jet-driven AGN feedback by Dubois et al. (2011) reproduce the monotonically decreasing entropy profiles inferred from observations. However, such agreement only exists if radiative cooling does not account for the metallicity of the ICM; in simulations that take into account the metallicity dependence of the cooling rates the entropy profile still develosp a large constant entropy core.

The presence of a population of relativistic particles in AGN-driven high-entropy bubbles has been suggested as a possible solution to this problem Guo \& Oh 2008, Sijacki et al. 
2008). A relativistic plasma increases the pressure support available at a fixed temperature and can, therefore, help to reproduce the observed temperature and entropy profiles in core regions. However, it remains to be seen whether the required population of the cosmic rays is consistent with available constraints inferred from $\gamma$ and radio observations of clusters (e.g., Brunetti 2011, and references therein). A number of additional processes, such as thermal conduction (e.g., Narayan \& Medvedev 2001) or dynamical friction heating by galaxies (El-Zant, Kim \& Kamionkowski 2004) have been proposed. Generally, these processes cannot effectively regulate cooling in clusters by themselves (e.g., Conroy \& Ostriker 2008, Dolag et al. 2004), but they may play an important role when operating in concert with the AGN feedback (Voit 2011) or instabilities in plasma (e.g., Sharma et al. 2012).

In summary, results of the theoretical studies discussed above indicate that the AGN energy feedback is the most likely energy source regulating the stellar masses of cluster galaxies throughout their evolution and suppressing cooling in cluster cores at low redshifts. The latter likely requires an interplay between the AGN feedback and a number of other physical processes: e.g., injection of the cosmic rays in the high-entropy bubbles, buoyancy of these bubbles stabilized by magnetic fields, dissipation of their mechanical energy through turbulence, thermal conduction, and thermal instabilities. Although details of the interplay are not yet understood, it is clear that it must result in a robust self-regulating feedback cycle in which cooling immediately leads to the AGN activity that suppresses further cooling for a certain period of time.

\section{Regularity of the cluster populations}

Processes operating during cluster formation and evolution discussed in the previous section are complex and nonlinear. However, it is now also clear that most of the complexity is confined to cluster cores and affects a small fraction of volume and mass of the clusters. In this regime, clusters' observational properties exhibit strong deviations from the self-similar scalings described in Section 3.9 (see also Voit 2005). At larger radii, ICM is remarkably regular. In this section, we discuss the origins of such highly regular behaviour and the range of radii where it can be expected. We argue that the existence of this radial range allows us to define integral observational quantities, which have low scatter for clusters of a given mass that are not sensitive to the astrophysical processes operating during cluster formation and evolution. This fact is especially important for the current and future uses of clusters as cosmological probes (Allen, Evrard \& Mantz 2011, Weinberg et al. 2012).

\subsection{Characterizing regularity}

A number of observational evidences, based on X-ray measurements of gas density (e.g. Croston et al. 2008) and temperature profiles (Leccardi \& Molendi 2008, Pratt et al. 2007, Vikhlinin et al. 2006), and the combination of the two in the form of entropy profile (Cavagnolo et al. 2009), demonstrate that clusters have a variety of behaviors in central regions, depending on the presence and prominence of cool cores. As discussed in Section 2, outside of core regions, clusters behave as a more homogeneous population and obey assumptions and expectations of the self-similar model (discussed above in 3.9.1). For instance, Figure 3 shows that the ICM density is nearly independent of temperature once measured outside 
of core regions $r \gtrsim r_{2500}$, at least for relatively hot systems with $T \gtrsim 3 \mathrm{keV}$. Quite remarkably, observed and simulated temperature profiles agree with each other within this same radial range (see Figure 4).

A good illustration of the regularity of the ICM properties is represented by the pressure profiles shown in Figure 13 (from Arnaud et al. 2010, but see also Sun et al. 2011) rescaled to the values of radius and pressure at $r_{500}$. The perfectly regular, self-similar behavior would correspond to a single line in this plot for clusters of all masses. The pressure profiles shown in this figure are derived from X-ray observations and are defined as the product of electron number density and temperature profiles. Similar profiles are now derived from SZ observations, which probe pressure more directly (e.g., Bonamente et al. 2012). Quite remarkably, the observed pressure profiles at $r \gtrsim 0.2 r_{500}$ follow a nearly universal profile (see also Nagai, Kravtsov \& Vikhlinin 2007), exhibiting fractional scatter of $\lesssim 30 \%$ at $r \sim 0.2 r_{500}$ and even smaller scatter of $\sim 10-15 \%$ at $r \sim 0.5 r_{500}$. At smaller radii the scatter of pressure profiles is much larger, with steep profiles corresponding to the cool core clusters and flatter profiles for disturbed clusters. Figure 13 shows that simulated and observed pressure profiles agree well with each other for $r \gtrsim 0.2 r_{500}$, i.e., in the regime where the cluster population has a more regular behaviour. At smaller radii the profiles from simulations are on average steeper than observed, and exhibit a lower degree of diversity between cool core and non-cool core clusters.

The scatter in the cluster radial profiles can be used to define the following three radial regimes.

1. Cluster cores, $r \lesssim r_{2500}$, which exhibit the largest scatter and where scaling with mass differs significantly from the self-similar scaling expectation. We do not yet have a complete and adequate theoretical understanding of the observed properties of the ICM and their diversity in the cluster cores. This is one of the areas of active ongoing theoretical and observational research.

2. Intermediate radii, $r_{2500} \lesssim r \lesssim r_{500}$, which exhibit the smallest scatter and scaling with mass close to the self-similar scaling. Although the processes affecting thermodynamics of these regions are not yet fully understood, the simple scaling and regular behavior make observable properties of clusters at these radii useful for connecting them to the total cluster mass.

3. Cluster outskirts $r>r_{500}$, where scatter is increasing with radius and scaling with mass can be expected to be close to self-similar on theoretical grounds, but have not yet been constrained observationally. In this regime, clusters are dynamically younger, characterized by recent mergers, departures from equilibrium, and a significant degree of gas clumping. Significant progress is expected in the near future due to a combination of high-sensitivity SZ and X-ray observations using the next generation of instruments.

The physical origin of the regular scaling with mass is the fact that cluster mass is the key control variable of cluster formation, which sets the amount of gas mass, the average temperature of the ICM, etc. It is important to note that the close to self-similar scaling with mass outside the cluster core does not imply that the non-gravitational physical processes are negligible in this regime. For instance, Sun et al. (2009) showed that entropy measured at $r_{500}$ has a scaling with temperature quite close to the self-similar prediction, yet its level is higher than expected from a simple model in which only gravity determines the evolution of the intra-cluster baryons. This implies that whatever mechanism one invokes to account for such an entropy excess, it must operate in such a way as to not violate the self-similar scaling. The scatter around the mean profile exhibited by clusters at different 
radii can be due to a number of factors. In particular, the small scatter at intermediate radii is a non-trivial fact, given that different clusters of the same mass are in different stages of their dynamical evolution and physical processes affecting their profiles may have operated differently due to different formation histories.

One of the interesting implications of the small scatter in the pressure profiles is that it provides an upper limit on the contribution of non-thermal pressure support or, at least, on its cluster-by-cluster variation. A well-known source of non-thermal pressure is represented by residual gas motions induced by mergers, galaxy motions, and gas inflow along large-scale filaments. Cosmological hydrodynamical simulations of cluster formation have been extensively used to quantify the pressure support contributed by gas motions and the corresponding level of violation of HE (e.g., Ameglio et al. 2009; Biffi, Dolag \& Böhringer 2011; Lau, Kravtsov \& Nagai 2009; Nagai, Kravtsov \& Vikhlinin 2007; Piffaretti \& Valdarnini 2008; Rasia, Tormen \& Moscardini 2004). All these analyses consistently found that ICM velocity fields contribute a pressure support of about $5 \%$ to $15 \%$ per cent of the thermal one, the exact amount depending on the radial range considered (being larger at larger radii) and on the dynamical state of the clusters. Currently, there are only indirect indications for turbulent motions in the ICM of the real clusters from fluctuations of gas density measured in X-ray observations (e.g., Churazov et al. 2012, Schuecker et al. 2004). Direct measurements or upper limits on gas velocities and characterization of their statistical properties should be feasible with future high-resolution spectroscopic and polarimetric instruments on the next-generation X-ray telescopes (e.g., Inogamov \& Sunyaev 2003, Zhuravleva et al. 2010).

The galaxies and groups orbiting or infalling onto clusters not only stir the gas, but also make the ICM clumpier. The dense inner regions of clusters ram-pressure strip the gas on a fairly short time scale, so that the clumping is fairly small near cluster cores. However, it is substantial in the outskirts in cluster simulations where orbital times are longer and accretion of new galaxies and groups is ongoing. Given that the X-ray emissivity scales as the square of the local gas density, the clumpiness can bias the measurement of gas density from X-ray surface brightness profiles toward higher values if it is not accounted for. Because clumping is expected to increase with increasing cluster-centric radius, the inferred slope of gas density profiles can be underestimated, thus affecting the resulting pressure profile and hydrostatic mass estimates. Furthermore, gas clumping also affects $\mathrm{X}-$ ray temperature which is measured by fitting the $\mathrm{X}$-ray spectrum to a single-temperature plasma model (Mazzotta et al. 2004, Vikhlinin 2006). Clumping can therefore contribute to the scatter of pressure profiles at large radii, especially at $r>r_{500}$ (e.g., Nagai \& Lau 2011).

Indirect detections of gas clumping through X-ray observations out to $r_{200}$ have been recently claimed, based on Suzaku observations of a flattening in the X-ray surface brightness profiles at such large radii (Simionescu et al. 2011). However, these results are prone to significant systematic uncertainties (Ettori \& Molendi 2011). Independent analyses based on the ROSAT data (e.g. Eckert et al. 2011)) show the surface brightness profiles steepens beyond $r_{500}$ (see also Neumann 2005; Vikhlinin, Forman \& Jones 1999), which is inconsistent with the degree of gas clumping inferred from the Suzaku data, but consistent with predictions of hydrodynamical simulations.

Clearly, the clumpiness of the ICM depends on a number of uncertain physical processes, such as efficient feedback, which removes gas from merging structures, or thermal conduction, which homogenizes the ICM temperatures (e.g., Dolag et al. 2004). The degree of 


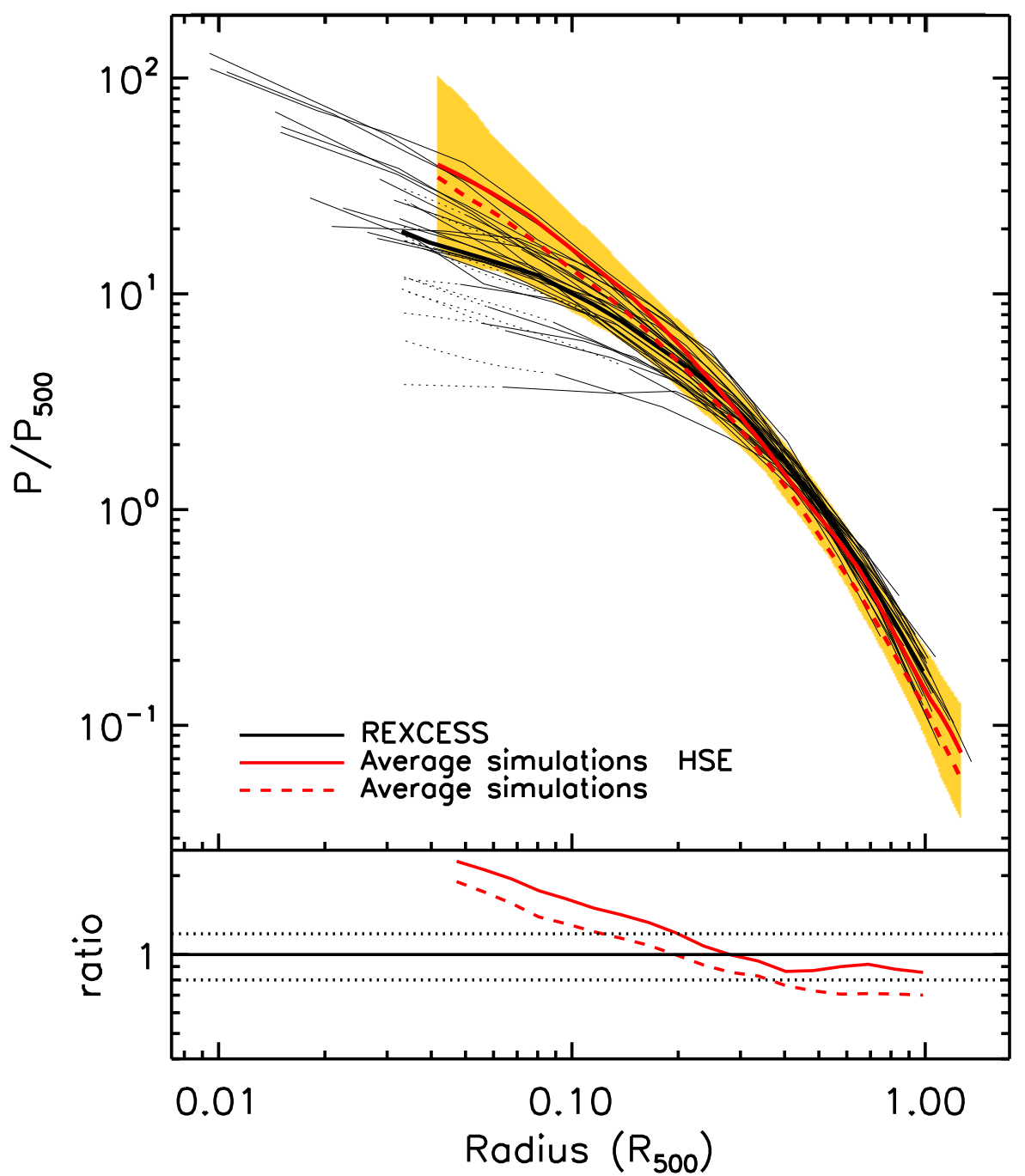

Fig. 13. Comparison between observed (black lines) and simulated (red lines with orange shaded area) pressure profiles (from Arnaud et al. 2010). Observational data refer to the Rrepresentative XMM-Newton Cluster Structure Survey (REXCESS) sample of nearby clusters (Böhringer et al. $2007)$ observed with XMM-Newton. Simulation results are obtained by combining different sets of clusters simulated with both smoothed particle hydrodynamics and adaptive mesh refinement codes (see Arnaud et al. 2010 for details). The continuous red line corresponds to the average profile from simulations, after rescaling profiles according to the values of $R_{500}$ and $M_{500}$ predicted by hydrostatic equilibrium, with the orange area showing the corresponding rms scatter. The red dotted line shows the simulation results when using instead the true $M_{500}$ value. The bottom panel shows the ratio between average simulation profiles and average observed profiles. 
gas clumping in density and temperature is therefore currently uncertain in both theoretical models and observations. Future high-sensitivity SZ observations of galaxy clusters with improved angular resolution will allow a direct measurement of projected pressure profiles. Their comparison with X-ray derived profiles will help in understanding the impact of gas clumping on the thermal complexity of the ICM.

Additional non-thermal pressure support can be provided by the magnetic fields and relativistic cosmic rays, the presence of which in the ICM is demonstrated by radio observations of the radio halos: diffuse and faint radio sources filling the central $\mathrm{Mpc}^{3}$ region of many galaxy clusters (e.g., Giovannini et al. 2009, Venturi et al. 2008) arising due to the synchrotron emission of highly relativistic electrons moving in the ICM magnetic fields. The origin of these relativistic particles still needs to be understood, although several models have been proposed. Shocks and turbulence associated with merger events are expected to compress and amplify magnetic fields and accelerate relativistic electrons (see, e.g., Ferrari et al. 2008 and Dolag, Bykov \& Diaferio 2008 for reviews). Numerical simulations including injection of cosmic rays from accretion shocks and SN explosions (e.g., Pfrommer et al. 2007, Vazza et al. 2012) indicate that cosmic rays contribute a pressure support, which can be as high as $\sim 10 \%$ for relaxed clusters and $\sim 20 \%$ for unrelaxed clusters at the outskirts. At smaller radii, the pressure contribution of cosmic rays in these models becomes small ( $\lesssim 3 \%$ at $r \lesssim 0.1 r_{\text {vir }}$ ), which is consistent with the upper limits from $\gamma$-ray observations by the Fermi Gamma-ray Telescope (e.g., Ackermann et al. 2010).

The role of intracluster magnetic fields have been investigated in a number of studies using cosmological simulations (see Dolag, Bykov \& Diaferio 2008, for a review). The general result is that pressure support from magnetic fields should be limited to $\lesssim 5 \%$, which is consistent with observational constraints on the magnetic field strength $(\sim \mu G)$ (e.g., Govoni et al. 2010, Vogt \& Enßlin 2005) and upper limits on the contribution of magnetic fields to non-thermal pressure support (e.g. Laganá, de Souza \& Keller 2010).

As a summary, the scatter in cluster profiles in the cluster cores is mainly driven by differences in the physical processes such as cooling and heating by AGN feedback and different merger activity that different clusters experienced during their evolution. At intermediate radii, the scatter is small because the ICM is generally in good HE within cluster gravitational potential and because processes that shaped its thermodynamic processes have not introduced new mass scale so that self-similar scaling is not broken. In the cluster outskirts, the scatter is expected to be driven by deviations from HE and other sources of non-thermal pressure support such as the cosmic rays, as well as by a rapid increase of ICM clumpiness with increasing radius.

\subsection{Scaling relations}

Existence of the radial range, where ICM properties scale with mass similarly to the selfsimilar expectation with a small scatter, implies that we can define integral observable quantities within this range that will obey tight scaling relations among themselves and with the total cluster mass. Furthermore, these scaling relations are also expected to be weakly sensitive to the cluster dynamical state, given that relaxed and unrelaxed clusters have similar profiles at these intermediate radii. Indeed, as we showed in $§ 2$ (see Fig. 5), Xray luminosity measured within the radial range $[0.15-1] r_{500}$ exhibits a tight scaling against the total ICM thermal content measured by the $Y_{X}$ parameter, with relaxed and unrelaxed clusters following the same relation. Here $Y_{X}$ is defined as the product of gas mass and 
$\mathrm{X}$-ray temperature, both measured within $r_{500}$ but like $L_{\mathrm{X}}$ temperature is measured after excising the contribution from $r<0.15 r_{500}$.

As discussed in $\S 3.9$, the gas temperature $T$, gas mass $M_{\text {gas }}$ and total thermal content of the ICM $Y=M_{\text {gas }} T$, are commonly used examples of integral observational quantities whose scaling relations with cluster mass are predicted by the self-similar model and for which calibrations based on X-ray and SZ observations (or their combinations) and simulations are available. For example, Figure 14 shows the scaling relation between $Y_{X}$, and $M_{500}$ for simulated clusters and for a set of clusters with detailed Chandra observations from a study by Kravtsov, Vikhlinin \& Nagai (2006), where $Y_{\mathrm{X}}$ was introduced and defined specifically to use the temperature estimated only at $0.15 r_{500}<r<r_{500}$ in order to minimize the scatter. The relation of $Y_{\mathrm{X}}$ with $M_{500}$ in simulations has scatter of only $\approx 8 \%$ when both relaxed and unrelaxed clusters are included and evolution of its normalization with redshift is consistent with expectations of the self-similar model. The insensitivity of the relation the dynamical state of clusters is not trivial and is due to the fact that during mergers clusters move almost exactly along the relation (e.g., Poole et al. 2007, Rasia et al. 2011). In addition, the slope and normalization of the $Y_{\mathrm{X}}-M_{500}$ relation is also not sensitive to specific assumptions in modelling cooling and feedback heating processes in simulations (Fabjan et al. 2011, Stanek et al. 2010), which makes them more robust theoretically.

The $Y_{S Z}-M$ relation also exhibits a comparably low-scatter and the slope and evolution of normalization are close to the predictions of the self-similar model da Silva et al. 2004, Motl et al. 2005), which is not surprising given the similarity between the $Y_{\mathrm{X}}$ and the integrated $Y_{\mathrm{SZ}}$ measured from SZ observations. Its normalization changes by up to 30-40\% depending on the interplay between radiative cooling and feedback processes included in the simulations (e.g. Battaglia et al. 2011, Bonaldi et al. 2007, Nagai 2006, and references therein). At the same time, simulation analysis is also shedding light on the effect of projection (e.g. Kay et al. 2012) and mergers (e.g. Krause et al. 2012) on the scatter in the $Y_{S Z}-M$ scaling.

The tight relation of integral quantities such as $Y_{\mathrm{X}}, Y_{\mathrm{SZ}}, M_{\mathrm{g}}$, or core-excised X-ray luminosity with the total mass makes them good proxies for observational estimates of cluster mass, which can be used at high redshifts even with a relatively small number of X-ray photons. For instance, integral measurements of gas mass or temperature requires $\sim 10^{3}$ photons, which is feasible for statistically complete cluster samples out to z $\sim 1$ (e.g., Mantz et al. 2010a, Maughan 2007, Vikhlinin et al. 2009a) or even beyond. This makes these integral quantities very useful as "mass proxies" in cosmological analyses of cluster populations (e.g., Allen, Evrard \& Mantz 2011). Clearly, the relation of such mass proxies with the actual mass needs to be calibrated both via detailed observations of small controlled cluster samples and in cosmological simulations of cluster formation.

The potential danger of relying on simulations for this calibration is that results could be sensitive to the details of the physical processes included. This implies that a mass proxy is required to have not only a low scatter in its scaling with mass, but also to be robust against changing the uncertain description of the ICM physics. As we noted above, $Y_{\mathrm{X}}$ is quite robust to changes within a wide range of assumptions about cooling and heating processes affecting the ICM. This is illustrated in Figure 15 (taken from Fabjan et al. 2011) which shows how the normalization and slope of the scaling relation of gas mass and $Y_{X}$ versus $M_{500}$ change with the physical processes included. The evolution of the $Y_{\mathrm{X}}-M_{500}$ relation with redshift is also consistent with self-similar expectations for different models of cooling and feedback. Other quantities, such as $M_{\mathrm{g}}$, often exhibit a similar or even smaller 


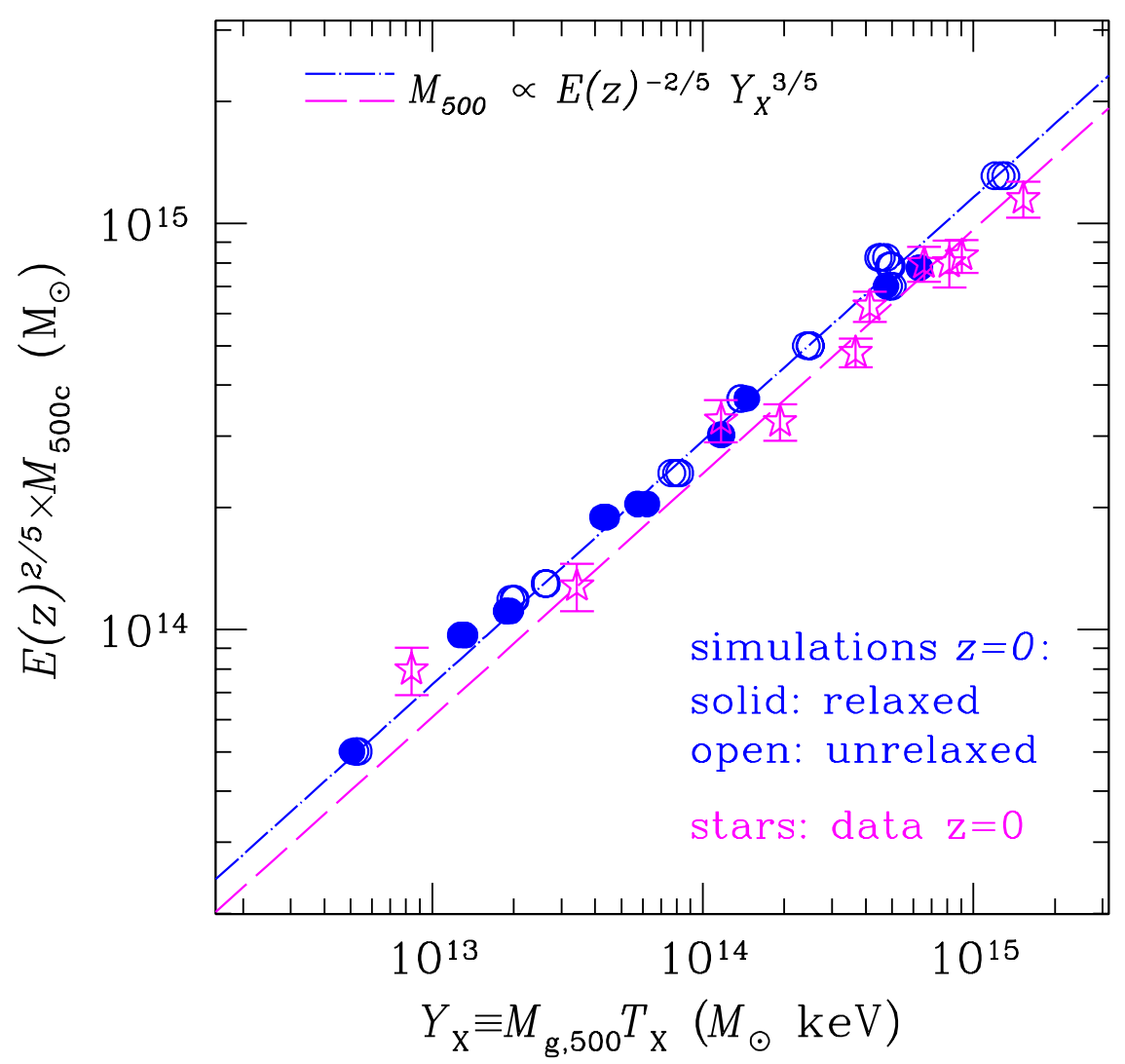

Fig. 14. The $Y_{X}-M_{500}$ relation for a set of simulated clusters at $\mathrm{z}=0$ (circles) and for a sample of relaxed Chandra clusters from Vikhlinin et al. (2006) (stars with errorbars). Filled and open circles refer to simulated clusters which are classified as relaxed and unrelaxed, respectively. Core regions inside $0.15 r_{500}$ are excised in the measurement of the X-ray temperature entering into the computation of $Y_{X}$, for both simulated and real clusters. True and hydrostatic masses are shown for simulated and observed clusters, respectively. The dot-dashed line shows the best-fit power-law relation for the simulated clusters with the slope fixed to the self-similar value of alpha $=3 / 5$. The dashed line shows the same best-fit power-law relation to simulations, but with the normalization scaled down by $15 \%$, which takes into account the putative effect of hydrostatic mass bias due to residual gas motions. From Kravtsov, Vikhlinin \& Nagai (2006).

degree of scatter compared to $Y_{\mathrm{X}}$ but are more sensitive to the choice of physical processes included in simulations. An additional practical consideration is that theoretical models should consider observables derived from mock observations of simulated clusters that take into account instrumental effects of detectors and projection effects (e.g., Biffi et al. 2011; Nagai, Vikhlinin \& Kravtsov 2007; ; Rasia et al. 2006).

Ultimately, calibration of mass proxies for precision use should be obtained via independent observational mass measurements, using the weak lensing analysis, HE, or velocity dispersions of member galaxies. The combination of future large, wide-area X-ray, SZ, and optical/near-IR surveys should provide a significant progress in this direction. 


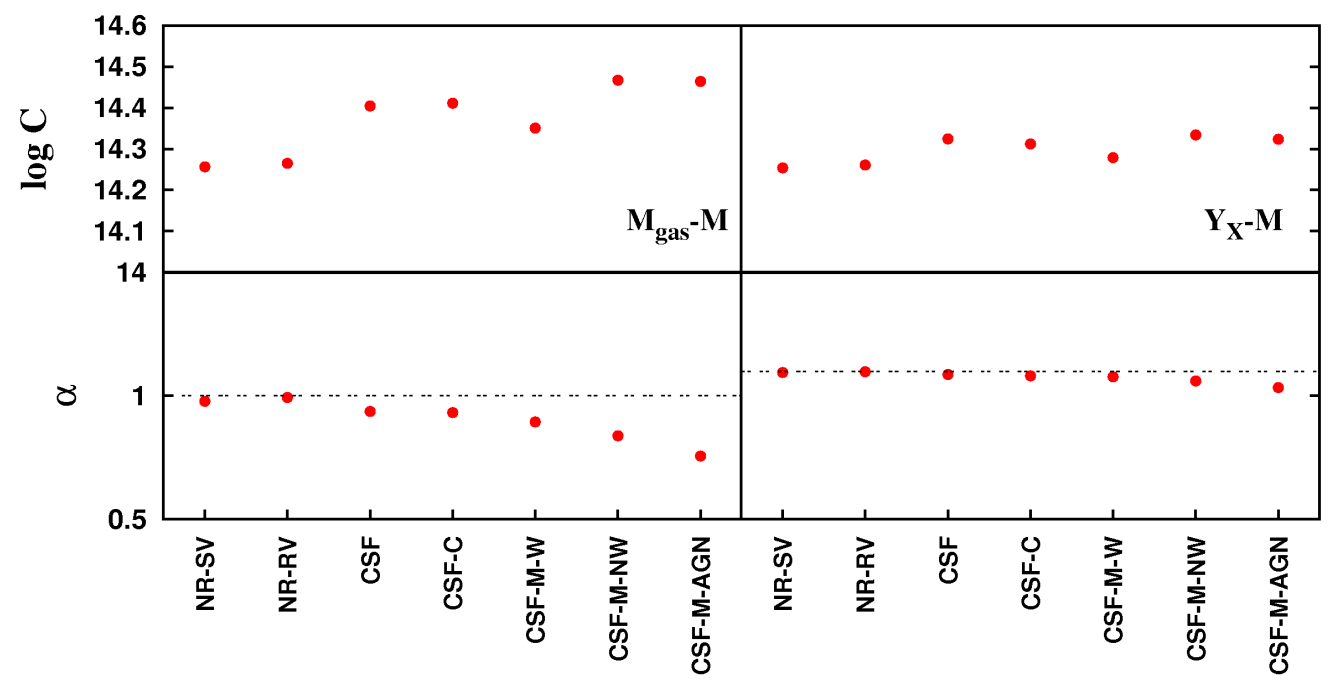

Fig. 15. Sensitivity of different mass proxies on the physical description of the intracluster medium included in cosmological hydrodynamical simulations for a set of galaxy clusters (from Fabjan et al. 2011). Results for the scaling relation of $M_{500}$ with gas mass $M_{\text {gas }}$ and $Y_{X}$ are shown in the left and right panels, respectively. Best fitting normalization $C$ and slope $\alpha$ of the scaling relations of these three mass proxies are shown in the upper and lower panels, respectively. Here, $T_{\mathrm{mw}}$ is the mass-weighted temperature computed excluding the central cluster regions within $0.15 r_{500}$. In the lower panels, the horizontal dashed lines mark the values of the slope of the scaling relations predicted by the self-similar model. Results are shown for simulations only including non-radiative hydrodynamics with standard (NR-SV) and reduced artical viscosity (NR-RV); cooling and star formation without (CSF) and with thermal conduction (CSF-C); cooling and star formation with metal enrichment, with (CSF-M-W) and without (CSF-M-NW) galactic winds from SN explosions; and cooling and star formations with the eect of AGN feedback (CSF-M-AGN) (see Fabjan et al. 2011 for further details).

\section{Cluster formation in alternative cosmological models}

In previous sections, we have discussed the main elements of cluster formation in the standard $\Lambda C D M$ cosmology. Although this model is very successful in explaining a wide variety of observations, some of its key assumptions and ingredients are not yet fully tested. This provides motivation to explore different assumptions and alternative models.

As discussed in Section 3.7, the halo mass function for a Gaussian random field is uniquely specified by the peak height $v=\delta_{c} / \sigma(R, \mathrm{z})$, where $R$ is the filtering scale corresponding to the cluster mass scale $M$. For sufficiently large mass, that is rare peaks with $v \gg 1$, the mass function becomes exponentially sensitive to the value of $v$. At the same time, the mass function also determines the halo bias (see Section 3.8). Again, for $v \gg 1$ and Gaussian perturbations, the bias function scales as $b(v) \sim v^{2} / \delta_{c}=v / \sigma(R, \mathrm{z})$. Therefore, the cluster 2-point correlation function can be written as $\xi_{\mathrm{cl}}(r)=v^{2}\left(\xi_{R}(r) / \sigma_{R}^{2}\right)$, where $\xi_{R}(r)$ is the correlation function of the smoothed fluctuation field (see Section 3.1). Once the peak 
height $v$ is constrained by requiring a model to predict the observed cluster abundance, the value of the cluster correlation function at a single scale $r$ provides a measurement of the shape of the power spectrum through the ratio of the clustering strength at the scale $r$ and at the cluster characteristic scale $R$. These predictions are only valid under two assumptions, namely Gaussianity of primordial density perturbations and scale independence of the linear growth function $D(\mathrm{z})$, as predicted by the standard theory of gravity. Therefore, the combination of number counts and large-scale clustering studies offers a powerful means to constrain the possible violation of either one of these two assumptions that hold for the $\Lambda \mathrm{CDM}$ model.

In this section, we briefly review the specifics of cluster formation in models with nonGaussian initial density field and with non-standard gravity, the most frequently discussed modifications to the standard structure formation paradigm.

\subsection{Mass function and bias of clusters in non-Gaussian models}

One of the key assumptions of the standard model of structure formation is that initial density perturbations are described by a Gaussian random field (see Section 3.1). The simplest single-field, slow-roll inflation models predict nearly Gaussian initial density fields. However, deviations from Gaussianity are expected in a broad range of inflation models that violate slow-roll approximation, and have multiple fields, or modified kinetic terms (see Bartolo et al. 2004, for a review). Given that there is no single preferred inflation model, we do not know which specific form of non-Gaussianity is possibly realized in nature. Deviations from Gaussianity are parameterized using a heuristic functional form. One of the simplest and most common choices for such a form is the local non-Gaussian potential given by $\Psi_{\mathrm{NG}}(\mathbf{x})=-\left(\phi_{\mathrm{G}}(\mathbf{x})+f_{\mathrm{NL}}\left[\phi_{\mathrm{G}}(\mathbf{x})^{2}-\left\langle\phi_{\mathrm{G}}^{2}\right\rangle\right]\right)$, where $\Psi_{\mathrm{NG}}$ is the usual Newtonian potential, $\phi_{\mathrm{G}}$ is the Gaussian random field with zero mean, and the parameter $f_{\mathrm{NL}}=$ const controls the degree and nature of non-Gaussianity (e.g., Komatsu \& Spergel 2001; Matarrese, Verde \& Jimenez 2000; Salopek \& Bond 1990). The simplest inflation models predict $f_{\mathrm{NL}} \approx 10^{-2}$ (e.g., Maldacena 2003), but a number of models that predict much larger degree of non-Gaussianity exist as well (Bartolo et al. 2004). The current CMB constraint on scaleindependent non-Gaussianity is $f_{\mathrm{NL}}=30 \pm 20$ at the $68 \%$ confidence level (e.g., Komatsu $2010)$ and there is thus still room for existence of sizable deviations from Gaussianity.

The non-Gaussian fields with $f_{\mathrm{NL}}<0$ have a PDF of the potential field that is skewed toward positive values and the abundance of peaks that seed the collapse of halos is reduced compared to Gaussian initial conditions. Conversely, the PDF of the potential field in models with $f_{\mathrm{NL}}>0$ has negative skewness, and hence an increased number of potential minima (density peaks). This would result in an enhanced abundance of rare objects, such as massive distant clusters, relative to the Gaussian case (see, e.g., figure 1 in Dalal et al. 2008 , for an illustration of the effect of $f_{\mathrm{NL}}$ on the large-scale structure that forms). The suppression or enhancement of abundance of halos increases with increasing peak height.

The mass functions resulting from non-Gaussian initial conditions have been studied both analytically (e.g., Afshordi \& Tolley 2008; Chiu, Ostriker \& Strauss 1998; Lo Verde et al. 2008; Matarrese, Verde \& Jimenez 2000) and using cosmological simulations (Dalal et al. 2008, Grossi et al. 2007, Lo Verde et al. 2008, LoVerde \& Smith 2011, Wagner \& Verde 2012). These studies showed that accurate formulae for the halo abundance from the initial linear density field exist for the non-Gaussian models as well. The general result is that the fractional change in the abundance of the rarest peaks is of order unity for the initial fields 
with $\left|f_{\mathrm{NL}}\right| \sim 100$. The abundance of clusters is thus only mildly sensitive to deviations of Gaussianity within the currently constrained limits (Cunha, Huterer \& Doré 2010, Sartoris et al. 2010; Scoccimarro, Sefusatti \& Zaldarriaga 2004; Sefusatti et al. 2007). In contrast, primordial non-Gaussianity may also leave an imprint in the spatial distribution of clusters in the form of a scale-dependence of large-scale linear bias.

As was discovered by Dalal et al. (2008) and confirmed in subsequent analytical (Afshordi \& Tolley 2008; Matarrese \& Verde 2008; McDonald 2008; Slosar et al. 2008; Taruya, Koyama \& Matsubara 2008) and numerical studies (Desjacques, Seljak \& Iliev 2009; Grossi et al. 2009, Pillepich, Porciani \& Hahn 2010; Shandera, Dalal \& Huterer 2011, the linear bias of collapsed objects in the models with local non-Gaussianity can be described as a function of wavenumber $k$ by $b_{\mathrm{NG}}=b_{\mathrm{G}}+f_{\mathrm{NL}} \times$ const $/ k^{2}$, where $b_{\mathrm{G}}$ is the linear bias in the corresponding cosmological model with the Gaussian initial conditions discussed in $\S 3.8$. This scale dependence arises because in the non-Gaussian models the large-scale modes that boost the abundance of peaks are correlated with the peaks themselves, which enhances (or suppresses) the peak amplitudes by a factor proportional to $f_{\mathrm{NL}} \phi \propto f_{\mathrm{NL}} \delta / k^{2}$. Because this effect of modulation increases with increasing peak height, $v=\delta_{c} / \sigma(M, \mathrm{z})$, the scale-dependence of bias increases with increasing halo mass. This unique signature can be used as a powerful constraint on deviations from Gaussianity (at least for models with local non-Gaussianity) in large samples of clusters in which the power spectrum or correlation function can be measured on large scales.

\subsection{Formation of clusters in modified gravity models}

Recently, there has been a renewed interest in modifications to the standard GR theory of gravity (e.g., see Capozziello \& de Laurentis 2011, Durrer \& Maartens 2008, Silvestri \& Trodden 2009, for recent reviews). These models have implications not only for cosmic expansion, but also for the evolution of density perturbations and, therefore, for the formation of galaxy clusters.

For instance, in the class of the $f(R)$ models, cosmic acceleration arises from a modification of gravity law given by the addition of a general function $f(R)$ of the Ricci curvature scalar $R$ in the Einstein-Hilbert action (see, e.g., Jain \& Khoury 2010, Sotiriou \& Faraoni 2010, for recent reviews). Such modifications result in enhancements of gravitational forces on scales relevant for structure formation in such a way that the resulting linear perturbation growth rate $D$ becomes scale dependent; whereas on very large scales gravity behaves similarly to GR gravity, on smaller scales it is enhanced compared to GR and the rate of structure formation is thereby also enhanced. The nonlinear halo collapse and growth are also faster in $f(R)$ models, which leads to enhanced abundance of massive clusters (Ferraro, Schmidt \& Hu 2011; Schmidt et al. 2009; Zhao, Li \& Koyama 2011) compared to the predictions of the models with GR gravity and identical cosmological parameters. Likewise, the peaks collapsing by a given $\mathrm{z}$ have lower peak height $v$ in the modified gravity models compared to the peak height in the standard gravity model. This results in the reduced bias of clusters of a given mass compared to the standard model. Furthermore, the scale dependence of the linear growth also induces a scale dependence of bias, thus offering another route to detect modifications of gravity (Parfrey, Hui \& Sheth 2011). Qualitatively similar effects on cluster abundance and bias are expected in the braneworld-modified gravity models based on higher dimensions, such as the Dvali-Gabadadze-Porrati (DGP, Dvali, Gabadadze \& Porrati 2000) gravity model (Khoury \& Wyman 2009; Schäfer \& Koyama 2008; Schmidt 2009; Schmidt, Hu \& Lima 2010) and its successors with similar LSS phenomenology 


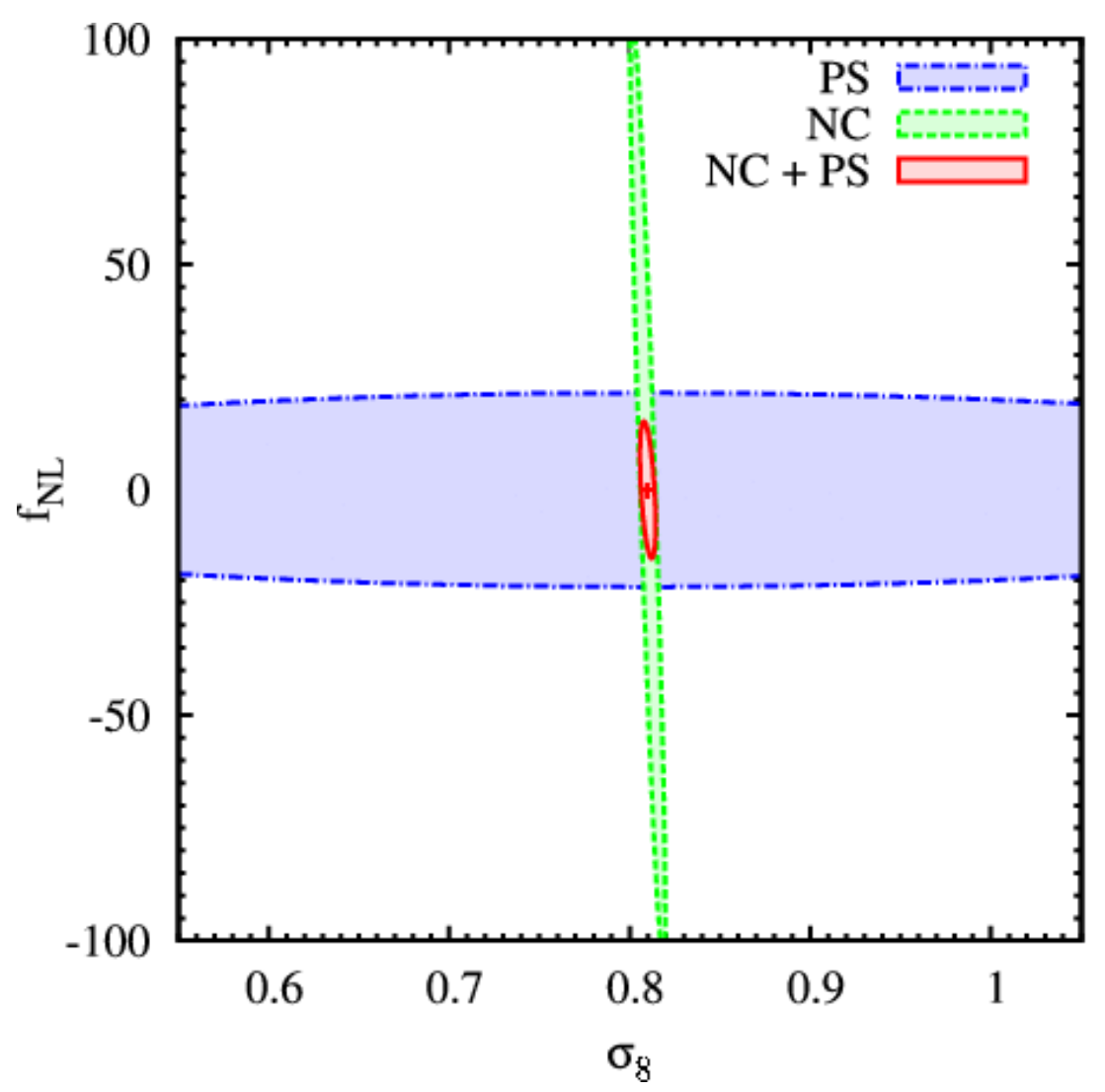

Fig. 16. The potential of future cluster X-ray surveys to constrain deviations from Gaussian density perturbations (adapted from Sartoris et al. 2010). The figure shows constraints on the power-spectrum normalization, $\sigma_{8}$, and non-Gaussianity parameter, $f_{\mathrm{NL}}$, expected from surveys of galaxy clusters to be carried out with the next-generation Wide Field X-ray Telescope. Dot-dashed blue curve and dashed green curve show the $68 \%$ confidence regions provided by the evolution of power spectrum (PS) of the cluster distribution and cluster number counts (NC), respectively. The solid red ellipse shows the constraints obtained by combining number counts and power spectrum information. Cocmic Microwave Background Planck priors for Gaussian perturbations have been included in the analysis.

consistent with current observational constraints, such as models of ghost-free massive gravity (D’Amico et al. 2011; de Rham, Gabadadze \& Tolley 2011).

A general consequence of modifying gravity is that the Birkhoff theorem no longer holds, which does not allow a straightforward extension of the spherical collapse model described in Section 3.2 to a generic model of modified gravity. Nevertheless, numerical calculations of spherical collapse have been presented for a number of specific models (e.g., Martino, Stabenau \& Sheth 2009; Schäfer \& Koyama 2008; Schmidt, Hu \& Lima 2010; Schmidt et al. 2009). For both the $f(R)$ and the DGP classes of models, the results of simulations obtained so far suggest that halo mass function and bias can still be described by the universal functions of peak height, in which the threshold for collapse and the linear growth rate are modified appropriately from their standard model values (Schmidt, Hu \& Lima 2010; Schmidt et al. 2009). This implies that it should be possible to calibrate mass function and bias of halos in the modified gravity models with the accuracy comparable to that in the standard structure formation models. 


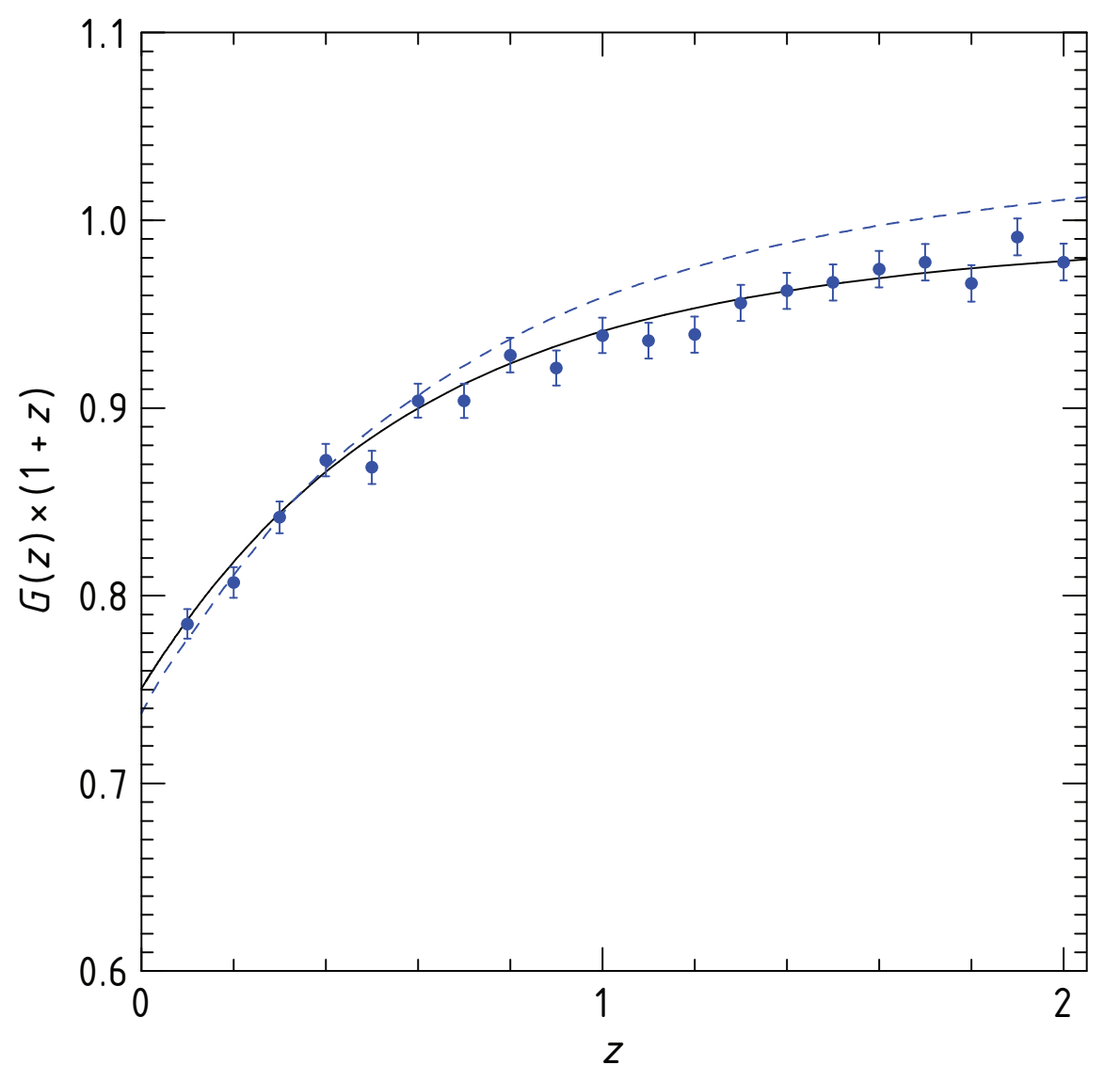

Fig. 17. The potential of future cluster surveys to constrain deviations from General Relativity (from Vikhlinin et al. 2009d). The linear growth factor of density perturbations, $G(\mathrm{z})=D(\mathrm{z})$ (not normalized to unity at $\mathrm{z}=0$ ), recovered from 2000 clusters, distributed in 20 redshift bins, each containing 100 massive clusters, identified in a high-sensitivity X-ray cluster survey. The solid black line indicates the evolution of the linear growth factor for a $\Lambda C D M$ model, whereas the dashed blue curve is the prediction of a modified gravity model (the brane world model by Dvali, Gabadadze \& Porrati 2000 ), having the same expansion history of the $\Lambda$ CDM model.

\section{Summary and outlook}

All of the main elements of the overall narrative of how clusters form and evolve discussed in this review have been established over the past four decades. The remarkable progress in our understanding of cluster formation has been accompanied by great progress in multiwavelength observations of clusters and our knowledge of the properties of the main mass constituents of clusters: stars, hot intracluster gas, and gravitationally dominant DM.

Formation of galaxy clusters is a complicated, non-linear process accompanied by a host of physical phenomena on a wide range of scales. Yet, some aspects of clusters exhibit remarkable regularity, and their internal structure, abundance, and spatial distribution carry an indelible memory of the initial linear density perturbation field and the cosmic expansion history. This is manifested both by tight scaling relations between cluster properties and the total mass, as well as by the approximate universality of the cluster mass function and bias, 
when expressed as a function of the peak height $v$.

Likewise, there is abundant observational evidence that complex processes - in the form of a non-linear, self-regulating cycle of gas cooling and accretion onto the SMBHs and associated feedback - have been operating in the central regions of clusters. In addition, the ICM is stirred by continuing accretion of the intergalactic gas, motion of cluster galaxies, and AGN bubbles. Studies of cluster cores provide a unique window into the interplay between the evolution of the most massive galaxies, taking place under extreme environmental conditions, and the physics of the diffuse hot baryons. At the same time, processes accompanying galaxy formation also leave a mark on the ICM properties at larger radii. In these regions, the gas entropy measured from observations is considerably higher than predicted by simple models that do not include such processes, and the ICM is also significantly enriched by heavy elements. This highlights that the ICM properties are the end product of the past interaction between the galaxy evolution processes and the intergalactic medium. Nevertheless, at intermediate radii, $r_{2500} \lesssim r \lesssim r_{500}$, the scaling of the radial profiles of gas density, temperature, and pressure with the total mass is close to simple, self-similar expectations for clusters of sufficiently large mass (corresponding to $k T \gtrsim 2-3 \mathrm{keV}$ ). This implies that the baryon processes affecting the ICM during cluster formation do not introduce a new mass scale. Such regular behaviour of the ICM profiles provides a basis for the definition of integrated quantities, such as the core-excised X-ray luminosity and temperature, gas mass, or integrated pressure, which are tightly correlated with each other and with the total cluster mass.

The low-scatter scaling relations are used to interpret abundance and spatial distribution of clusters and derive cosmological constraints (see Allen, Evrard \& Mantz 2011 and Weinberg et al. 2012 for recent reviews). Currently, cluster counts measured at high redshifts provide interesting constraints on cosmological parameters complementary to other methods (e.g., Mantz et al. 2010b, Rozo et al. 2010, Vikhlinin et al. 2009c) and a crucial test of the entire class of $\Lambda \mathrm{CDM}$ and quintessence models (e.g., Benson et al. 2011; Jee et al. 2011; Mortonson, Hu \& Huterer 2011). Although the statistical power of large future cluster surveys will put increasingly more stringent requirements on the theoretical uncertainties associated with cluster scaling relations and mass function (Cunha \& Evrard 2010; Wu, Zentner \& Wechsler 2010), future cluster samples can provide competitive constraints on the non-Gaussianity in the initial density field and deviations from GR gravity.

A combination of cluster abundance and large-scale clustering measurements can be used to derive stringent constraints on cosmological parameters and possible deviations from the standard $\Lambda \mathrm{CDM}$ paradigm. As an example, Figure 16 shows the constraints on the normalization of the power spectrum and the $f_{\mathrm{NL}}$ parameter, (from Sartoris et al. 2010) expected for a future high-sensitivity X-ray cluster survey. It shows that future cluster surveys can achieve a precision of $\sigma_{f_{\mathrm{NL}}} \approx 5-10$ (see also Cunha, Huterer \& Doré 2010; Pillepich, Porciani \& Reiprich 2012, thus complementing at smaller scales constraints on non-Gaussianity, which are to be provided on larger scales by observations of CMB anisotropies from the Planck satellite.

Although a variety of methods will provide constraints on the equation of state of DE and other cosmological parameters (e.g., Weinberg et al. 2012), clusters will remain one of the most powerful ways to probe deviations from the GR gravity (e.g., Lombriser et al. 2009). Even now, the strongest constraints on deviations from the GR on the Hubble horizon scales are derived from the combination of the measured redshift evolution of cluster number counts and geometrical probes of cosmic expansion (Schmidt, Vikhlinin \& Hu 2009). Fig- 
ure 17 illustrates the potential constraints on the linear rate of perturbation growth that can be derived from a future high-sensitivity X-ray cluster survey using similar analysis. The figure shows that a sample of about 2000 clusters at $\mathrm{z}<2$ with well-calibrated mass measurements would allow one to distinguish the standard $\Lambda \mathrm{CDM}$ model from a braneworldmodified gravity model with the identical expansion history at a high confidence level.

The construction of such large, homogeneous samples of clusters will be aided in the next decade by a number of cluster surveys both in the optical/near-IR (e.g., DES, PanSTARRS, EUCLID) and X-ray (e.g., eROSITA, WFXT) bands. At the same time, the combination of higher resolution numerical simulations including more sophisticated treatment of galaxy formation processes and high-sensitivity multi-wavelength observations of clusters should help to unveil the nature of the physical processes driving the evolution of clusters and provide accurate calibrations of their masses. The cluster studies thus will remain a vibrant and fascinating area of modern cosmology for years to come.

\section{Acknowledgments}

We are grateful to Brad Benson, Klaus Dolag, Surhud More, Piero Rosati, Elena Rasia, Ming Sun, Paolo Tozzi, Alexey Vikhlinin, Mark Voit, and Mark Wyman for useful discussions and comments, and to John Carlstrom for a careful reading of the manuscript. We thank Dunja Fabjan and Barbara Sartoris for their help in producing Fig. 15 and Fig. 16, respectively. The authors wish to thank the Kavli Institute for Theoretical Physics (KITP) in Santa Barbara for hospitality during the early phase of preparation of this review and participants of the KITP workshop "Galaxy clusters: crossroads of astrophysics and cosmology" for many stimulating discussions. AK was supported by NSF grants AST-0507596 and AST-0807444, NASA grant NAG5-13274, and by the Kavli Institute for Cosmological Physics at the University of Chicago through grants NSF PHY-0551142 and PHY-1125897. SB acknowledges partial support by the European Commissions FP7 Marie Curie Initial Training Network CosmoComp (PITN-GA-2009-238356), by the PRIN-INAF09 project "Towards an Italian Network for Computational Cosmology", by the PRIN-MIUR09 "Tracing the growth of structures in the Universe" and by the PD51 INFN grant. 


\section{References}

[1] Abadi MG, Navarro JF, Fardal M, Babul A, Steinmetz M. 2010. MNRAS 407:435-446

[2] Abramo LR, Batista RC, Liberato L, Rosenfeld R. 2007. JCAP 11:12

[3] Ackermann M, Ajello M, Allafort A, Baldini L, Ballet J, et al. 2010. ApJ 717:L71-L78

[4] Afshordi N, Tolley AJ. 2008. Phys. Rev. D 78:123507

[5] Alimi JM, Füzfa A, Boucher V, Rasera Y, Courtin J, Corasaniti PS. 2010. MNRAS 401:775790

[6] Allen SW, Evrard AE, Mantz AB. 2011. ARAA 49:409-470

[7] Allen SW, Rapetti DA, Schmidt RW, Ebeling H, Morris RG, Fabian AC. 2008. MNRAS 383:879-896

[8] Allen SW, Schmidt RW, Ebeling H, Fabian AC, van Speybroeck L. 2004. MNRAS 353:457467

[9] Allgood B, Flores RA, Primack JR, Kravtsov AV, Wechsler RH, et al. 2006. MNRAS 367:1781-1796

[10] Ameglio S, Borgani S, Pierpaoli E, Dolag K, Ettori S, Morandi A. 2009. MNRAS 394:479_ 490

[11] Anderhalden D, Diemand J. 2011. MNRAS 414:3166-3172

[12] Arnaud M, Pratt GW, Piffaretti R, Böhringer H, Croston JH, Pointecouteau E. 2010. AE A 517:A92

[13] Ascasibar Y, Sevilla R, Yepes G, Müller V, Gottlöber S. 2006. MNRAS 371:193-203

[14] Audit E, Teyssier R, Alimi JM. 1997. AEFA 325:439-449

[15] Babul A, Balogh ML, Lewis GF, Poole GB. 2002. MNRAS 330:329-343

[16] Bahcall NA, Soneira RM. 1983. ApJ 270:20-38

[17] Bardeen JM, Bond JR, Kaiser N, Szalay AS. 1986. ApJ 304:15-61

[18] Bardeen JM, Steinhardt PJ, Turner MS. 1983. Phys. Rev. D 28:679-693

[19] Barnes J, White SDM. 1984. MNRAS 211:753-765

[20] Bartelmann M. 2010. Classical and Quantum Gravity 27:233001

[21] Bartolo N, Komatsu E, Matarrese S, Riotto A. 2004. Phys. Rep. 402:103-266

[22] Battaglia N, Bond JR, Pfrommer C, Sievers JL. 2011. ApJ in press (arXiv/1109.3709)

[23] Becker MR, Kravtsov AV. 2011. ApJ 740:25

[24] Benson BA, de Haan T, Dudley JP, Reichardt CL, Aird KA, et al. 2011. ApJ in press $(\operatorname{arXiv} / 1112.5435)$

[25] Bertschinger E. 1985. ApJS 58:39-65

[26] Bertschinger E. 1998. ARAA 36:599-654

[27] Bhattacharya S, Habib S, Heitmann K. 2011. ApJ in press (arXiv/1112.5479)

[28] Bhattacharya S, Heitmann K, White M, Lukić Z, Wagner C, Habib S. 2011. ApJ 732:122

[29] Bialek JJ, Evrard AE, Mohr JJ. 2001. ApJ 555:597-612

[30] Biffi V, Dolag K, Boehringer H, Lemson G. 2011. MNRAS in press (arXiv/1112.0314)

[31] Biffi V, Dolag K, Böhringer H. 2011. MNRAS 413:573-584

[32] Binney J, Tremaine S. 2008. Galactic Dynamics: Second Edition. Princeton University Press

[33] Blumenthal GR, Faber SM, Flores R, Primack JR. 1986. ApJ 301:27-34

[34] Blumenthal GR, Faber SM, Primack JR, Rees MJ. 1984. Nature 311:517-525

[35] Böhringer H, Belsole E, Kennea J, Matsushita K, Molendi S, et al. 2001. AEFA 365:L181L187

[36] Böhringer H, Dolag K, Chon G. 2012. AE $A$ 539:A120

[37] Böhringer H, Schuecker P, Pratt GW, Arnaud M, Ponman TJ, et al. 2007. AEFA 469:363377

[38] Bonaldi A, Tormen G, Dolag K, Moscardini L. 2007. MNRAS 378:1248-1258 
[39] Bonamente M, Hasler N, Bulbul E, Carlstrom JE, Culverhouse TL, et al. 2012. New Journal of Physics 14:025010

[40] Bond JR, Efstathiou G. 1984. ApJ 285:L45-L48

[41] Bond JR, Kofman L, Pogosyan D. 1996. Nature 380:603-605

[42] Bond JR, Szalay AS, Turner MS. 1982. Physical Review Letters 48:1636-1639

[43] Borgani S, Fabjan D, Tornatore L, Schindler S, Dolag K, Diaferio A. 2008. Space Science Reviews 134:379-403

[44] Borgani S, Finoguenov A, Kay ST, Ponman TJ, Springel V, et al. 2005. MNRAS 361:233243

[45] Borgani S, Governato F, Wadsley J, Menci N, Tozzi P, et al. 2002. MNRAS 336:409-424

[46] Borgani S, Kravtsov A. 2011. Adv. Sci. Letters 4:204-227

[47] Borgani S, Murante G, Springel V, Diaferio A, Dolag K, et al. 2004. MNRAS 348:10781096

[48] Borgani S, Viel M. 2009. MNRAS 392:L26-L30

[49] Bower RG, McCarthy IG, Benson AJ. 2008. MNRAS 390:1399-1410

[50] Brunetti G. 2011. Mem. SAIt 82:515-522

[51] Bryan GL, Norman ML. 1998. ApJ 495:80-99

[52] Bullock JS, Kolatt TS, Sigad Y, Somerville RS, Kravtsov AV, et al. 2001. MNRAS 321:559_ 575

[53] Buote DA, Gastaldello F, Humphrey PJ, Zappacosta L, Bullock JS, et al. 2007. ApJ 664:123-134

[54] Capozziello S, de Laurentis M. 2011. Phys. Rep. 509:167-321

[55] Cardone VF, Piedipalumbo E, Tortora C. 2005. MNRAS 358:1325-1336

[56] Carlstrom JE, Holder GP, Reese ED. 2002. ARAA 40:643-680

[57] Cavagnolo KW, Donahue M, Voit GM, Sun M. 2009. ApJS 182:12-32

[58] Cavaliere AG, Gursky H, Tucker WH. 1971. Nature 231:437-438

[59] Chiu WA, Ostriker JP, Strauss MA. 1998. ApJ 494:479-490

[60] Churazov E, Vikhlinin A, Zhuravleva I, Schekochihin A, Parrish I, et al. 2012. MNRAS 421:1123-1135

[61] Clowe D, De Lucia G, King L. 2004. MNRAS 350:1038-1048

[62] Cohn JD, White M. 2008. MNRAS 385:2025-2033

[63] Cole S, Kaiser N. 1989. MNRAS 237:1127-1146

[64] Cole S, Lacey C. 1996. MNRAS 281:716-736

[65] Colín P, Klypin AA, Kravtsov AV. 2000. ApJ 539:561-569

[66] Comerford JM, Natarajan P. 2007. MNRAS 379:190-200

[67] Conroy C, Ostriker JP. 2008. ApJ 681:151-166

[68] Cooray A, Sheth R. 2002. Phys. Rep. 372:1-129

[69] Corasaniti PS, Achitouv I. 2011. Physical Review Letters 106:241302

[70] Corless VL, King LJ. 2007. MNRAS 380:149-161

[71] Courtin J, Rasera Y, Alimi JM, Corasaniti PS, Boucher V, Füzfa A. 2011. MNRAS 410:1911-1931

[72] Creminelli P, D’Amico G, Noreña J, Senatore L, Vernizzi F. 2010. JCAP 3:27

[73] Crocce M, Fosalba P, Castander FJ, Gaztañaga E. 2010. MNRAS 403:1353-1367

[74] Croston JH, Pratt GW, Böhringer H, Arnaud M, Pointecouteau E, et al. 2008. AEAA 487:431-443

[75] Cuesta AJ, Prada F, Klypin A, Moles M. 2008. MNRAS 389:385-397

[76] Cui W, Borgani S, Dolag K, Murante G, Tornatore L. 2011. MNRAS in press (arXiv/1111.3066)

[77] Cunha C, Huterer D, Doré O. 2010. Phys. Rev. D 82:023004

[78] Cunha CE, Evrard AE. 2010. Phys. Rev. D 81:083509 
[79] da Silva AC, Kay ST, Liddle AR, Thomas PA. 2004. MNRAS 348:1401-1408

[80] Dalal N, Doré O, Huterer D, Shirokov A. 2008. Phys. Rev. D 77:123514

[81] Dalal N, Keeton CR. 2003. arxiv/0312072

[82] D’Amico G, de Rham C, Dubovsky S, Gabadadze G, Pirtskhalava D, Tolley AJ. 2011. Phys. Rev. D 84:124046

[83] Davis M, Efstathiou G, Frenk CS, White SDM. 1985. ApJ 292:371-394

[84] de Rham C, Gabadadze G, Tolley AJ. 2011. Physical Review Letters 106:231101

[85] de Simone A, Maggiore M, Riotto A. 2011. MNRAS 412:2587-2602

[86] Debattista VP, Moore B, Quinn T, Kazantzidis S, Maas R, et al. 2008. ApJ 681:1076-1088

[87] Desjacques V. 2008. MNRAS 388:638-658

[88] Desjacques V, Seljak U, Iliev IT. 2009. MNRAS 396:85-96

[89] Diemand J, Kuhlen M, Madau P. 2007. ApJ 667:859-877

[90] Diemand J, Moore B, Stadel J. 2005. Nature 433:389-391

[91] Dolag K, Borgani S, Schindler S, Diaferio A, Bykov AM. 2008. Sp. Sc. Rev. 134:229-268

[92] Dolag K, Bykov AM, Diaferio A. 2008. Space Science Reviews 134:311-335

[93] Dolag K, Jubelgas M, Springel V, Borgani S, Rasia E. 2004. ApJ 606:L97-L100

[94] Doroshkevich AG. 1970. Astrofizika 6:581-600

[95] Dubinski J. 1994. ApJ 431:617-624

[96] Dubinski J, Carlberg RG. 1991. ApJ 378:496-503

[97] Dubois Y, Devriendt J, Teyssier R, Slyz A. 2011. MNRAS 417:1853-1870

[98] Duffy AR, Schaye J, Kay ST, Dalla Vecchia C. 2008. MNRAS 390:L64-L68

[99] Durrer R, Maartens R. 2008. General Relativity and Gravitation 40:301-328

[100] Dvali G, Gabadadze G, Porrati M. 2000. Physics Letters B 485:208-214

[101] Eckert D, Molendi S, Gastaldello F, Rossetti M. 2011. AEFA 529:A133

[102] Efstathiou G, Davis M, White SDM, Frenk CS. 1985. ApJS 57:241-260

[103] Einasto J. 1965. Trudy Inst. Astrofiz. Alma-Ata 51:87

[104] Einasto J, Klypin AA, Saar E, Shandarin SF. 1984. MNRAS 206:529-558

[105] Eisenstein DJ, Hu W. 1999. ApJ 511:5-15

[106] Eke VR, Navarro JF, Frenk CS. 1998. ApJ 503:569-92

[107] El-Zant AA, Kim WT, Kamionkowski M. 2004. MNRAS 354:169-175

[108] Ettori S, Gastaldello F, Leccardi A, Molendi S, Rossetti M, et al. 2010. AEFA 524:A68

[109] Ettori S, Molendi S. 2011. Memorie della Societa Astronomica Italiana Supplementi 17:47-59

[110] Ettori S, Morandi A, Tozzi P, Balestra I, Borgani S, et al. 2009. AEFA 501:61-73

[111] Ettori S, Tozzi P, Rosati P. 2003. AEFA 398:879-890

[112] Evrard AE. 1988. MNRAS 235:911-934

[113] Evrard AE. 1990. ApJ 363:349-366

[114] Evrard AE, Henry JP. 1991. ApJ 383:95-103

[115] Evrard AE, MacFarland TJ, Couchman HMP, Colberg JM, Yoshida N, et al. 2002. ApJ 573:7-36

[116] Evrard AE, Summers FJ, Davis M. 1994. ApJ 422:11-36

[117] Fabian AC. 1994. ARAA 32:277-318

[118] Fabjan D, Borgani S, Rasia E, Bonafede A, Dolag K, et al. 2011. MNRAS 416:801-816

[119] Fabjan D, Borgani S, Tornatore L, Saro A, Murante G, Dolag K. 2010. MNRAS 401:16701690

[120] Fabricant D, Gorenstein P. 1983. ApJ 267:535-546

[121] Fang T, Humphrey P, Buote D. 2009. ApJ 691:1648-1659

[122] Fedeli C. 2012. MNRAS in press (arXiv/1111.5780)

[123] Feldmann R, Carollo CM, Mayer L, Renzini A, Lake G, et al. 2010. ApJ 709:218-240

[124] Ferrari C, Govoni F, Schindler S, Bykov AM, Rephaeli Y. 2008. Space Science Reviews 
134:93-118

[125] Ferraro S, Schmidt F, Hu W. 2011. Phys. Rev. D 83:063503

[126] Fillmore JA, Goldreich P. 1984. ApJ 281:1-8

[127] Foley RJ, Andersson K, Bazin G, de Haan T, Ruel J, et al. 2011. ApJ 731:86

[128] Forman W, Kellogg E, Gursky H, Tananbaum H, Giacconi R. 1972. ApJ 178:309-316

[129] Frenk CS, White SDM, Davis M, Efstathiou G. 1988. ApJ 327:507-525

[130] Gao L, Navarro JF, Cole S, Frenk CS, White SDM, et al. 2008. MNRAS 387:536-544

[131] Gaspari M, Melioli C, Brighenti F, D'Ercole A. 2011. MNRAS 411:349-372

[132] Giovannini G, Bonafede A, Feretti L, Govoni F, Murgia M, et al. 2009. AE A 507:12571270

[133] Gnedin OY, Ceverino D, Gnedin NY, Klypin AA, Kravtsov AV, et al. 2011. ApJ in press (arXiv/1108.57360)

[134] Gnedin OY, Kravtsov AV, Klypin AA, Nagai D. 2004. ApJ 616:16-26

[135] Gott III JR, Gunn JE. 1971. ApJ 169:L13-15

[136] Gott III JR, Rees MJ. 1975. AEA 45:365-376

[137] Govoni F, Dolag K, Murgia M, Feretti L, Schindler S, et al. 2010. AEFA 522:A105

[138] Graham AW, Merritt D, Moore B, Diemand J, Terzić B. 2006. AJ 132:2701-2710

[139] Gross MAK, Somerville RS, Primack JR, Holtzman J, Klypin A. 1998. MNRAS 301:8194

[140] Grossi M, Dolag K, Branchini E, Matarrese S, Moscardini L. 2007. MNRAS 382:12611267

[141] Grossi M, Verde L, Carbone C, Dolag K, Branchini E, et al. 2009. MNRAS 398:321-332

[142] Gunn JE, Gott III JR. 1972. ApJ 176:1-19

[143] Guo F, Oh SP. 2008. MNRAS 384:251-266

[144] Gursky H, Kellogg E, Murray S, Leong C, Tananbaum H, Giacconi R. 1971. ApJ 167:L81-84

[145] Gustafsson M, Fairbairn M, Sommer-Larsen J. 2006. Phys. Rev. D 74:123522

[146] Guth AH, Pi SY. 1982. Physical Review Letters 49:1110-1113

[147] Hansen SH, Moore B. 2006. New Astronomy 11:333-338

[148] Heath DJ. 1977. MNRAS 179:351-358

[149] Hennawi JF, Dalal N, Bode P, Ostriker JP. 2007. ApJ 654:714-730

[150] Henning JW, Gantner B, Burns JO, Hallman EJ. 2009. ApJ 697:1597-1620

[151] Hoekstra H. 2007. MNRAS 379:317-330

[152] Hoffman Y, Shaham J. 1985. ApJ 297:16-22

[153] Hu W, Kravtsov AV. 2003. ApJ 584:702-715

[154] Hubble E, Humason ML. 1931. ApJ 74:43-80

[155] Hubble EP. 1925. The Observatory 48:139-142

[156] Hubble EP. 1926. ApJ 64:321-369

[157] Huchra JP, Geller MJ. 1982. ApJ 257:423-437

[158] Huss A, Jain B, Steinmetz M. 1999a. ApJ 517:64-69

[159] Huss A, Jain B, Steinmetz M. 1999b. MNRAS 308:1011-1031

[160] Inogamov NA, Sunyaev RA. 2003. Astronomy Letters 29:791-824

[161] Jain B, Khoury J. 2010. Annals of Physics 325:1479-1516

[162] Jee MJ, Dawson KS, Hoekstra H, Perlmutter S, Rosati P, et al. 2011. ApJ 737:59

[163] Jenkins A, Frenk CS, White SDM, Colberg JM, Cole S, et al. 2001. MNRAS 321:372-384

[164] Jing YP, Suto Y. 2002. ApJ 574:538-553

[165] Jing YP, Zhang P, Lin WP, Gao L, Springel V. 2006. ApJ 640:L119-L122

[166] Juncher D, Hansen SH, Macciò AV. 2012. ApJ 746:L28

[167] Kaiser N. 1984. ApJ 284:L9-L12

[168] Kaiser N. 1986. MNRAS 222:323-345 
REFERENCES

[169] Kaiser N. 1991. ApJ 383:104-111

[170] Kasun SF, Evrard AE. 2005. ApJ 629:781-790

[171] Katz N. 1991. ApJ 368:325-336

[172] Kay ST, da Silva AC, Aghanim N, Blanchard A, Liddle AR, et al. 2007. MNRAS 377:317334

[173] Kay ST, Peel MW, Short CJ, Thomas PA, Young OE, et al. 2012. MNRAS in press $(\operatorname{arXiv} / 1112.3769)$

[174] Kazantzidis S, Kravtsov AV, Zentner AR, Allgood B, Nagai D, Moore B. 2004. ApJ 611:L73-L76

[175] Kazantzidis S, Zentner AR, Kravtsov AV. 2006. ApJ 641:647-664

[176] Kellogg E, Gursky H, Tananbaum H, Giacconi R, Pounds K. 1972. ApJ 174:L65-69

[177] Khoury J, Wyman M. 2009. Phys. Rev. D 80:064023

[178] Klypin A, Borgani S, Holtzman J, Primack J. 1995. ApJ 444:1-14

[179] Klypin AA, Kopylov AI. 1983. Soviet Astronomy Letters 9:41-44

[180] Klypin AA, Shandarin SF. 1983. MNRAS 204:891-907

[181] Klypin AA, Trujillo-Gomez S, Primack J. 2011. ApJ 740:102

[182] Komatsu E. 2010. Classical and Quantum Gravity 27:124010

[183] Komatsu E, Smith KM, Dunkley J, Bennett CL, Gold B, et al. 2011. ApJS 192:18

[184] Komatsu E, Spergel DN. 2001. Phys. Rev. D 63:063002

[185] Krause E, Pierpaoli E, Dolag K, Borgani S. 2012. MNRAS 419:1766-1779

[186] Kravtsov AV, Vikhlinin A, Nagai D. 2006. ApJ 650:128-136

[187] Kravtsov AV, Yepes G. 2000. MNRAS 318:227-238

[188] Laganá TF, de Souza RS, Keller GR. 2010. AEFA 510:A76

[189] Lahav O, Lilje PB, Primack JR, Rees MJ. 1991. MNRAS 251:128-136

[190] Lapi A, Cavaliere A, Menci N. 2005. ApJ 619:60-72

[191] LaRoque SJ, Bonamente M, Carlstrom JE, Joy MK, Nagai D, et al. 2006. ApJ 652:917936

[192] Lau ET, Kravtsov AV, Nagai D. 2009. ApJ 705:1129-1138

[193] Lau ET, Nagai D, Kravtsov AV, Vikhlinin A, Zentner AR. 2012. ApJ in press (arxiv/1201.2168)

[194] Lau ET, Nagai D, Kravtsov AV, Zentner AR. 2011. ApJ 734:93

[195] Lawrence E, Heitmann K, White M, Higdon D, Wagner C, et al. 2010. ApJ 713:13221331

[196] Leccardi A, Molendi S. 2008. AEA 486:359-373

[197] Lee J, Evrard AE. 2007. ApJ 657:30-36

[198] Lee J, Shandarin SF. 1998. ApJ 500:14-27

[199] Lee J, Shandarin SF. 1999. ApJ 517:L5-L8

[200] Lee J, Springel V, Pen UL, Lemson G. 2008. MNRAS 389:1266-1274

[201] Lemze D, Wagner R, Rephaeli Y, Sadeh S, Norman ML, et al. 2011. ApJ in press (arxiv/1106.6048)

[202] Limber DN. 1959. ApJ 130:414-428

[203] Lin YT, Stanford SA, Eisenhardt PRM, Vikhlinin A, Maughan BJ, Kravtsov A. 2012. ApJ 745:L3

[204] Lithwick Y, Dalal N. 2011. ApJ 734:100

[205] Lo Verde M, Miller A, Shandera S, Verde L. 2008. JCAP 4:14

[206] Loken C, Norman ML, Nelson E, Burns J, Bryan GL, Motl P. 2002. ApJ 579:571-576

[207] Lombriser L, Hu W, Fang W, Seljak U. 2009. Phys. Rev. D 80:063536

[208] LoVerde M, Smith KM. 2011. JCAP 8:3

[209] Lukić Z, Heitmann K, Habib S, Bashinsky S, Ricker PM. 2007. ApJ 671:1160-1181

[210] Ma CP, Maggiore M, Riotto A, Zhang J. 2011. MNRAS 411:2644-2652 
REFERENCES

[211] Maggiore M, Riotto A. 2010. ApJ 717:515-525

[212] Maldacena J. 2003. Journal of High Energy Physics 5:13-55

[213] Mamon GA, Łokas EL. 2005. MNRAS 362:95-109

[214] Mandelbaum R, Seljak U, Hirata CM. 2008. JCAP 8:6-33

[215] Mantz A, Allen SW, Ebeling H, Rapetti D, Drlica-Wagner A. 2010a. MNRAS 406:17731795

[216] Mantz A, Allen SW, Rapetti D, Ebeling H. 2010b. MNRAS 406:1759-1772

[217] Marconi A, Hunt LK. 2003. ApJ 589:L21-L24

[218] Martino MC, Stabenau HF, Sheth RK. 2009. Phys. Rev. D 79:084013

[219] Martizzi D, Teyssier R, Moore B. 2012. MNRAS 420:2859-2873

[220] Matarrese S, Verde L. 2008. ApJ 677:L77-L80

[221] Matarrese S, Verde L, Jimenez R. 2000. ApJ 541:10-24

[222] Maughan BJ. 2007. ApJ 668:772-780

[223] Mazzotta P, Rasia E, Moscardini L, Tormen G. 2004. MNRAS 354:10-24

[224] McCarthy IG, Schaye J, Ponman TJ, Bower RG, Booth CM, et al. 2010. MNRAS 406:822839

[225] McDonald M, Veilleux S, Rupke DSN, Mushotzky R, Reynolds C. 2011. ApJ 734:95-106

[226] McDonald P. 2008. Phys. Rev. D 78:123519

[227] McNamara BR, Nulsen PEJ. 2007. ARAA 45:117-175

[228] Mead JMG, King LJ, Sijacki D, Leonard A, Puchwein E, McCarthy IG. 2010. MNRAS 406:434-444

[229] Meekins JF, Fritz G, Chubb TA, Friedman H. 1971. Nature 231:107-108

[230] Merritt D, Graham AW, Moore B, Diemand J, Terzić B. 2006. AJ 132:2685-2700

[231] Mo H, van den Bosch FC, White S. 2010. Galaxy Formation and Evolution (Cambridge University Press)

[232] Mo HJ, White SDM. 1996. MNRAS 282:347-361

[233] Monaco P. 1995. ApJ 447:23-34

[234] Moore B, Quinn T, Governato F, Stadel J, Lake G. 1999. MNRAS 310:1147-1152

[235] More S, Kravtsov AV, Dalal N, Gottlöber S. 2011. ApJS 195:4

[236] Mortonson MJ, Hu W, Huterer D. 2011. Phys. Rev. D 83:023015

[237] Moster BP, Somerville RS, Maulbetsch C, van den Bosch FC, Macciò AV, et al. 2010. ApJ 710:903-923

[238] Motl PM, Hallman EJ, Burns JO, Norman ML. 2005. ApJ 623:L63-L66

[239] Muanwong O, Thomas PA, Kay ST, Pearce FR. 2002. MNRAS 336:527-540

[240] Naab T, Johansson PH, Ostriker JP. 2009. ApJ 699:L178-L182

[241] Nagai D. 2006. ApJ 650:538-549

[242] Nagai D, Kravtsov AV, Vikhlinin A. 2007. ApJ 668:1-14

[243] Nagai D, Lau ET. 2011. ApJ 731:L10-L15

[244] Nagai D, Vikhlinin A, Kravtsov AV. 2007. ApJ 655:98-108

[245] Narayan R, Medvedev MV. 2001. ApJ 562:L129-L132

[246] Navarro JF, Frenk CS, White SDM. 1995. MNRAS 275:720-740

[247] Navarro JF, Frenk CS, White SDM. 1996. ApJ 462:563-575

[248] Navarro JF, Frenk CS, White SDM. 1997. ApJ 490:493-508

[249] Navarro JF, Hayashi E, Power C, Jenkins AR, Frenk CS, et al. 2004. MNRAS 349:1039_ 1051

[250] Nelson K, Rudd DH, Shaw L, Nagai D. 2011. ApJ in press (arXiv/1112.3659)

[251] Neumann DM. 2005. AEA 439:465-477

[252] Newman AB, Treu T, Ellis RS, Sand DJ. 2011. ApJ 728:L39-L43

[253] Newman AB, Treu T, Ellis RS, Sand DJ, Richard J, et al. 2009. ApJ 706:1078-1094

[254] Norman ML. 2010. arXiv/1005.1100 
[255] Oguri M, Bayliss MB, Dahle H, Sharon K, Gladders MD, et al. 2012. MNRAS 420:32133239

[256] Oguri M, Hennawi JF, Gladders MD, Dahle H, Natarajan P, et al. 2009. ApJ 699:10381052

[257] Oguri M, Takada M, Umetsu K, Broadhurst T. 2005. ApJ 632:841-846

[258] Okabe N, Zhang YY, Finoguenov A, Takada M, Smith GP, et al. 2010. ApJ 721:875-885

[259] Omma H, Binney J, Bryan G, Slyz A. 2004. MNRAS 348:1105-1119

[260] Onuora LI, Thomas PA. 2000. MNRAS 319:614-618

[261] O'Shea BW, Bryan G, Bordner J, Norman ML, Abel T, et al. 2004. arXiv/0403044

[262] Parfrey K, Hui L, Sheth RK. 2011. Phys. Rev. D 83:063511

[263] Peebles PJE. 1970. AJ 75:13-20

[264] Peebles PJE. 1982. ApJ 263:L1-L5

[265] Percival WJ. 2005. AEFA 443:819-830

[266] Peterson JR, Fabian AC. 2006. Phys.Reports 427:1-39

[267] Peterson JR, Paerels FBS, Kaastra JS, Arnaud M, Reiprich TH, et al. 2001. AEAA 365:L104-109

[268] Pfrommer C, Enßlin TA, Springel V, Jubelgas M, Dolag K. 2007. MNRAS 378:385-408

[269] Pfrommer C, Springel V, Enßlin TA, Jubelgas M. 2006. MNRAS 367:113-131

[270] Piffaretti R, Valdarnini R. 2008. AEFA 491:71-87

[271] Pillepich A, Porciani C, Hahn O. 2010. MNRAS 402:191-206

[272] Pillepich A, Porciani C, Reiprich TH. 2012. MNRAS 422:44-69

[273] Pointecouteau E, Arnaud M, Pratt GW. 2005. AEA 435:1-7

[274] Poole GB, Babul A, McCarthy IG, Fardal MA, Bildfell CJ, et al. 2007. MNRAS 380:437454

[275] Prada F, Klypin AA, Cuesta AJ, Betancort-Rijo JE, Primack J. 2012. MNRAS in press (arxiv/1104.5130)

[276] Prada F, Klypin AA, Simonneau E, Betancort-Rijo J, Patiri S, et al. 2006. ApJ 645:10011011

[277] Pratt GW, Arnaud M, Piffaretti R, Böhringer H, Ponman TJ, et al. 2010. AEGA 511:A85

[278] Pratt GW, Böhringer H, Croston JH, Arnaud M, Borgani S, et al. 2007. AEA 461:71-80

[279] Pratt GW, Croston JH, Arnaud M, Böhringer H. 2009. AE A 498:361-378

[280] Press WH, Davis M. 1982. ApJ 259:449-473

[281] Press WH, Schechter P. 1974. ApJ 187:425-438

[282] Puchwein E, Bartelmann M, Dolag K, Meneghetti M. 2005. AE A 442:405-412

[283] Puchwein E, Sijacki D, Springel V. 2008. ApJ 687:L53-L56

[284] Rasia E, Ettori S, Moscardini L, Mazzotta P, Borgani S, et al. 2006. MNRAS 369:20132024

[285] Rasia E, Mazzotta P, Evrard A, Markevitch M, Dolag K, Meneghetti M. 2011. ApJ 729:45

[286] Rasia E, Tormen G, Moscardini L. 2004. MNRAS 351:237-252

[287] Reed DS, Bower R, Frenk CS, Jenkins A, Theuns T. 2007. MNRAS 374:2-15

[288] Renzini A. 2000. In Large Scale Structure in the X-ray Universe, ed. M. Plionis \& I. Georgantopoulos

[289] Rosati P, Borgani S, Norman C. 2002. ARAA 40:539-577

[290] Rozo E, Nagai D, Keeton C, Kravtsov A. 2008. ApJ 687:22-38

[291] Rozo E, Wechsler RH, Rykoff ES, et al. 2010. ApJ 708:645-660

[292] Rudd DH, Zentner AR, Kravtsov AV. 2008. ApJ 672:19-32

[293] Ryden BS, Gunn JE. 1987. ApJ 318:15-31

[294] Salopek DS, Bond JR. 1990. Phys. Rev. D 42:3936-3962

[295] Sand DJ, Treu T, Ellis RS, Smith GP, Kneib JP. 2008. ApJ 674:711-727

[296] Sand DJ, Treu T, Smith GP, Ellis RS. 2004. ApJ 604:88-107 
REFERENCES

[297] Sarazin CL. 1986. Reviews of Modern Physics 58:1-115

[298] Sartoris B, Borgani S, Fedeli C, Matarrese S, Moscardini L, et al. 2010. MNRAS 407:2339-2354

[299] Schäfer BM, Koyama K. 2008. MNRAS 385:411-422

[300] Scherrer RJ, Weinberg DH. 1998. ApJ 504:607-611

[301] Schmidt F. 2009. Phys. Rev. D 80:123003

[302] Schmidt F, Hu W, Lima M. 2010. Phys. Rev. D 81:063005

[303] Schmidt F, Lima M, Oyaizu H, Hu W. 2009. Phys. Rev. D 79:083518

[304] Schmidt F, Vikhlinin A, Hu W. 2009. Phys. Rev. D 80:083505

[305] Schmidt RW, Allen SW. 2007. MNRAS 379:209-221

[306] Schuecker P, Finoguenov A, Miniati F, Böhringer H, Briel UG. 2004. AEFA 426:387-397

[307] Scoccimarro R, Sefusatti E, Zaldarriaga M. 2004. Phys. Rev. D 69:103513

[308] Sefusatti E, Vale C, Kadota K, Frieman J. 2007. ApJ 658:669-679

[309] Seljak U. 2000. MNRAS 318:203-213

[310] Sereno M, Zitrin A. 2012. MNRAS 419:3280-3291

[311] Shandera S, Dalal N, Huterer D. 2011. JCAP 3:17

[312] Shang C, Crotts A, Haiman Z. 2007. ApJ 671:136-145

[313] Sharma P, McCourt M, Quataert E, Parrish IJ. 2012. MNRAS :2294

[314] Sheth RK, Mo HJ, Tormen G. 2001. MNRAS 323:1-12

[315] Sheth RK, Tormen G. 1999. MNRAS 308:119-126

[316] Sheth RK, Tormen G. 2002. MNRAS 329:61-75

[317] Sijacki D, Pfrommer C, Springel V, Enßlin TA. 2008. MNRAS 387:1403-1415

[318] Sijacki D, Springel V, di Matteo T, Hernquist L. 2007. MNRAS 380:877-900

[319] Silvestri A, Trodden M. 2009. Reports on Progress in Physics 72:096901

[320] Simionescu A, Allen SW, Mantz A, Werner N, Takei Y, et al. 2011. Science 331:1576-

[321] Skillman SW, O'Shea BW, Hallman EJ, Burns JO, Norman ML. 2008. ApJ 689:10631077

[322] Slosar A, Hirata C, Seljak U, Ho S, Padmanabhan N. 2008. JCAP 8:31

[323] Smith S. 1936. ApJ 83:23-30

[324] Sotiriou TP, Faraoni V. 2010. Reviews of Modern Physics 82:451-497

[325] Springel V. 2005. MNRAS 364:1105-1134

[326] Springel V, Di Matteo T, Hernquist L. 2005. MNRAS 361:776-794

[327] Stanek R, Rasia E, Evrard AE, Pearce F, Gazzola L. 2010. ApJ 715:1508-1523

[328] Stanek R, Rudd D, Evrard AE. 2009. MNRAS 394:L11-L15

[329] Starobinsky AA. 1982. Physics Letters B 117:175-178

[330] Sun M. 2012. New Journal of Physics 14:045004

[331] Sun M, Sehgal N, Voit GM, Donahue M, Jones C, et al. 2011. ApJ 727:L49

[332] Sun M, Voit GM, Donahue M, Jones C, Forman W, Vikhlinin A. 2009. ApJ 693:11421172

[333] Sunyaev RA, Zeldovich IB. 1980. ARAA 18:537-560

[334] Sunyaev RA, Zeldovich YB. 1970. Comments on Astrophysics and Space Physics 2:6673

[335] Sunyaev RA, Zeldovich YB. 1972a. AEAA 20:189-200

[336] Sunyaev RA, Zeldovich YB. 1972b. Comments on Astrophysics and Space Physics 4:173-178

[337] Syer D, White SDM. 1998. MNRAS 293:337

[338] Taruya A, Koyama K, Matsubara T. 2008. Phys. Rev. D 78:123534

[339] Taylor JE, Navarro JF. 2001. ApJ 563:483-488

[340] Tinker J, Kravtsov AV, Klypin A, Abazajian K, Warren M, et al. 2008. ApJ 688:709-728

[341] Tinker JL, Robertson BE, Kravtsov AV, Klypin A, Warren MS, et al. 2010. ApJ 724:878- 
886

[342] Tissera PB, Dominguez-Tenreiro R. 1998. MNRAS 297:177-194

[343] Tissera PB, White SDM, Pedrosa S, Scannapieco C. 2010. MNRAS 406:922-935

[344] Tormen G. 1998. MNRAS 297:648-656

[345] Tornatore L, Borgani S, Springel V, Matteucci F, Menci N, Murante G. 2003. MNRAS 342:1025-1040

[346] Tozzi P, Norman C. 2001. ApJ 546:63-84

[347] Tyson JA, Kochanski GP, dell' Antonio IP. 1998. ApJ 498:L107

[348] Umetsu K, Broadhurst T, Zitrin A, Medezinski E, Coe D, Postman M. 2011a. ApJ 738:41

[349] Umetsu K, Broadhurst T, Zitrin A, Medezinski E, Hsu LY. 2011b. ApJ 729:127-143

[350] Valluri M, Vass IM, Kazantzidis S, Kravtsov AV, Bohn CL. 2007. ApJ 658:731-747

[351] van Albada GB. 1960. BAIN 15:165

[352] van Albada GB. 1961. AJ 66:590

[353] van Daalen MP, Schaye J, Booth CM, Dalla Vecchia C. 2011. MNRAS 415:3649-3665

[354] Vazza F, Brüggen M, Gheller C, Brunetti G. 2012. MNRAS 421:3375-3398

[355] Vazza F, Brunetti G, Kritsuk A, Wagner R, Gheller C, Norman M. 2009. AEA 504:33-43

[356] Venturi T, Giacintucci S, Dallacasa D, Cassano R, Brunetti G, et al. 2008. AEFA 484:327340

[357] Vikhlinin A. 2006. ApJ 640:710-715

[358] Vikhlinin A, Burenin RA, Ebeling H, Forman WR, Hornstrup A, et al. 2009a. ApJ 692:1033-1059

[359] Vikhlinin A, Burenin RA, Ebeling H, Forman WR, Hornstrup A, et al. 2009b. ApJ 692:1033-1059

[360] Vikhlinin A, Forman W, Jones C. 1999. ApJ 525:47-57

[361] Vikhlinin A, Kravtsov A, Forman W, Jones C, Markevitch M, et al. 2006. ApJ 640:691709

[362] Vikhlinin A, Kravtsov AV, Burenin RA, Ebeling H, Forman WR, et al. 2009c. ApJ 692:1060-1074

[363] Vikhlinin A, Murray S, Gilli R, Tozzi P, Paolillo M, et al. 2009d. In astro2010: The Astronomy and Astrophysics Decadal Survey, vol. 2010 of Astronomy

[364] Vitvitska M, Klypin AA, Kravtsov AV, Wechsler RH, Primack JR, Bullock JS. 2002. ApJ 581:799-809

[365] Vogt C, Enßlin TA. 2005. AEA 434:67-76

[366] Voit GM. 2005. Reviews of Modern Physics 77:207-258

[367] Voit GM. 2011. ApJ 740:28

[368] Voit GM, Balogh ML, Bower RG, Lacey CG, Bryan GL. 2003. ApJ 593:272-290

[369] Voit GM, Bryan GL. 2001. Nature 414:425-427

[370] Wagner C, Verde L. 2012. JCAP 3:2

[371] Wang J, White SDM. 2009. MNRAS 396:709-717

[372] Warren MS, Abazajian K, Holz DE, Teodoro L. 2006. ApJ 646:881-885

[373] Warren MS, Quinn PJ, Salmon JK, Zurek WH. 1992. ApJ 399:405-425

[374] Wechsler RH, Bullock JS, Primack JR, Kravtsov AV, Dekel A. 2002. ApJ 568:52-70

[375] Weinberg DH, Mortonson M, Einsenstein D, Hirata C, Riess A, Rozo E. 2012. Phys. Reports in press (arXiv/1201.2434)

[376] Werner N, Durret F, Ohashi T, Schindler S, Wiersma RPC. 2008. Sp. Sc. Rev. 134:337-362

[377] West MJ, Blakeslee JP. 2000. ApJ 543:L27-L30

[378] White M. 2001. AE्FA 367:27-32

[379] White M. 2002. ApJS 143:241-255

[380] White SDM. 1976. MNRAS 177:717-733

[381] White SDM, Rees MJ. 1978. MNRAS 183:341-358 
[382] Wojtak R, Łokas EL. 2010. MNRAS 408:2442-2456

[383] Wu HY, Zentner AR, Wechsler RH. 2010. ApJ 713:856-864

[384] Wu KKS, Fabian AC, Nulsen PEJ. 2000. MNRAS 318:889-912

[385] Younger JD, Bryan GL. 2007. ApJ 666:647-657

[386] Zeldovich YB, Klypin AA, Khlopov MY, Chechetkin VM. 1980. Soviet J. Nucl. Phys. 31:664

[387] Zentner AR. 2007. International Journal of Modern Physics D 16:763-815

[388] Zhao DH, Jing YP, Mo HJ, Börner G. 2009. ApJ 707:354-369

[389] Zhao DH, Mo HJ, Jing YP, Börner G. 2003. MNRAS 339:12-24

[390] Zhao GB, Li B, Koyama K. 2011. Phys. Rev. D 83:044007

[391] Zhuravleva IV, Churazov EM, Sazonov SY, Sunyaev RA, Forman W, Dolag K. 2010. MNRAS 403:129-150

[392] Zitrin A, Broadhurst T, Coe D, et al. 2011. ApJ 742:117

[393] Zwicky F. 1933. Helvetica Physica Acta 6:110-127

[394] Zwicky F. 1937. ApJ 86:217-246 